\title{
Structure of Chern-Simons scattering amplitudes from topological equivalence theorem and double-copy
}

\author{
Yan-Feng Hang, ${ }^{a}$ Hong-Jian He $\mathrm{He}^{a, b, c}$ and Cong Shen ${ }^{a}$ \\ ${ }^{a}$ Tsung-Dao Lee Institute 85 School of Physics and Astronomy, \\ Key Laboratory for Particle Astrophysics and Cosmology (MOE), \\ Shanghai Key Laboratory for Particle Physics and Cosmology, \\ Shanghai Jiao Tong University, Shanghai 200240, China \\ ${ }^{b}$ Institute of Modern Physics and Department of Physics, \\ Tsinghua University, Beijing 100084, China \\ ${ }^{c}$ Center for High Energy Physics, Peking University, \\ Beijing 100871, China \\ E-mail: yfhang@sjtu.edu.cn, hjhe@sjtu.edu.cn, cong.shen@epfl.ch
}

ABSTRACT: We study the mechanism of topological mass-generation for 3d Chern-Simons (CS) gauge theories, where the CS term can retain the gauge symmetry and make gauge boson topologically massive. Without CS term the $3 \mathrm{~d}$ massless gauge boson has a single physical transverse polarization state, while adding the CS term converts it into a massive physical polarization state and conserves the total physical degrees of freedom. We formulate the mechanism of topological mass-generation at $S$-matrix level. For this, we propose and prove a Topological Equivalence Theorem (TET) which connects the $N$-point scattering amplitude of the gauge boson's physical polarization states $\left(A_{\mathrm{P}}^{a}\right)$ to that of the transverse polarization states $\left(A_{\mathrm{T}}^{a}\right)$ under high energy expansion. We present a general $3 \mathrm{~d}$ power counting method on the leading energy dependence of the scattering amplitudes in both topologically massive Yang-Mills (TMYM) and topologically massive gravity (TMG) theories. With these, we uncover a general energy cancellation mechanism for $N$-gauge boson scattering amplitudes which predicts the cancellation $E^{4} \rightarrow E^{4-N}$ at tree level. Then, we compute the 4-gauge boson amplitudes of $A_{\mathrm{P}}^{a}$-states and $A_{\mathrm{T}}^{a}$-states, with which we explicitly demonstrate the TET and establish such energy cancellations for $N=4$. We further extend the double-copy approach to reconstruct the massive 4-graviton amplitude of TMG from the massive 4-gauge boson amplitude of TMYM. With these, we uncover striking large energy cancellations in the 4-graviton amplitude: $E^{12} \rightarrow E^{1}$, and establish its correspondence to the leading energy cancellation $E^{4} \rightarrow E^{0}$ in the 4-gauge boson amplitude of TMYM.

Keywords: Chern-Simons Theories, Field Theories in Lower Dimensions, Scattering Amplitudes, Topological Field Theories

ArXiv EPrint: 2110.05399 


\section{Contents}

1 Introduction 1

2 Topological mass generation in Chern-Simons gauge theories 4

3 Topological equivalence theorem for Chern-Simons gauge theories $\quad 7$

$\begin{array}{ll}3.1 \text { Topological equivalence theorem for topological mass generation } & 7\end{array}$

3.2 Power counting method for 3d Chern-Simons theories 9

3.3 Energy cancellations for topological scattering amplitudes 11

4 Topological scattering amplitudes and energy cancellations $\quad 12$

4.1 Topologically massive QED and scattering amplitudes 12

4.1.1 Scattering amplitudes of topologically massive scalar QED 13

$\begin{array}{lll}\text { 4.1.2 Scattering amplitudes of topologically massive spinor QED } & 15\end{array}$

$\begin{array}{lll}4.2 & \text { Topologically massive QCD and scattering amplitudes } & 17\end{array}$

$\begin{array}{lll}\text { 4.2.1 Scattering amplitudes of quark-antiquark annihilation } & 17\end{array}$

$\begin{array}{ll}\text { 4.2.2 Pure gauge boson scattering amplitudes } & 19\end{array}$

4.3 Unitarity bounds on TMYM and TMG theories 22

5 Structure of topological graviton amplitude from double-copy 23

$\begin{array}{ll}5.13 \text { d topologically massive gravity } & 24\end{array}$

5.2 Double-copy construction of graviton amplitude and energy cancellations $\quad 25$

6 Conclusions $\quad 30$

A Kinematics and Feynman rules of 3d CS gauge theories 33

B General power counting method in $d$-dimensions $\quad 34$

$\begin{array}{ll}\text { C Dirac spinors in }(2+1) d \text { spacetime } & 35\end{array}$

D Topological scattering amplitudes with matter fields $\quad 36$

$\begin{array}{lll}\text { E Graviton propagator and scattering amplitude in TMG } & 37\end{array}$

\section{Introduction}

Chern-Simons gauge theories in (2+1)-dimensional (3d) spacetime play an important role in studying modern quantum field theories for particle physics and condensed matter physics [1-4]. Such 3d theories can always contain gauge-invariant mass terms of gauge 
bosons through the topological mass-generation à la Chern-Simons (CS) [5], without invoking the conventional Higgs mechanism $[6-10]^{1}$ in the $4 \mathrm{~d}$ standard model (SM).

In this work, we study the dynamics of $3 \mathrm{~d}$ topological mass-generation for the (Abelian and non-Abelian) gauge bosons $A_{\mu}^{a}$. A spin-1 massless gauge boson in $3 \mathrm{~d}$ contains only one physical degree of freedom (DoF) which is the transversely polarized state $A_{\mathrm{T}}^{a}$. Including the gauge-invariant topological CS term converts this massless transverse polarization state $A_{\mathrm{T}}^{a}$ into a massive physical state $A_{\mathrm{P}}^{a}$. We formulate this $3 \mathrm{~d}$ topological mass-generation mechanism at $S$-matrix level. For this, we propose and prove a Topological Equivalence Theorem (TET) which connects the $N$-point scattering amplitudes of the physical polarization states of massive gauge bosons $\left(A_{\mathrm{P}}^{a}\right)$ to the scattering amplitudes of the corresponding transversely polarized gauge boson states $\left(A_{\mathrm{T}}^{a}\right)$ under high energy expansion. This differs essentially from the conventional equivalence theorem (ET) (for a comprehensive review of the 4-dimensional ET, [12]) in the 4d SM because the 3d gauge bosons acquire gaugeinvariant topological mass-term without invoking the conventional Higgs mechanism [6-10]. We note that the Kaluza-Klein ET for compactified gauge theories (KK GAET) [13-16] was formulated for the compactified 5d Yang-Mills theories which realize a geometric Higgs mechanism with the 5th component of 5 d gauge field converted to the longitudinal component of the corresponding $4 \mathrm{~d}$ massive KK gauge boson. But our TET also has essential difference from the KK GAET, because the 5d gauge symmetry is spontaneously broken by compactification down to the $4 \mathrm{~d}$ residual gauge symmetry of the massless zero-modes and the induced KK gauge boson mass-term is not gauge-invariant. In contrast, the $3 \mathrm{~d}$ CS term for the topological mass-generation of gauge bosons can be manifestly gauge-invariant and the $3 \mathrm{~d}$ gauge symmetry is unchanged before and after including the CS term.

We present a general $3 \mathrm{~d}$ power counting method to count the leading energy-power dependence of the scattering amplitudes in both topologically massive Yang-Mills (TMYM) theory and topologically massive gravity (TMG) theory. Using the TET and power counting method for the $3 \mathrm{~d}$ TMYM theory, we uncover that despite the individual diagrams in a given $N$-particle scattering amplitude $(N \geqslant 4)$ of on-shell physical gauge bosons having leading energy dependence of $E^{4}$ at tree level, they have to cancel down to $E^{4-N}$ in the full tree-level amplitude. We will demonstrate that the TET provides a general theoretical mechanism to guarantee such nontrivial energy cancellations: $E^{4} \rightarrow E^{4-N}$, without invoking any conventional Higgs boson. For the massive 4-gauge boson scattering amplitudes at tree level, we will prove explicitly the large energy cancellations of $E^{4} \rightarrow E^{0}$ under high energy expansion.

Furthermore, using the scattering amplitude of topologically massive gauge bosons in the 3d TMYM theory, we will reconstruct the topologically massive graviton scattering amplitude of the 3d TMG theory, by extending the conventional double-copy method of Bern-Carrasco-Johansson (BCJ) [17-19] for massless gauge/gravity theories to the current 3d topologically massive gauge/gravity theories. The BCJ method was inspired by the Kawai-Lewellen-Tye (KLT) [20] relation which connects the product of open string ampli-

\footnotetext{
${ }^{1}$ The conventional Higgs mechanism [6-10] is sometimes also called Brout-Englert-Higgs (BEH) mechanism or Anderson-Higgs mechanismin the literature (cf. [11], its part IV.6).
} 
tudes to that of the closed string at tree level. Analyzing the properties of the heterotic string and open string amplitudes can prove and refine parts of the BCJ conjecture [21]. Many studies appeared in the literature to test the double-copy conjecture in massless gauge/gravity field theories [19], and some recent works attempted to extend the doublecopy method to the $4 \mathrm{~d}$ massive YM theory versus Fierz-Pauli-like massive gravity [22-25], to the KK-inspired effective gauge theory with extra global U(1) [26], and to the compactified $5 \mathrm{~d}$ KK gauge/gravity theories and KK string theories [27, 28]. Double-copies of threeand two-algebra gauge theories were considered previously for the $3 \mathrm{~d}$ supersymmetric theories [29-31], and some double-copy analyses for the amplitudes with matter fields in $3 \mathrm{~d}$ CS gauge theory as well as the study of $3 \mathrm{~d}$ covariant color-kinematics duality appeared very recently $[32-34]$.

We stress that the topological mass-generation for gauge bosons and gravitons in the 3d TMYM and TMG theories can be realized in a fully gauge-invariant way under the path integral formulation, which is important for the successful double-copy construction in the massive gauge/gravity theories. In this work, we will use an extended double-copy approach to reconstruct the massive 4-graviton amplitude of the TMG theory from the corresponding massive 4-gauge boson amplitude of the TMYM theory with properly improved kinematic numerators. Our findings demonstrate a series of strikingly large energy cancellations, $E^{12} \rightarrow E^{1}$, in the massive 4-graviton amplitude under high energy expansion. With these we establish a new correspondence between the two types of leading energy cancellations in the massive scattering amplitudes: $E^{4} \rightarrow E^{0}$ in the TMYM theory and $E^{12} \rightarrow E^{1}$ in the $T M G$ theory.

This paper is organized as follows. In section 2, we study the mechanism of the topological mass-generation in the $3 \mathrm{~d}$ CS gauge theories at the Lagrangian level via path integral formulation. We identify the conversion of transverse polarization state $A_{\mathrm{T}}^{a}$ in the massless theory into the massive physical polarization state $A_{\mathrm{P}}^{a}$ under the topological mass-generation of the CS gauge theories. In section 3.1, we propose and prove the TET which connects the $N$-point $A_{\mathrm{P}}^{a}$-amplitudes to the corresponding $A_{\mathrm{T}}^{a}$-amplitudes under high energy expansion. Using the TET, we can formulate the mechanism of topological massgeneration at $S$-matrix level. Then, in section 3.2, we present the general $3 \mathrm{~d}$ power counting rules on the leading energy dependence of the scattering amplitudes in both the CS gauge theories and the TMG theory. Using the TET and the power counting rule, we prove in section 3.3 a general energy cancellation mechanism for the $N$-gauge boson scattering amplitudes which predicts the cancellation $E^{4} \rightarrow E^{4-N}$ at tree level. For sections 4.1-4.2, we first compute the 4-point matter-induced gauge boson amplitudes, and then compute the pure 4-gauge boson amplitudes for the $A_{\mathrm{P}}^{a}$-states and $A_{\mathrm{T}}^{a}$-states in the Abelian and non-Abelian CS gauge theories. These analyses explicitly demonstrate the TET for the case of $N=4$, and establish the energy cancellations $E^{2} \rightarrow E^{0}$ in the 4-point amplitudes with just two gauge bosons (either Abelian or non-Abelian CS theories) and the energy cancellations $E^{4} \rightarrow E^{0}$ in the 4-gauge boson amplitudes (TMYM theory). In section 4.3, we analyze the perturbative unitarity bounds for both the TMYM theory and the TMG theory. We demonstrate that their partial wave amplitudes can exhibit good high energy behaviors. In section 5, we further extend the double-copy approach to reconstruct the 
massive 4-graviton amplitude of the TMG from the massive 4-gauge boson amplitude of the TMYM. With these, we uncover strikingly large energy cancellations in the 4-graviton amplitude: $E^{12} \rightarrow E^{1}$, and establish its correspondence to the leading energy cancellation $E^{4} \rightarrow E^{0}$ in the massive 4-gauge boson amplitude of the TMYM. We conclude in section 6 . Finally, we provide more derivations and formulas in appendices $\mathrm{A}-\mathrm{E}$ which are used for the analyses in the main text.

\section{Topological mass generation in Chern-Simons gauge theories}

We consider the 3d topological massive gauge theories including the Chern-Simons (CS) Lagrangian with Abelian or non-Abelian gauge symmetry, where the former may be denoted as Topologically Massive QED (TMQED) and the latter as Topologically Massive Yang-Mills (TMYM) theory. In either case, the CS Lagrangian provides a gauge-invariant topological mass-term for the 3d gauge bosons. The 3d TMQED and TMYM Lagrangians have their gauge sectors take the following forms:

$$
\begin{aligned}
\mathcal{L}_{\mathrm{TMQED}} & =-\frac{1}{4} F_{\mu \nu}^{2}+\frac{1}{2} \widetilde{m} \varepsilon^{\mu \nu \rho} A_{\mu} \partial_{\nu} A_{\rho}, \\
\mathcal{L}_{\mathrm{TMYM}} & =-\frac{1}{2} \operatorname{tr} \mathbf{F}_{\mu \nu}^{2}+\widetilde{m} \varepsilon^{\mu \nu \rho} \operatorname{tr}\left(\mathbf{A}_{\mu} \partial_{\nu} \mathbf{A}_{\rho}-\frac{\mathrm{i} 2 g}{3} \mathbf{A}_{\mu} \mathbf{A}_{\nu} \mathbf{A}_{\rho}\right),
\end{aligned}
$$

where the non-Abelian gauge field $\mathbf{A}_{\mu}=A_{\mu}^{a} T^{a}$, and its field strength $\mathbf{F}_{\mu \nu}=F_{\mu \nu}^{a} T^{a}$ with $\mathbf{F}_{\mu \nu}=\partial_{\mu} \mathbf{A}_{\nu}-\partial_{\nu} \mathbf{A}_{\mu}-\mathrm{i} g\left[\mathbf{A}_{\mu}, \mathbf{A}_{\nu}\right]$ and $T^{a}$ denotes the generator of the non-Abelian group $\mathrm{SU}(N)$. The gauge coupling $g$ has mass-dimension $\frac{1}{2}$. The gauge boson acquires a topological mass $m=|\widetilde{m}|$ from the CS term, and the ratio $\mathfrak{s}=\widetilde{m} / m= \pm 1$ corresponds to its spin projection [1-3]. The mass parameter $\widetilde{m}$ is related to the CS level $n=4 \pi \widetilde{m} / g^{2} \in \mathbb{Z}[3,4]$. The CS terms in eq. (2.1) violate the discrete symmetries $P, T$ and $C P$.

For the TMQED (2.1a), the action $\int \mathrm{d}^{3} x \mathcal{L}_{\text {TMQED }}$ is gauge-invariant up to a total derivative which vanishes at the boundary for trivial topology. For the TMYM theory (2.1b), under the gauge transformation $\mathbf{A}_{\mu} \rightarrow \mathbf{A}_{\mu}^{\prime}=U^{-1} \mathbf{A}_{\mu} U+\frac{\mathrm{i}}{g} U^{-1} \partial_{\mu} U$, the action changes by

$$
\begin{aligned}
\Delta S_{\mathrm{TMYM}} & =2 \pi n w+\int \mathrm{d}^{3} x\left[\mathrm{i} \tilde{m} g^{-1} \varepsilon^{\mu \nu \rho} \partial_{\nu} \operatorname{tr}\left(\partial_{\mu} U U^{-1} A_{\rho}\right)\right], \\
w & =\frac{1}{24 \pi^{2}} \int \mathrm{d}^{3} x\left[\varepsilon^{\mu \nu \rho} \operatorname{tr}\left(U^{-1} \partial_{\mu} U U^{-1} \partial_{\nu} U U^{-1} \partial_{\rho} U\right)\right],
\end{aligned}
$$

where $w$ is the winding number which follows from the homotopy group $\Pi_{3}[\mathrm{SU}(N)] \cong \mathbb{Z}[4]$. Hence, eq. (2.2a) will not contribute to the path integral since $e^{\mathrm{i} 2 \pi n w}=1$, and the second term is a total derivative (similar to the Abelian case).

With the path integral formulation, we can add the covariant gauge-fixing term and the Faddeev-Popov ghost term:

$$
\begin{aligned}
& \mathcal{L}_{\mathrm{GF}}=-\frac{1}{2 \xi}\left(\mathcal{F}^{a}\right)^{2}, \quad \mathcal{F}^{a}=\partial^{\mu} A_{\mu}^{a}, \\
& \mathcal{L}_{\mathrm{FP}}=\bar{c}^{a} \partial^{\mu}\left(\delta^{a b} \partial_{\mu}-g C^{a b c} A_{\mu}^{c}\right) c^{b},
\end{aligned}
$$


where $C^{a b c}$ is the gauge group structure constant and $\left(c^{a}, \bar{c}^{a}\right)$ denote the Faddeev-Popov ghost and anti-ghost fields. Eq. (2.3) can be reduced to the Abelian case by simply setting $C^{a b c}=0$ and $A_{\mu}^{a}=A_{\mu}$. So, hereafter we need not to specify the Abelian case unless needed. The quantized CS action $\int \mathrm{d}^{3} x\left(\mathcal{L}+\mathcal{L}_{\mathrm{GF}}+\mathcal{L}_{\mathrm{FP}}\right)$ is BRST-invariant (Becchi-Rouet-StoraTyutin), with which we can derive the relevant BRST identities.

The equation of motion (EOM) for the massive gauge boson $A_{\mu}^{a}$ can be derived from the quadratic part of the CS action,

$$
\left[\eta^{\mu \nu} \partial^{2}+\left(\xi^{-1}-1\right) \partial^{\mu} \partial^{\nu}+\widetilde{m} \varepsilon^{\mu \rho \nu} \partial_{\rho}\right] A_{\nu}^{a}=0
$$

which describes the propagation of the free field $A_{\mu}^{a}$. For the on-shell wave solution $A_{\mu}^{a} \sim$ $\epsilon_{\mu}(p) e^{-\mathrm{i} p \cdot x}$ with $p^{\mu} A_{\mu}^{a}=0$, the polarization vector should satisfy the equation

$$
\left(m \eta^{\mu \nu}-\mathfrak{i} \mathfrak{s} \varepsilon^{\mu \rho \nu} p_{\rho}\right) \epsilon_{\nu}(p)=0,
$$

under the on-shell condition $p^{2}=-m^{2}$ and with $\mathfrak{s}=\widetilde{m} / m= \pm 1$. The 3d Poincaré group $\operatorname{ISO}(2,1)$ contains the proper Lorentz group $\mathrm{SO}(2,1)$ and translations. The little group is $\mathbb{Z}_{2} \otimes \mathbb{R}$ for massless particles and $\mathrm{SO}(2)$ for massive particles [35]. The Poincaré algebra is characterized by two Casimir operators $\left(P^{2}, W\right)=\left(P_{\mu} P^{\mu}, P_{\mu} J^{\mu}\right)$, where $W$ is the PauliLubanski pseudoscalar and the angular momentum $J^{\mu}$ can be generally expressed as [36]:

$$
J^{\mu}=-\mathrm{i} \varepsilon^{\mu \nu \alpha} p_{\nu} \frac{\partial}{\partial p_{\alpha}}-\mathfrak{s} \frac{p^{\mu}+\eta^{\mu} m}{p \cdot \eta-m}
$$

with $\eta^{\mu}=(1,0,0)$. Thus, in the rest frame it gives $W=P \cdot J=-\mathfrak{s} m$. We see that the spin is a pseudoscalar and takes the values $\mathfrak{s}= \pm 1$ for gauge fields $A_{\mu}^{a}$. The polarization state with either $\mathfrak{s}=+1$ or $\mathfrak{s}=-1$ is physically equivalent. (More discussions on the gauge boson polarization vector are given in appendix A.)

Note that the $3 \mathrm{~d}$ massless gauge field can be viewed as a scalar field of spin-0 with one physical degree of freedom (DoF) [35, 36]. Including the CS term does not add any new field, and the total physical DoF remains as one because the physical DoF of $A_{\mu}^{a}$ should be conserved [36, 37]. For the on-shell one-particle state, the $3 \mathrm{~d}$ massless gauge boson has a single (transverse) physical polarization state $A_{\mathrm{T}}^{a}=\epsilon_{\mathrm{T}}^{\mu} A_{\mu}^{a}$. As the physical DoF is conserved, the CS term could only convert the massless $A_{\mathrm{T}}^{a}$ state into a massive physical polarization state $A_{\mathrm{P}}^{a}=\epsilon_{\mathrm{P}}^{\mu} A_{\mu}^{a}$.

For the on-shell gauge boson in the rest frame with momentum $p^{\mu}=(m, 0,0) \equiv \bar{p}^{\mu}$, the physical polarization vector $\epsilon_{\mathrm{P}}^{\mu}(\bar{p})$ can be solved from eq. (2.5):

$$
\epsilon_{\mathrm{P}}^{\mu}(\bar{p})=\frac{1}{\sqrt{2}}(0,1,-\mathrm{i} \mathfrak{s}),
$$

in agreement with [37, 38]. Then, by making a Lorentz boost we can express $\epsilon_{\mathrm{P}}^{\mu}(p)$ in the moving frame:

$$
\epsilon_{\mathrm{P}}^{\mu}(p)=\frac{1}{\sqrt{2}}\left(\frac{\mathrm{i} p_{1}+\mathfrak{s} p_{2}}{m}, \mathrm{i}+\frac{p_{1}\left(\mathrm{i} p_{1}+\mathfrak{s} p_{2}\right)}{m\left(m-p_{0}\right)}, \mathfrak{s}+\frac{p_{2}\left(\mathrm{i} p_{1}+\mathfrak{s} p_{2}\right)}{m\left(m-p_{0}\right)}\right),
$$


which agrees with [38] up to an overall factor i. The on-shell physical polarization vector $\epsilon_{\mathrm{P}}^{\mu}(p)$ obeys the conditions $\epsilon_{\mathrm{P}}^{\mu} \epsilon_{\mathrm{P} \mu}^{*}=1$ and $p_{\mu} \epsilon_{P}^{\mu}=0$. We can express the general momentum $p^{\mu}$ in a familiar form $p^{\mu}=E\left(1, \beta s_{\theta}, \beta c_{\theta}\right)$, where the notations $p^{0}=-p_{0}=E,\left(s_{\theta}, c_{\theta}\right)=$ $(\sin \theta, \cos \theta), \beta=\sqrt{1-m^{2} / E^{2}}$, and $\theta$ denotes the angle between the moving direction and $y$-axis. With these, we can rewrite the polarization vector (2.8) as follows:

$$
\epsilon_{\mathrm{P}}^{\mu}(p)=\frac{1}{\sqrt{2}}\left(\bar{E} \beta, \bar{E} s_{\theta}+\mathrm{i} \mathfrak{s} c_{\theta}, \bar{E} c_{\theta}-\mathrm{i} \mathfrak{s} s_{\theta}\right),
$$

where $\bar{E}=E / m$ and we have removed an irrelevant overall phase factor. Inspecting the structure of the physical polarization vector (2.9), we derive the following general decomposition:

$$
\epsilon_{\mathrm{P}}^{\mu}=\frac{1}{\sqrt{2}}\left(\epsilon_{\mathrm{T}}^{\mu}+\epsilon_{\mathrm{L}}^{\mu}\right),
$$

which contains the transverse and longitudinal polarization vectors $\left(\epsilon_{\mathrm{T}}^{\mu}, \epsilon_{\mathrm{L}}^{\mu}\right)$ of the massive gauge boson $A_{\mu}^{a}$,

$$
\epsilon_{\mathrm{T}}^{\mu}=\left(0, \mathrm{i} \mathfrak{s} c_{\theta},-\mathrm{i} \mathfrak{s} s_{\theta}\right), \quad \epsilon_{\mathrm{L}}^{\mu}=\bar{E}\left(\beta, s_{\theta}, c_{\theta}\right) .
$$

Thus, we have the relation for the on-shell polarization states of $A_{\mu}^{a}$ :

$$
A_{\mathrm{P}}^{a}=\frac{1}{\sqrt{2}}\left(A_{\mathrm{T}}^{a}+A_{\mathrm{L}}^{a}\right),
$$

where $\left(A_{\mathrm{P}}^{a}, A_{\mathrm{T}}^{a}, A_{\mathrm{L}}^{a}\right)=\left(\epsilon_{\mathrm{P}}^{\mu}, \epsilon_{\mathrm{T}}^{\mu}, \epsilon_{\mathrm{L}}^{\mu}\right) A_{\mu}^{a}$. The gauge boson $A_{\mu}^{a}$ also has an unphysical scalar polarization state $A_{\mathrm{S}}^{a}=\epsilon_{\mathrm{S}}^{\mu} A_{\mu}^{a}$ with $\epsilon_{\mathrm{S}}^{\mu}=p^{\mu} / \mathrm{m}$. It is important to note that the polarization vectors $\left(\epsilon_{\mathrm{P}}^{\mu}, \epsilon_{\mathrm{L}}^{\mu}, \epsilon_{\mathrm{S}}^{\mu}\right)$ are all enhanced by energy and scale as $\mathcal{O}(E / m)$ under the high energy expansion. The $3 \mathrm{~d}$ gauge boson $A_{\mu}^{a}$ has 3 possible polarization states in total, including 1 physical polarization and 2 unphysical polarizations. In the massless case $(m=0), A_{\mu}^{a}$ contains 1 physical transverse polarization state $A_{\mathrm{T}}^{a}=\epsilon_{\mathrm{T}}^{\mu} A_{\mu}^{a}$ and 2 unphysical (longitudinal,scalar) polarization states $\left(A_{\mathrm{L}}^{a}, A_{\mathrm{S}}^{a}\right)=\left(\epsilon_{\mathrm{L}}^{\mu} A_{\mu}^{a}, \epsilon_{\mathrm{S}}^{\mu} A_{\mu}^{a}\right)$ with $\epsilon_{\mathrm{L}}^{\mu}+\epsilon_{\mathrm{S}}^{\mu} \propto p^{\mu}$; while for the massive case with $\mathrm{CS}$ term $(m \neq 0), A_{\mu}^{a}$ includes 1 physical polarization state $A_{\mathrm{P}}^{a}$ as in eq. (2.12) and 2 orthogonal unphysical polarization states

$$
\begin{aligned}
& A_{\mathrm{X}}^{a}=\epsilon_{\mathrm{X}}^{\mu} A_{\mu}^{a}=\frac{1}{\sqrt{2}}\left(A_{\mathrm{T}}^{a}-A_{\mathrm{L}}^{a}\right), \\
& A_{\mathrm{S}}^{a}=\epsilon_{\mathrm{S}}^{\mu} A_{\mu}^{a},
\end{aligned}
$$

where $\epsilon_{\mathrm{X}}^{\mu}=\left(\epsilon_{\mathrm{T}}^{\mu}-\epsilon_{\mathrm{L}}^{\mu}\right) / \sqrt{2}$ and $\epsilon_{\mathrm{S}}^{\mu}=p^{\mu} / m$ which obey the orthogonal conditions $\epsilon_{\mathrm{P}} \cdot \epsilon_{\mathrm{X}}^{*}=$ $\epsilon_{\mathrm{P}} \cdot \epsilon_{\mathrm{S}}^{*}=\epsilon_{\mathrm{X}} \cdot \epsilon_{\mathrm{S}}^{*}=0$. We see that adding the CS term for gauge boson $A_{\mu}^{a}$ dynamically generates a new physical polarization state $A_{\mathrm{P}}^{a}$ of spin- 1 (which has mass $m$ and is composed of $\left.A_{\mathrm{T}}^{a}+A_{\mathrm{L}}^{a}\right)$, and converts its orthogonal combination $A_{\mathrm{X}}^{a} \propto\left(A_{\mathrm{T}}^{a}-A_{\mathrm{L}}^{a}\right)$ into the unphysical state, while the scalar-polarization state $A_{\mathrm{S}}^{a}=\epsilon_{\mathrm{S}}^{\mu} A_{\mu}^{a}$ (with $\epsilon_{\mathrm{S}}^{\mu} \propto p^{\mu}$ ) remains unphysical as constrained by the gauge-fixing function $\mathcal{F}^{a}=-\mathrm{i} p^{\mu} A_{\mu}^{a}$ in eq. (2.3a).

The above mechanism of $3 \mathrm{~d}$ topological mass-generation might be called a "topological Higgs mechanism" to resemble the dynamical conversion of $\left(A_{\mathrm{T}}^{a}+A_{\mathrm{L}}^{a}\right)$ into the massive physical state $A_{\mathrm{P}}^{a}$ of the gauge field $A_{\mu}^{a}$, while making the orthogonal combination 
$A_{\mathrm{X}}^{a} \propto\left(A_{\mathrm{T}}^{a}-A_{\mathrm{L}}^{a}\right)$ be an unphysical "Goldstone boson" state. However, we stress that this mechanism of topological mass-generation of gauge bosons differs from the conventional Higgs mechanism [6-10] in essential ways: (i)topological CS mass-term automatically holds the exact gauge symmetry in the path integral formulation, without invoking any spontaneous gauge symmetry breaking by the vacuum of Higgs potential; (ii)before including the CS term, the transverse $A_{\mathrm{T}}^{a}$ is the physical polarization state and is exactly massless as ensured by the gauge symmetry; while after including the CS term, $A_{\mathrm{T}}^{a}$ combines with $A_{\mathrm{L}}^{a}$ to form the massive physical state $A_{\mathrm{P}}^{a}$ and makes its orthogonal combination $A_{\mathrm{X}}^{a}$ become unphysical; hence there is no spontaneous symmetry breaking invoked to generate massless Goldstone boson, nor is there any extra physical Higgs boson component; (iii)the massive physical gauge boson state $A_{\mathrm{P}}^{a}$ is converted from the massless transverse polarization state $A_{\mathrm{T}}^{a}$ combined with the longitudinal polarization state $A_{\mathrm{L}}^{a}$ via eqs. (2.12) and (2.13a). The single physical degree of freedom of $A_{\mu}^{a}$ is conserved before and after adding the CS term, through the topological conversion $A_{\mathrm{T}}^{a} \rightarrow A_{\mathrm{P}}^{a}$. Taking the massless limit $m \rightarrow 0$, we see that the massive state $A_{\mathrm{P}}^{a}$ disappears and the massless state $A_{\mathrm{T}}^{a}$ is released to be the physical transverse polarization, while the longitudinal state $A_{\mathrm{L}}^{a}$ becomes fully unphysical again. As we will demonstrate shortly, in the high energy limit the scattering amplitudes of the physical polarization states $\left(A_{\mathrm{P}}^{a}\right)$ equal the corresponding amplitudes of the transverse polarization states $\left(A_{\mathrm{T}}^{a}\right)$, which means that the $A_{\mathrm{P}}^{a}$ state remembers its origin of $A_{\mathrm{T}}^{a}$ state under the limit $m / E \rightarrow 0$.

\section{Topological equivalence theorem for Chern-Simons gauge theories}

As shown above, for the $3 \mathrm{~d}$ topological gauge theories (2.1), the Chern-Simons (CS) Lagrangian generates a topological mass for gauge boson $A_{\mu}^{a}$ by converting the massless transverse polarization state $A_{\mathrm{T}}^{a}$ (combined with the longitudinal polarization state $A_{\mathrm{L}}^{a}$ ) into the massive physical polarization state $A_{\mathrm{P}}^{a}$. We will formulate this mechanism of topological mass-generation at the $S$-matrix level by proposing a Topological Equivalence Theorem (TET), which quantitatively connects the scattering amplitudes of $A_{\mathrm{P}}^{a}$ 's to the corresponding amplitudes of the $A_{\mathrm{T}}^{a}$ 's in the high energy limit $m / E \rightarrow 0$.

\subsection{Topological equivalence theorem for topological mass generation}

Inspecting the quantized CS Lagrangians (2.1) and (2.3) and following the method in refs. [12, 39, 40], we can derive the following Slavnov-Taylor-type identity in momentum space:

$$
\left\langle 0\left|\mathcal{F}^{a_{1}}\left(p_{1}\right) \mathcal{F}^{a_{2}}\left(p_{2}\right) \cdots \mathcal{F}^{a_{N}}\left(p_{N}\right) \Phi\right| 0\right\rangle=0,
$$

which is based on the $3 \mathrm{~d}$ gauge symmetry, where $\mathcal{F}^{a}$ is the gauge-fixing function defined in eq. (2.3a), and the symbol $\Phi$ denotes any other on-shell physical fields after the LSZ (Lehmann-Symanzik-Zimmermann) amputation. Since the function $\mathcal{F}^{a}$ contains only a single gauge field $A_{\mu}^{a}$ having no mixing with any other field, it is straightforward to amputate each external $\mathcal{F}^{a}$ line by the LSZ reduction. We impose the on-shell condition $p_{j}^{2}=-m^{2}$ for each external momentum. In the momentum space, we can express the 
gauge-fixing function $\mathcal{F}^{a}=-\mathrm{i} p^{\mu} A_{\mu}^{a}=-\mathrm{i} m A_{\mathrm{S}}^{a}$. We also deduce $v^{\mu} \equiv \epsilon_{\mathrm{L}}^{\mu}-\epsilon_{\mathrm{S}}^{\mu}=\mathcal{O}(m / E)$. With eq. (2.10), we can express the scalar polarization vector $\epsilon_{\mathrm{S}}^{\mu}$ as

$$
\epsilon_{\mathrm{S}}^{\mu}=\sqrt{2} \epsilon_{\mathrm{P}}^{\mu}-\left(\epsilon_{\mathrm{T}}^{\mu}+v^{\mu}\right) .
$$

Thus, we derive the following formula for the gauge-fixing function:

$$
\begin{aligned}
\mathcal{F}^{a} & =-\mathrm{i} \sqrt{2} m\left(A_{\mathrm{P}}^{a}-\Omega^{a}\right), \\
\Omega^{a} & =\frac{1}{\sqrt{2}}\left(A_{\mathrm{T}}^{a}+v^{a}\right),
\end{aligned}
$$

where $\left(A_{\mathrm{P}}^{a}, A_{\mathrm{T}}^{a}\right)=\left(\epsilon_{\mathrm{P}}^{\mu}, \epsilon_{\mathrm{T}}^{\mu}\right) A_{\mu}^{a}$ and $v^{a}=v^{\mu} A_{\mu}^{a}$ with $v^{\mu}=\epsilon_{\mathrm{L}}^{\mu}-\epsilon_{\mathrm{S}}^{\mu}=\mathcal{O}(m / E)$. With these and eq. (3.1) after the LSZ reduction, we can derive the following TET identity:

$$
\mathcal{T}\left[A_{\mathrm{P}}^{a_{1}}, \cdots, A_{\mathrm{P}}^{a_{N}}, \Phi\right]=\mathcal{T}\left[\Omega^{a_{1}}, \cdots, \Omega^{a_{N}}, \Phi\right],
$$

where we have made use of the fact that an amplitude including one or more external $\mathcal{F}$ lines plus any other external on-shell physical fields (such as $A_{\mathrm{P}}^{a}$ and/or $\Phi$ ) must vanish due to the identity (3.1). Thus, we can expand the TET identity as follows:

$$
\begin{aligned}
\mathcal{T}\left[A_{\mathrm{P}}^{a_{1}}, \cdots, A_{\mathrm{P}}^{a_{N}}, \Phi\right] & =\mathcal{T}\left[\tilde{A}_{\mathrm{T}}^{a_{1}}, \cdots, \tilde{A}_{\mathrm{T}}^{a_{N}}, \Phi\right]+\mathcal{T}_{v}, \\
\mathcal{T}_{v} & =\sum_{j=1}^{N} \mathcal{T}\left[\tilde{v}^{a_{1}}, \cdots, \tilde{v}^{a_{j}}, \tilde{A}_{\mathrm{T}}^{a_{j+1}}, \cdots, \tilde{A}_{\mathrm{T}}^{a_{N}}, \Phi\right],
\end{aligned}
$$

where for convenience we have adopted the notations $\tilde{A}_{\mathrm{T}}^{a}=\frac{1}{\sqrt{2}} A_{\mathrm{T}}^{a}$ and $\tilde{v}^{a}=\frac{1}{\sqrt{2}} v^{a}$. Under the high energy expansion, the residual term behaves as $\mathcal{T}_{v}=\mathcal{O}(m / E) \ll 1$ due to the suppression factor $v^{\mu}$. Thus, we can derive the Topological Equivalence Theorem (TET):

$$
\mathcal{T}\left[A_{\mathrm{P}}^{a_{1}}, \cdots, A_{\mathrm{P}}^{a_{N}}, \Phi\right]=\mathcal{T}\left[\tilde{A}_{\mathrm{T}}^{a_{1}}, \cdots, \tilde{A}_{\mathrm{T}}^{a_{N}}, \Phi\right]+\mathcal{O}\left(\frac{m}{E}\right),
$$

which states that the $A_{\mathrm{P}}^{a}$-amplitude equals the corresponding $A_{\mathrm{T}^{-}}^{a}$-amplitude in the high energy limit. We note that different from the conventional equivalence theorem (ET) [39$41]^{2}$ for the case of the SM Higgs mechanism, the right-hand-side (r.h.s.) of eq. (3.5) or eq. (3.6) receives no multiplicative modification factor at loop level. This is because in the present case both $A_{\mathrm{P}}^{a}$ and $A_{\mathrm{T}}^{a}$ belong to the same gauge field $A_{\mu}^{a}$ and the LSZ reduction on the left-hand-side (1.h.s.) of eq. (3.1) becomes much simpler.

Finally, we note that our present formulation of the TET (3.6) in the 3d CS gauge theories differs essentially from the conventional ET [12] in the 4d SM because the $3 \mathrm{~d}$ gauge bosons acquire gauge-invariant topological mass-term without invoking the conventional Higgs mechanism [6-10]. We also note that the KK GAET [13-16] for the compactified 5d Yang-Mills theories formulates the geometric Higgs mechanism at $S$-matrix level where the 5 th component of $5 \mathrm{~d}$ gauge field is converted to the longitudinal component of the

\footnotetext{
${ }^{2}$ The $4 \mathrm{~d}$ ET in the presence of the Higgs-gravity interactions was established in refs. [42, 43] which can be applied to studying cosmological models (such as the Higgs inflation [42-49]) or to testing self-interactions of weak gauge bosons and Higgs bosons [42, 43, 50].
} 
corresponding 4d massive KK gauge boson. But our TET has essential difference from the KK GAET because the $5 \mathrm{~d}$ gauge symmetry is spontaneously broken down to the $4 \mathrm{~d}$ residual gauge symmetry of zero-modes by the boundary conditions of compactification and the induced KK gauge boson mass-term is not gauge-invariant. On the contrary, the $3 \mathrm{~d}$ CS term for the topological mass-generation of gauge bosons can be manifestly gauge-invariant, and the inclusion of CS term does not change the $3 \mathrm{~d}$ gauge symmetry of the theory.

\subsection{Power counting method for 3d Chern-Simons theories}

In this subsection, we derive new energy power counting rules on the scattering amplitudes in the $3 \mathrm{~d}$ topologically massive gauge and gravity theories. We also present a general energy power counting rule on the $d$-dimensional scattering amplitudes in appendix B.

We note that Weinberg proposed a power counting method for the $4 \mathrm{~d}$ ungauged nonlinear $\sigma$-model as an effective theory of low energy QCD [51]. The extensions of Weinberg's power counting method to the compactified 5d Kaluza-Klein (KK) gauge theories and 5d KK gravity theory were recently given in ref. [27]. ${ }^{3}$ Weinberg's power counting method includes the following key points: (i).For an $S$-matrix element $\mathbb{S}$, its total mass-dimension $D_{\mathbb{S}}$ is determined by the number of external states $(\mathcal{E})$ and the number of spacetime dimensions, $D_{\mathbb{S}}=4-\mathcal{E}$, in the 4 d field theories. (ii).Consider the scattering amplitude $\mathbb{S}$ having scattering energy $E$ much larger than all the masses of the internal propagators as well as the masses of the external states. Thus, for the $E$-independent coupling constants contained in the amplitude $\mathbb{S}$, their total mass-dimension $D_{C}$ can be counted directly according to the type of vertices therein. Based on these, the total energy-power dependence $D_{E}$ of the amplitude $\mathbb{S}$ is given by $D_{E}=D_{\mathbb{S}}-D_{C}$. We note that for our following derivation in $3 \mathrm{~d}$ spacetime (or the general derivation in $d$-dimensional spacetime in appendix $\mathrm{B}$ ), we should modify the formula of $D_{\mathbb{S}}$ in point(i) accordingly. As for the point(ii), it should hold for any high energy scattering with energy $E$ much larger than the involved particle masses. The nontrivial energy-dependence from the polarization vectors (tensors) of the gauge bosons (gravitons) can be taken into account accordingly. With these in mind, we can construct the new power counting rules for the $3 \mathrm{~d}$ topologically massive gauge and gravity theories.

Consider a scattering $S$-matrix element $\mathbb{S}$ having $\mathcal{E}$ external states and $L$ loops $(L \geqslant 0)$ in the $(2+1)$ d spacetime. Thus, we can deduce that the amplitude $\mathbb{S}$ has a mass-dimension:

$$
D_{\mathbb{S}}=3-\frac{1}{2} \mathcal{E},
$$

where the number of external states $\mathcal{E}=\mathcal{E}_{B}+\mathcal{E}_{F}$, with $\mathcal{E}_{B}\left(\mathcal{E}_{F}\right)$ being the number of external bosonic (fermionic) states. We note that the above eq. (3.7) agrees to the $d=3$ case of our general formula (B.1) in appendix B. For the fermions, we only consider the SM fermions whose masses are much smaller than the scattering energy $E$. We denote the number of vertices of type- $j$ as $\mathcal{V}_{j}$. Each vertex of type- $j$ contains $d_{j}$ derivatives, $b_{j}$ bosonic lines,

\footnotetext{
${ }^{3}$ Weinberg's power counting rule was also extended previously $[12,52]$ to the $4 \mathrm{~d}$ gauge theories including the SM, the SM effective theory (SMEFT), and the electroweak chiral Lagrangian.
} 
and $f_{j}$ fermionic lines. Then, the energy-independent effective coupling constant in the amplitude $\mathbb{S}$ has its total mass-dimension given by

$$
D_{C}=\sum_{j} \mathcal{V}_{j}\left(3-d_{j}-\frac{1}{2} b_{j}-f_{j}\right) \text {. }
$$

For each Feynman diagram in the scattering amplitude $\mathbb{S}$, we denote the number of the internal lines as $I=I_{B}+I_{F}$ with $I_{B}\left(I_{F}\right)$ being the number of the internal bosonic (fermionic) lines. Thus, we have the following general relations:

$$
L=1+I-\mathcal{V}, \quad \sum_{j} \mathcal{V}_{j} b_{j}=2 I_{B}+\mathcal{E}_{B}, \quad \sum_{j} \mathcal{V}_{j} f_{j}=2 I_{F}+\mathcal{E}_{F},
$$

where $\mathcal{V}=\sum_{j} \mathcal{V}_{j}$ is the total number of vertices in a given Feynman diagram. With these, we can derive the following leading energy dependence $D_{E}=D_{\mathbb{S}}-D_{C}$ from eqs. (3.7)-(3.9):

$$
D_{E}=2(1-\mathcal{V})+L+\sum_{j} \mathcal{V}_{j}\left(d_{j}+\frac{1}{2} f_{j}\right) .
$$

Furthermore, we have the following relations:

$$
\sum_{j} \mathcal{V}_{j} d_{j}=\mathcal{V}_{d}, \quad \sum_{j} \mathcal{V}_{j} f_{j}=2 \mathcal{V}_{F}, \quad \mathcal{V}=\sum_{j} \mathcal{V}_{j}=\mathcal{V}_{3}+\mathcal{V}_{4}, \quad \mathcal{V}_{3}=\mathcal{V}_{d}+\mathcal{V}_{F}+\overline{\mathcal{V}}_{3}
$$

where $\mathcal{V}_{d}$ denotes the number of all cubic vertices including one partial derivative and $\overline{\mathcal{V}}_{3}$ denotes the number of bosonic cubic vertices having no partial derivative.

Then, we consider the topologically massive CS gauge theories. In such gauge theories, we have the relation $2 I+\mathcal{E}=3 \mathcal{V}_{3}+4 \mathcal{V}_{4}$. With these, we can derive the following power counting rule on the leading energy-power dependence:

$$
D_{E}=\left(\mathcal{E}_{A_{\mathrm{P}}}-\mathcal{E}_{v}\right)+\left(4-\mathcal{E}-\overline{\mathcal{V}}_{3}\right)-L
$$

where $\mathcal{E}_{A_{\mathrm{P}}}$ is the number of external gauge boson states with physical polarizations $\left(A_{\mathrm{P}}^{a}=\epsilon_{\mathrm{P}}^{\mu} A_{\mu}^{a}\right)$, and $\mathcal{E}_{v}$ denotes the number of external gauge bosons $v^{a}=v_{\mu} A^{a \mu}$. In eq. (3.12), the terms $\left(\mathcal{E}_{A_{\mathrm{P}}}-\mathcal{E}_{v}\right)$ arise from the high energy behaviors $\epsilon_{\mathrm{P}}^{\mu}=\mathcal{O}(E / m)$ and $v^{\mu}=\epsilon_{\mathrm{L}}^{\mu}-\epsilon_{\mathrm{S}}^{\mu}=\mathcal{O}(m / E)$.

For the sake of later applications, we consider the 3d topologically massive gravity (TMG) and derive the energy power counting rule for the graviton scattering amplitudes. The graviton self-interaction vertices from the gravitational CS term (5.1) (cf. section 5) always contain 3 partial derivatives and contribute to the leading energy dependence of the graviton scattering amplitudes, which correspond to $d_{j}=3$ and $f_{j}=0$ in eq. (3.10). Thus, we have $\sum_{j} \mathcal{V}_{j} d_{j}=3 \mathcal{V}_{d 3}$ and $\mathcal{V}=\mathcal{V}_{d 3}$ in such leading diagrams, where $\mathcal{V}_{d 3}$ denotes the number of vertices containing 3 partial derivatives. Hence, the leading energy dependence of the pure graviton scattering amplitudes in $(2+1) d$ arise from the Feynman diagrams containing the CS graviton vertices with 3 derivatives, and can be derived as follows:

$$
D_{E}=2 \mathcal{E}_{h_{\mathrm{P}}}+\left(2+\mathcal{V}_{d 3}+L\right),
$$


where $\mathcal{E}_{h_{\mathrm{P}}}$ denotes the number of external graviton states with physical polarizations $\left(h_{\mathrm{P}}=\right.$ $\left.\epsilon_{\mathrm{P}}^{\mu \nu} h_{\mu \nu}\right)$ and the physical graviton polarization tensor scales as $\epsilon_{\mathrm{P}}^{\mu \nu}=\mathcal{O}\left(E^{2} / m^{2}\right)$. For the leading tree-level diagrams composed of the cubic CS vertices with $d_{j}=3$, we derive a relation $\mathcal{E}_{h_{\mathrm{P}}}=2+\mathcal{V}_{d 3}$. Hence, using eq. (3.13), we can deduce the leading energy dependence of such tree-level diagrams:

$$
D_{E}^{0}=3 \mathcal{E}_{h_{P}}
$$

For instance, the leading 4-graviton scattering amplitudes of the TMG theory contain individual leading energy terms of $E^{12}$ at the tree level. We will analyze these further in section 5 .

\subsection{Energy cancellations for topological scattering amplitudes}

In this subsection, we will apply our power counting rule (3.12) to analyze the leading energy dependence of the pure gauge boson scattering amplitudes in the $3 \mathrm{~d}$ topological massive CS gauge theory. We also note that because the 3d CS theory is super-renormalizable, the leading energy dependence of a given amplitude is always given by the diagrams having $L=0$ (tree level) and $\overline{\mathcal{V}}_{3}=0$. Thus, given the external states of an amplitude, its maximal energy dependence is realized at tree level:

$$
D_{E}^{\max }=\left(\mathcal{E}_{A_{\mathrm{P}}}-\mathcal{E}_{v}\right)+(4-\mathcal{E})
$$

with $L=0$ and $\overline{\mathcal{V}}_{3}=0$.

According to eq. (3.15), the scattering amplitudes of pure gauge bosons $\left(A_{\mathrm{P}}^{a}\right)$ with the number of external states $\mathcal{E}=\mathcal{E}_{A_{\mathrm{P}}}=N$ and $\mathcal{E}_{v}=0$ can receive leading individual contributions of $\mathcal{O}\left(E^{4}\right)$ at the tree level. For the pure $A_{\mathrm{T}}^{a}$-amplitudes with $\mathcal{E}=\mathcal{E}_{A_{\mathrm{T}}}=N$ and $\mathcal{E}_{A_{\mathrm{P}}}=\mathcal{E}_{v}=0$, its individual leading contributions scale like $\mathcal{O}\left(E^{4-N}\right)$ at the tree level. With these, we find that our TET identity (3.5a) guarantees the energy cancellation in the $N$-gauge boson $\left(A_{\mathrm{P}}^{a}\right)$ scattering amplitude on its l.h.s.:

$$
E^{4} \rightarrow E^{4-N}
$$

This is because on the r.h.s. of eq. (3.5a) the corresponding pure $N$-gauge boson $\left(A_{\mathrm{T}}^{a}\right)$ amplitude scales as $\mathcal{O}\left(E^{4-N}\right)$ and the residual term $\mathcal{T}_{v}$ (with $\mathcal{E}_{v} \geqslant 1$ ) scales no more than $\mathcal{O}\left(E^{3-N}\right)$. We can readily generalize this result to up to $L$-loop level and deduce the following energy cancellations based on eq. (3.5a) and eq. (3.12):

$$
\Delta D_{E}=D_{E}\left[N A_{\mathrm{P}}^{a}\right]-D_{E}\left[N A_{\mathrm{T}}^{a}\right]=N .
$$

Hence, the TET identity (3.5) [or the TET (3.6)] provides a general mechanism which guarantees the nontrivial energy cancellations in eq. (3.16) or eq. (3.17).

Before concluding the current section 3, we discuss further the conversion of physical degrees of freedom during the $3 \mathrm{~d}$ topological mass-generation, in comparison with that realized during the $5 \mathrm{~d}$ geometric mass-generation under the Kaluza-Klein (KK) compactification. For the 3d gauge theories, before including the CS term, the massless gauge 
boson $A_{\mu}^{a}$ has only 1 physical transverse polarization state $A_{\mathrm{T}}^{a}$; while after including the CS term, the gauge boson acquires a topological mass and generates a single physical polarization state $A_{\mathrm{P}}^{a}$ (by absorbing the massless state $A_{\mathrm{T}}^{a}$ combined with the longitudinal state $A_{\mathrm{L}}^{a}$ ), without invoking the conventional spontaneous gauge symmetry breaking. Hence, this topological mass-generation mechanism leads to the conversion of the physical states: $A_{\mathrm{T}}^{a} \rightarrow A_{\mathrm{P}}^{a}$, which conserves the physical degree of freedom: $1=1$, as we explained earlier. In consequence, we observe that both the Lagrangians (2.1a)-(2.1b) and the gauge boson propagator (A.11a) indeed have a smooth massless limit $m \rightarrow 0$, which is similar to the massive KK gauge theories [13-16]. Based upon this mechanism of the topological massgeneration, we have newly established the TET (3.6) which connects a given $A_{\mathrm{P}}^{a}$-amplitude to the corresponding $A_{\mathrm{T}}^{a}$-amplitude under the high energy expansion.

In comparison, we note that the $5 \mathrm{~d}$ geometric mass-generation for the KK gauge bosons $A_{n}^{a \mu}$ is realized by absorbing ("eating") the corresponding 5 th components $A_{n}^{a 5}$ of the $5 \mathrm{~d}$ gauge fields $\widehat{A}_{M}^{a}$ at each KK level- $n$ [13-15]. The 5th components $A_{n}^{a 5}$ may be regarded as a kind of "geometric Goldstone bosons" due to the KK compactification, although they do not arise from a separate scalar Higgs potential and differs essentially from the conventional Higgs mechanism [6-10]. The 5d massless gauge boson $\widehat{A}_{M}^{a}$ has 3 physical transverse polarizations and after KK compactification each 4d massive KK gauge boson $A_{n}^{a \mu}$ has 2 transverse polarizations plus 1 longitudinal polarization (from absorbing $A_{n}^{a 5}$ ). So, the physical degrees of freedom are conserved before and after the KK mass generation: $3=2+1$; and this corresponds to the conversion of one physical degree of freedom at each KK level- $n: A_{5}^{a n} \rightarrow A_{\mathrm{L}}^{a n}$. This geometric mass generation of KK gauge bosons leads to the KK Equivalence Theorem for compactified gauge theories(KK GAET) which connects the high-energy scattering amplitudes of the longitudinal KK gauge bosons $A_{\mathrm{L}}^{a n}$ to that of the corresponding KK Goldstone bosons $A_{n}^{a 5}[13,16]^{4}$

\section{Topological scattering amplitudes and energy cancellations}

In this section, we present explicit calculations of the 4-particle scattering amplitudes in the topologically massive gauge theories including the Abelian QED (2.1a) and the non-Abelian TMYM theory $(2.1 \mathrm{~b})$. With these, we demonstrate explicitly the energy cancellation of $E^{2} \rightarrow E^{0}$ for the $A_{\mathrm{P}}$-amplitudes in the TMQED and the energy cancellation of $E^{4} \rightarrow E^{0}$ for the pure $A_{\mathrm{P}}^{a}$-amplitudes in the TMYM theory, under high energy expansion. Then, we verify the TET (3.6) for both the Abelian and non-Abelian CS gauge theories.

\subsection{Topologically massive QED and scattering amplitudes}

In this subsection, we consider two realizations of the topologically massive QED, namely, the topologically massive scalar QED (TMSQED) and the topologically massive spinor

\footnotetext{
${ }^{4}$ Besides, the study of the geometric mass-generation of $5 \mathrm{~d}$ KK gravitons and its gravitational equivalence theorem (GRET) were presented recently in ref. [27], where the KK graviton field $h_{n}^{\mu \nu}$ becomes massive by absorbing the scalar-component $h_{n}^{55}$ and vector-component $h_{n}^{\mu 5}$ from compactification of the 5d graviton field $\hat{h}^{M N}$. Note that before compactification the massless $5 \mathrm{~d}$ graviton $\hat{h}^{M N}$ has 5 physical degrees of freedom and after compactification the massive KK graviton $h_{n}^{\mu \nu}$ contains the physical states with helicities $\lambda= \pm 2, \pm 1,0$. We see that the physical degrees of freedom are conserved before and after the KK graviton mass-generation: $5=2+2+1$.
} 


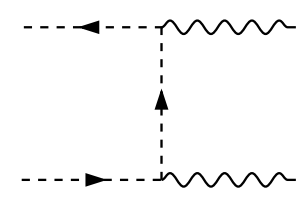

(a)

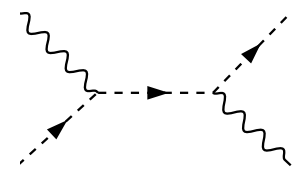

$(d)$

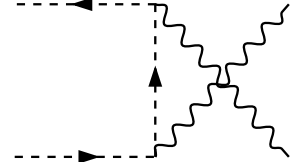

(b)

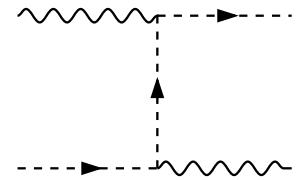

(e)

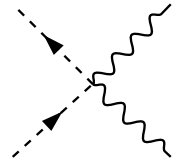

(c)

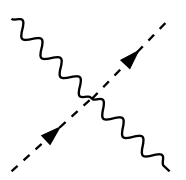

$(f)$

Figure 1. Feynman diagrams for the scattering processes $\phi^{-} \phi^{+} \rightarrow A_{\mathrm{P}} A_{\mathrm{P}}\left(\phi^{-} \phi^{+} \rightarrow A_{\mathrm{T}} A_{\mathrm{T}}\right)$ and $\phi^{-} A_{\mathrm{P}} \rightarrow \phi^{-} A_{\mathrm{P}}\left(\phi^{-} A_{\mathrm{T}} \rightarrow \phi^{-} A_{\mathrm{T}}\right)$ in $3 \mathrm{~d}$ topological massive scalar QED.

QED (TMQED). We will compute the scattering amplitudes in these two models and uncover the nontrivial energy cancellations in these amplitudes. Then, we will demonstrate explicitly that the TET (3.6) holds in each model.

\subsubsection{Scattering amplitudes of topologically massive scalar QED}

We first consider the TMSQED, which is composed by the scalar QED plus the ChernSimons term (2.1a). The Lagrangian contains a scalar sector:

$$
\mathcal{L}_{\mathrm{S}}=-\left(D_{\mu} \phi\right)^{*}\left(D^{\mu} \phi\right)-m_{\phi}^{2}|\phi|^{2}-\lambda|\phi|^{4}
$$

where we choose the metric tensor $\eta_{\mu \nu}=\eta^{\mu \nu}=\operatorname{diag}(-1,1,1)$ and denotes the complex scalar field by $\phi$. The covariant derivative is defined as $D_{\mu}=\partial_{\mu}+\mathrm{i} e A_{\mu}$. In the charge eigenstates, we have $\left(\phi^{-}, \phi^{+}\right)=\left(\phi, \phi^{*}\right)$, with $\phi^{-}\left(\phi^{+}\right)$denoting the scalar electron (scalar positron).

In the following, we compute and analyze two types of scattering processes $\phi^{-} \phi^{+} \rightarrow$ $A_{\mathrm{P}} A_{\mathrm{P}}\left(\phi^{-} \phi^{+} \rightarrow A_{\mathrm{T}} A_{\mathrm{T}}\right)$ and $\phi^{-} A_{\mathrm{P}} \rightarrow \phi^{-} A_{\mathrm{P}}\left(\phi^{-} A_{\mathrm{T}} \rightarrow \phi^{-} A_{\mathrm{T}}\right)$ at tree level, where the relevant Feynman diagrams are shown in figure 1.

For the annihilation processes $\phi^{-} \phi^{+} \rightarrow A_{\mathrm{P}} A_{\mathrm{P}}$ and $\phi^{-} \phi^{+} \rightarrow A_{\mathrm{T}} A_{\mathrm{T}}$, we find that under the high energy expansion and by using the power counting rule (3.12), the scattering amplitude $\mathcal{T}\left[\phi^{-} \phi^{+} \rightarrow A_{\mathrm{P}} A_{\mathrm{P}}\right]$ scales as $E^{2}$, while the scattering amplitude $\mathcal{T}\left[\phi^{-} \phi^{+} \rightarrow\right.$ $\left.A_{\mathrm{T}} A_{\mathrm{T}}\right]$ scales as $E^{0}$. Thus, we can make high energy expansions for both amplitudes as follows:

$$
\begin{aligned}
& \mathcal{T}\left[\phi^{-} \phi^{+} \rightarrow A_{\mathrm{P}} A_{\mathrm{P}}\right]=\mathcal{T}_{\mathrm{PP}}^{(2)} \bar{E}^{2}+\mathcal{T}_{\mathrm{PP}}^{(1)} \bar{E}^{1}+\mathcal{T}_{\mathrm{PP}}^{(0)} \bar{E}^{0}+\mathcal{O}\left(\bar{E}^{-1}\right), \\
& \mathcal{T}\left[\phi^{-} \phi^{+} \rightarrow A_{\mathrm{T}} A_{\mathrm{T}}\right]=\mathcal{T}_{\mathrm{TT}}^{(0)} \bar{E}^{0}+\mathcal{O}\left(\bar{E}^{-1}\right),
\end{aligned}
$$

where $\bar{E}=E / m$ and $E$ denotes the energy of the scalar electron (positron). For simplicity, we set the scalar mass $m_{\phi} \simeq 0$. The amplitude $\mathcal{T}\left[\phi^{-} \phi^{+} \rightarrow A_{\mathrm{P}} A_{\mathrm{P}}\right]$ contains the contributions of the Feynman diagrams $(a)-(c)$ of figure 1. According to eq. (4.2a), we compute the amplitude at each order of the high energy expansion, which is given by the sum of 


\begin{tabular}{|c|c|c|c|c|c|}
\hline Amplitude & $\times \bar{E}^{2}$ & $\times \bar{E}^{1}$ & Amplitude & $\times \bar{E}^{2}$ & $\times \bar{E}^{1}$ \\
\hline $\mathcal{T}_{\mathrm{PP}}[(a)]$ & $-e^{2}\left(1+c_{\theta}\right)$ & $-\mathrm{i} 2 e^{2} s_{\theta}$ & $\mathcal{T}_{\phi \mathrm{P}}[(d)]$ & $-2 e^{2}$ & 0 \\
\hline $\mathcal{T}_{\mathrm{PP}}[(b)]$ & $-e^{2}\left(1-c_{\theta}\right)$ & $\mathrm{i} 2 e^{2} s_{\theta}$ & $\mathcal{T}_{\phi \mathrm{P}}[(e)]$ & $e^{2}\left(1+c_{\theta}\right)$ & $\mathrm{i} 2 e^{2} s_{\theta}$ \\
\hline $\mathcal{T}_{\mathrm{PP}}[(c)]$ & $2 e^{2}$ & 0 & $\mathcal{T}_{\phi \mathrm{P}}[(f)]$ & $e^{2}\left(1-c_{\theta}\right)$ & $-\mathrm{i} 2 e^{2} s_{\theta}$ \\
\hline Sum & 0 & 0 & Sum & 0 & 0 \\
\hline
\end{tabular}

Table 1. Energy cancellations in the scattering amplitudes of 3d topologically massive scalar QED, $\mathcal{T}\left[\phi^{-} \phi^{+} \rightarrow A_{\mathrm{P}}^{a} A_{\mathrm{P}}^{a}\right]=\mathcal{T}_{\mathrm{PP}}[(a)]+\mathcal{T}_{\mathrm{PP}}[(b)]+\mathcal{T}_{\mathrm{PP}}[(c)]$ and $\mathcal{T}\left[\phi^{-} A_{\mathrm{P}}^{a} \rightarrow \phi^{-} A_{\mathrm{P}}^{a}\right]=\mathcal{T}_{\phi \mathrm{P}}[(d)]+\mathcal{T}_{\phi \mathrm{P}}[(e)]+$ $\mathcal{T}_{\phi \mathrm{P}}[(f)]$, where $\bar{E}=E / m$ and $\left(s_{\theta}, c_{\theta}\right)=(\sin \theta, \cos \theta)$ with $\theta$ being the scattering angle. Each full amplitude equals the sum of individual diagrams $(a)+(b)+(c)$ and $(c)+(d)+(e)$, respectively, as shown in figure 1.

the three diagrams $(a)-(c)$. As shown in table 1 , we demonstrate explicitly that the sum of diagrams $(a)-(c)$ vanishes at the $\mathcal{O}\left(\bar{E}^{2}\right)$ and $\mathcal{O}\left(\bar{E}^{1}\right)$ :

$$
\begin{aligned}
& \mathcal{T}_{\mathrm{PP}}^{(2)}[(a)+(b)+(c)]=0, \\
& \mathcal{T}_{\mathrm{PP}}^{(1)}[(a)+(b)+(c)]=0 .
\end{aligned}
$$

Furthermore, we compute both amplitudes $\mathcal{T}\left[\phi^{-} \phi^{+} \rightarrow A_{\mathrm{P}} A_{\mathrm{P}}\right]$ and $\mathcal{T}\left[\phi^{-} \phi^{+} \rightarrow A_{\mathrm{T}} A_{\mathrm{T}}\right]$ at the $\mathcal{O}\left(\bar{E}^{0}\right)$ and obtain:

$$
\mathcal{T}_{\mathrm{PP}}^{(0)}\left[\phi^{-} \phi^{+} \rightarrow A_{\mathrm{P}} A_{\mathrm{P}}\right]=\frac{1}{2} \mathcal{T}_{\mathrm{TT}}^{(0)}\left[\phi^{-} \phi^{+} \rightarrow A_{\mathrm{T}} A_{\mathrm{T}}\right]=e^{2} .
$$

Without losing generality, we set the spin $\mathfrak{s}=\widetilde{m} / m=+1$ in the above calculations and afterwards.

Similarly, we compute the Compton scattering amplitudes $\mathcal{T}\left[\phi^{-} A_{\mathrm{P}} \rightarrow \phi^{-} A_{\mathrm{P}}\right]$ and $\mathcal{T}\left[\phi^{-} A_{\mathrm{T}} \rightarrow \phi^{-} A_{\mathrm{T}}\right]$. Then, we make the following high energy expansions for both amplitudes:

$$
\begin{aligned}
& \mathcal{T}\left[\phi^{-} A_{\mathrm{P}} \rightarrow \phi^{-} A_{\mathrm{P}}\right]=\mathcal{T}_{\phi \mathrm{P}}^{(2)} \bar{E}^{2}+\mathcal{T}_{\phi \mathrm{P}}^{(1)} \bar{E}^{1}+\mathcal{T}_{\phi \mathrm{P}}^{(0)} \bar{E}^{0}+\mathcal{O}\left(\bar{E}^{-1}\right), \\
& \mathcal{T}\left[\phi^{-} A_{\mathrm{T}} \rightarrow \phi^{-} A_{\mathrm{T}}\right]=\mathcal{T}_{\phi \mathrm{T}}^{(0)} \bar{E}^{0}+\mathcal{O}\left(\bar{E}^{-1}\right) .
\end{aligned}
$$

As shown in table 1 , we demonstrate explicitly that the sum of the three diagrams $(d)-(f)$ of figure 1 vanishes at the $\mathcal{O}\left(\bar{E}^{2}\right)$ and $\mathcal{O}\left(\bar{E}^{1}\right)$ :

$$
\begin{aligned}
& \mathcal{T}_{\phi \mathrm{P}}^{(2)}[(d)+(e)+(f)]=0, \\
& \mathcal{T}_{\phi \mathrm{P}}^{(1)}[(d)+(e)+(f)]=0 .
\end{aligned}
$$

Moreover, we find that both amplitudes $\mathcal{T}\left[\phi^{-} A_{\mathrm{P}} \rightarrow \phi^{-} A_{\mathrm{P}}\right]$ and $\mathcal{T}\left[\phi^{-} A_{\mathrm{T}} \rightarrow \phi^{-} A_{\mathrm{T}}\right]$ are nonzero and equal at the $\mathcal{O}\left(\bar{E}^{0}\right)$ :

$$
\mathcal{T}_{\phi \mathrm{P}}^{(0)}\left[\phi^{-} A_{\mathrm{P}} \rightarrow \phi^{-} A_{\mathrm{P}}\right]=\frac{1}{2} \mathcal{T}_{\phi \mathrm{T}}^{(0)}\left[\phi^{-} A_{\mathrm{T}} \rightarrow \phi^{-} A_{\mathrm{T}}\right]=-e^{2}
$$

Finally, from eqs. (4.3) and (4.6) together with eqs. (4.4) and (4.7), we derive

$$
\begin{aligned}
& \mathcal{T}\left[\phi^{-} \phi^{+} \rightarrow A_{\mathrm{P}} A_{\mathrm{P}}\right]=\frac{1}{2} \mathcal{T}\left[\phi^{-} \phi^{+} \rightarrow A_{\mathrm{T}} A_{\mathrm{T}}\right]+\mathcal{O}\left(\frac{m}{E}\right), \\
& \mathcal{T}\left[\phi^{-} A_{\mathrm{P}} \rightarrow \phi^{-} A_{\mathrm{P}}\right]=\frac{1}{2} \mathcal{T}\left[\phi^{-} A_{\mathrm{T}} \rightarrow \phi^{-} A_{\mathrm{T}}\right]+\mathcal{O}\left(\frac{m}{E}\right),
\end{aligned}
$$

which explicitly verify the TET (3.6) for the topologically massive scalar QED. 


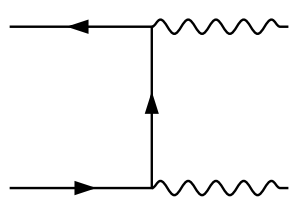

(a)

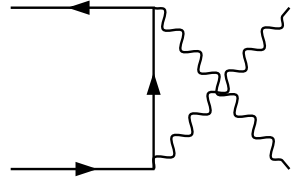

(b)

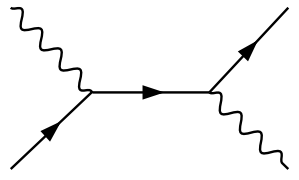

(c)

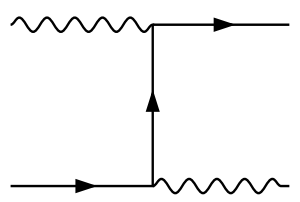

(d)

Figure 2. Scattering processes $e^{+} e^{-} \rightarrow A_{\mathrm{P}} A_{\mathrm{P}}\left(e^{+} e^{-} \rightarrow A_{\mathrm{T}} A_{\mathrm{T}}\right)$ via Feynman diagrams $(a)-(b)$ and $e^{-} A_{\mathrm{P}} \rightarrow e^{-} A_{\mathrm{P}}\left(e^{-} A_{\mathrm{T}} \rightarrow e^{-} A_{\mathrm{T}}\right)$ via Feynman diagrams $(c)-(d)$ in $3 \mathrm{~d}$ topologically massive spinor QED.

\subsubsection{Scattering amplitudes of topologically massive spinor QED}

In this subsection, we consider the topologically massive QED (TMQED) which includes the gauge sector Lagrangian (2.1a) (with Chern-Simons term) and the following matter Lagrangian,

$$
\mathcal{L}_{f}=\bar{\psi}\left(\gamma^{\mu} D_{\mu}-m_{f}\right) \psi
$$

where the covariant derivative is defined as $D_{\mu}=\partial_{\mu}+\mathrm{i} e A_{\mu}$ and the gamma matrices are given by $\left(\gamma^{0}, \gamma^{1}, \gamma^{2}\right)=\left(\mathrm{i} \sigma^{2}, \sigma^{1}, \sigma^{3}\right)$. We define the $3 \mathrm{~d}$ Dirac spinors and solve the $3 \mathrm{~d}$ Dirac equation in appendix $\mathrm{C}$.

Then, we analyze the amplitudes of the annihilation process $e^{+} e^{-} \rightarrow A_{\mathrm{P}} A_{\mathrm{P}}\left(e^{+} e^{-} \rightarrow\right.$ $\left.A_{\mathrm{T}} A_{\mathrm{T}}\right)$ and the Compton scattering $e^{-} A_{\mathrm{P}} \rightarrow e^{-} A_{\mathrm{P}}\left(e^{-} A_{\mathrm{T}} \rightarrow e^{-} A_{\mathrm{T}}\right)$. The relevant Feynman diagrams at tree level are shown in figure 2. Using the power counting rule (3.12), we find that the scattering amplitudes $\mathcal{T}\left[e^{+} e^{-} \rightarrow A_{\mathrm{P}} A_{\mathrm{P}}\right]$ and $\mathcal{T}\left[e^{-} A_{\mathrm{P}} \rightarrow e^{-} A_{\mathrm{P}}\right]$ have leading contributions scale as $E^{2}$, while the scattering amplitudes $\mathcal{T}\left[e^{+} e^{-} \rightarrow A_{\mathrm{T}} A_{\mathrm{T}}\right]$ and $\mathcal{T}\left[e^{-} A_{\mathrm{T}} \rightarrow e^{-} A_{\mathrm{T}}\right]$ have leading contributions scale as $E^{0}$. Thus, we can make the following high energy expansions:

$$
\begin{aligned}
& \mathcal{T}\left[e^{-} e^{+} \rightarrow A_{\mathrm{P}} A_{\mathrm{P}}\right]=\mathcal{T}_{\mathrm{PP}}^{(2)} \bar{E}^{2}+\mathcal{T}_{\mathrm{PP}}^{(1)} \bar{E}^{1}+\mathcal{T}_{\mathrm{PP}}^{(0)} \bar{E}^{0}+\mathcal{O}\left(\bar{E}^{-1}\right), \\
& \mathcal{T}\left[e^{-} e^{+} \rightarrow A_{\mathrm{T}} A_{\mathrm{T}}\right]=\mathcal{T}_{\mathrm{TT}}^{(0)} \bar{E}^{0}+\mathcal{O}\left(\bar{E}^{-1}\right), \\
& \mathcal{T}\left[e^{-} A_{\mathrm{P}} \rightarrow e^{-} A_{\mathrm{P}}\right]=\mathcal{T}_{e \mathrm{P}}^{(2)} \bar{E}^{2}+\mathcal{T}_{e \mathrm{P}}^{(1)} \bar{E}^{1}+\mathcal{T}_{e \mathrm{P}}^{(0)} \bar{E}^{0}+\mathcal{O}\left(\bar{E}^{-1}\right), \\
& \mathcal{T}\left[e^{-} A_{\mathrm{T}} \rightarrow e^{-} A_{\mathrm{T}}\right]=\mathcal{T}_{e \mathrm{~T}}^{(0)} \bar{E}^{0}+\mathcal{O}\left(\bar{E}^{-1}\right),
\end{aligned}
$$

where $\bar{E}=E / m$ and $E$ denotes the energy of the incoming electron (positron). For simplicity, we set the electron mass $m_{e} \simeq 0$.

Then, we explicitly compute the above scattering amplitudes. We find that all the $\mathcal{O}\left(E^{2}\right)$ and $\mathcal{O}\left(E^{1}\right)$ terms cancel exactly in each amplitude and the final results actually behave as $\mathcal{O}\left(E^{0}\right)$. We present these cancellations explicitly in table 2. Hence, we have

$$
\begin{array}{ll}
\mathcal{T}_{\mathrm{PP}}^{(2)}[(a)+(b)]=0, & \mathcal{T}_{\mathrm{PP}}^{(1)}[(a)+(b)]=0 ; \\
\mathcal{T}_{e \mathrm{P}}^{(2)}[(c)+(d)]=0, & \mathcal{T}_{e \mathrm{P}}^{(1)}[(c)+(d)]=0 .
\end{array}
$$




\begin{tabular}{|c|c|c|c|c|c|}
\hline Amplitude & $\times \bar{E}^{2}$ & $\times \bar{E}^{1}$ & Amplitude & $\times \bar{E}^{2}$ & $\times \bar{E}^{1}$ \\
\hline $\mathcal{T}_{\mathrm{PP}}[(a)]$ & $\mathrm{i} e^{2} s_{\theta}$ & $2 e^{2} c_{\theta}$ & $\mathcal{T}_{e \mathrm{P}}[(c)]$ & $\frac{\mathrm{i} 2 e^{2}\left(1+c_{\theta}\right)\left(1+s_{\theta}\right)^{1 / 2}}{1+c_{\theta}+s_{\theta}}$ & $-\frac{2 e^{2} s_{\theta}\left(1+s_{\theta}\right)^{1 / 2}}{1+c_{\theta}+s_{\theta}}$ \\
\hline $\mathcal{T}_{\mathrm{PP}}[(b)]$ & $-\mathrm{i} e^{2} s_{\theta}$ & $-2 e^{2} c_{\theta}$ & $\mathcal{T}_{e \mathrm{P}}[(d)]$ & $-\frac{\mathrm{i} 2 e^{2}\left(1+c_{\theta}\right)\left(1+s_{\theta}\right)^{1 / 2}}{1+c_{\theta}+s_{\theta}}$ & $\frac{2 e^{2} s_{\theta}\left(1+s_{\theta}\right)^{1 / 2}}{1+c_{\theta}+s_{\theta}}$ \\
\hline Sum & 0 & 0 & Sum & 0 & 0 \\
\hline
\end{tabular}

Table 2. Energy cancellations in the scattering amplitudes of 3d topologically massive spinor QED, $\mathcal{T}\left[e^{+} e^{-} \rightarrow A_{\mathrm{P}} A_{\mathrm{P}}\right]=\mathcal{T}_{\mathrm{PP}}[(a)]+\mathcal{T}_{\mathrm{PP}}[(b)]$ and $\mathcal{T}\left[e^{-} A_{\mathrm{P}} \rightarrow e^{-} A_{\mathrm{P}}\right]=\mathcal{T}_{e \mathrm{P}}[(c)]+\mathcal{T}_{e \mathrm{P}}[(d)]$, where the notations are defined as $\bar{E}=E / m$ and $\left(s_{\theta}, c_{\theta}\right)=(\sin \theta, \cos \theta)$ with $\theta$ denoting the scattering angle. Each full amplitude equals the sum of individual diagrams $(a)+(b)$ and $(c)+(d)$, respectively, as shown in figure 2 .

Finally, we derive the remaining amplitudes of $\mathcal{O}\left(E^{0}\right)$ as follows:

$$
\begin{aligned}
& \mathcal{T}_{\mathrm{PP}}^{(0)}\left[e^{-} e^{+} \rightarrow A_{\mathrm{P}} A_{\mathrm{P}}\right]=\frac{1}{2} \mathcal{T}_{\mathrm{TT}}^{(0)}\left[e^{-} e^{+} \rightarrow A_{\mathrm{T}} A_{\mathrm{T}}\right]=\mathrm{i} e^{2} \cot \theta, \\
& \mathcal{T}_{e \mathrm{P}}^{(0)}\left[e^{-} A_{\mathrm{P}} \rightarrow e^{-} A_{\mathrm{P}}\right]=\frac{1}{2} \mathcal{T}_{e \mathrm{~T}}^{(0)}\left[e^{-} A_{\mathrm{T}} \rightarrow e^{-} A_{\mathrm{T}}\right]=\mathrm{i} e^{2} \frac{\left(3+c_{\theta}\right)\left(1+c_{\theta}+s_{\theta}\right)}{4\left(1+c_{\theta}\right)\left(1+s_{\theta}\right)^{\frac{1}{2}}} .
\end{aligned}
$$

For completeness, we also summarize in appendix D the full tree-level amplitudes (without high energy expansion) for the scattering processes discussed above and in sections 4.1 and 4.2.1. These exact formulas can provide self-consistency checks for the corresponding expanded scattering amplitudes given in the main text and will also be useful for future studies.

From the above eqs. (4.12a)-(4.12b), we deduce the following relations under the high energy expansion:

$$
\begin{aligned}
\mathcal{T}\left[e^{-} e^{+} \rightarrow A_{\mathrm{P}} A_{\mathrm{P}}\right] & =\frac{1}{2} \mathcal{T}\left[e^{-} e^{+} \rightarrow A_{\mathrm{T}} A_{\mathrm{T}}\right]+\mathcal{O}\left(\frac{m}{E}\right), \\
\mathcal{T}\left[e^{-} A_{\mathrm{P}} \rightarrow e^{-} A_{\mathrm{P}}\right] & =\frac{1}{2} \mathcal{T}\left[e^{-} A_{\mathrm{T}} \rightarrow e^{-} A_{\mathrm{T}}\right]+\mathcal{O}\left(\frac{m}{E}\right) .
\end{aligned}
$$

These verify explicitly that the TET (3.6) does hold, as expected from our general formulation of the TET in section 3.1. We observe that the TET identity (3.5) [or the TET (3.6)] provides a general mechanism which guarantees the exact energy cancellations of the $\mathcal{O}\left(E^{2}\right)$ and $\mathcal{O}\left(E^{1}\right)$ contributions in the $A_{\mathrm{P}}^{a}$-amplitude and matches the corresponding $A_{\mathrm{T}}^{a}$-amplitude of $\mathcal{O}\left(E^{0}\right)$.

Before concluding this subsection, we further present an exact verification of the TET identity (3.4) or (3.5a) without taking the high energy limit and by considering the simplest case of $N=1$. For the scattering process $e^{-} e^{+} \rightarrow A_{\mathrm{P}} A_{\mathrm{P}}$, we apply the TET identity (3.5) to just one external state of $A_{\mathrm{P}}$ :

$$
\mathcal{T}\left[e^{-} e^{+} \rightarrow A_{\mathrm{P}} A_{\mathrm{P}}\right]=\mathcal{T}\left[e^{-} e^{+} \rightarrow \tilde{A}_{\mathrm{T}} A_{\mathrm{P}}\right]+\mathcal{T}\left[e^{-} e^{+} \rightarrow \tilde{v} A_{\mathrm{P}}\right],
$$

where $\tilde{A}_{\mathrm{T}}=\frac{1}{\sqrt{2}} A_{\mathrm{T}}$ and $\tilde{v}=\frac{1}{\sqrt{2}} v=\frac{1}{\sqrt{2}} v^{\mu} A_{\mu}$. Using the basic relation of polarization vectors in eq. (3.2), we can rewrite the above TET identy (4.14) as follows:

$$
\mathcal{T}\left[e^{-} e^{+} \rightarrow A_{\mathrm{S}} A_{\mathrm{P}}\right]=0
$$


where $A_{\mathrm{S}}=\epsilon_{\mathrm{S}}^{\mu} A_{\mu}$ is the unphysical scalar polarization state of the photon. As we explained above eq. (3.2), the gauge-fixing function in momentum space can be expressed as $\mathcal{F}=$ $-\mathrm{i} m A_{\mathrm{S}}$. Thus, the above TET identity (4.15) is equivalent to

$$
\mathcal{T}\left[e^{-} e^{+} \rightarrow \mathcal{F} A_{\mathrm{P}}\right]=0
$$

which is just the simplest $N=1$ case of the Slavnov-Taylor-type identity (3.1). Hence, to verify the TET identity (3.5) in the case of $N=1$, we only need to prove explicitly that the identity (4.15) holds at the tree level.

The tree-level scattering process $e^{-} e^{+} \rightarrow A_{\mathrm{S}} A_{\mathrm{P}}$ contains the same type of diagrams $(a)$ - $(b)$ via $(t, u)$-channels, as shown in figure 2 . Then, we compute directly the contributions of the $(t, u)$-channels as follows:

$$
\mathcal{T}_{t}\left[e^{-} e^{+} \rightarrow A_{\mathrm{S}} A_{\mathrm{P}}\right]=-\mathcal{T}_{u}\left[e^{-} e^{+} \rightarrow A_{\mathrm{S}} A_{\mathrm{P}}\right]=\sqrt{2} e^{2}\left(\mathrm{i} \bar{E}^{2} s_{\theta}+\bar{E} c_{\theta}\right),
$$

which ensures that the full amplitude vanishes:

$$
\mathcal{T}\left[e^{-} e^{+} \rightarrow A_{\mathrm{S}} A_{\mathrm{P}}\right]=\mathcal{T}_{t}+\mathcal{T}_{u}=0 .
$$

This explicitly verifies the TET identity (4.15) [and thus the TET identity (4.14)] for the case of $N=1$, without taking the high energy limit.

\subsection{Topologically massive QCD and scattering amplitudes}

In this subsection, we study scattering amplitudes in the $3 \mathrm{~d}$ topologically massive QCD (TMQCD) with non-Abelian gauge group $\mathrm{SU}(N)$. This is also called the topologically massive YM (TMYM) theory in section 2 for the pure gauge sector without matter fields. We will not discriminate these two terminologies hereafter. The Lagrangian of the TMQCD can be written as follows:

$$
\mathcal{L}=-\frac{1}{4}\left(F_{\mu \nu}^{a}\right)^{2}+\frac{m}{2} \varepsilon^{\mu \nu \rho} A_{\mu}^{a} \partial_{\nu} A_{\rho}^{a}+\frac{g m}{6} C^{a b c} \varepsilon^{\mu \nu \rho} A_{\mu}^{a} A_{\nu}^{b} A_{\rho}^{c}+\sum_{i, j=1}^{N} \bar{\psi}_{i}\left(\gamma_{\mu} D_{i j}^{\mu}-m_{q} \delta_{i j}\right) \psi_{j}
$$

where $D_{i j}^{\mu}=\delta_{i j} \partial^{\mu}-\mathrm{i} g A^{a \mu} T_{i j}^{a}$ and $(i, j)$ denote the color indices of the quarks. We will compute the scattering amplitudes of the quark-antiquark annihilation and the pure gauge boson scattering, from which uncover the nontrivial energy cancellations. Then, we will demonstrate explicitly that the TET (3.6) holds for the non-Abelian TMQCD.

\subsubsection{Scattering amplitudes of quark-antiquark annihilation}

In this subsection, we analyze the scattering amplitudes of quark-antiquark annihilation processes $q \bar{q} \rightarrow A_{\mathrm{P}}^{a} A_{\mathrm{P}}^{b}$ and $q \bar{q} \rightarrow A_{\mathrm{T}}^{a} A_{\mathrm{T}}^{b}$, which include three Feynman diagrams as shown in figure 3. The non-Abelian cubic gluon vertex generates the $s$-channel diagram of figure $3(a)$ which is absent in the $e^{-} e^{+}$annihilation process of the TMQED as shown figure 2(a)-(b).

Applying the power counting rule (3.12), we find that the high-energy scattering amplitude $\mathcal{T}\left[q_{i} \bar{q}_{j} \rightarrow A_{\mathrm{P}}^{a} A_{\mathrm{P}}^{b}\right]$ has leading contributions scale as $E^{2}$, while the amplitude 


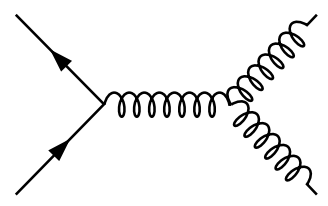

(a)

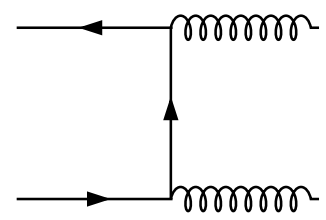

(b)

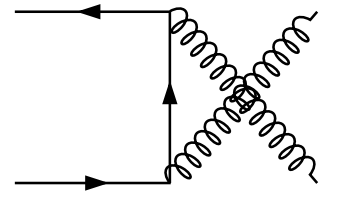

(c)

Figure 3. Scattering processes of quark-antiquark annihilation into two gluons, $q_{i} \bar{q}_{j} \rightarrow A_{\mathrm{P}}^{a} A_{\mathrm{P}}^{b}$ $\left(q_{i} \bar{q}_{j} \rightarrow A_{\mathrm{T}}^{a} A_{\mathrm{T}}^{b}\right)$ in the $3 \mathrm{~d}$ topologically massive QCD.

$\mathcal{T}\left[q_{i} \bar{q}_{j} \rightarrow A_{\mathrm{T}}^{a} A_{\mathrm{T}}^{b}\right]$ scales as $E^{0}$. Thus, we can make the following high energy expansions:

$$
\begin{aligned}
& \mathcal{T}\left[q_{i} \bar{q}_{j} \rightarrow A_{\mathrm{P}}^{a} A_{\mathrm{P}}^{b}\right]=\mathcal{T}_{\mathrm{PP}}^{(2)} \bar{E}^{2}+\mathcal{T}_{\mathrm{PP}}^{(1)} \bar{E}^{1}+\mathcal{T}_{\mathrm{PP}}^{(0)} \bar{E}^{0}+\mathcal{O}\left(\bar{E}^{-1}\right), \\
& \mathcal{T}\left[q_{i} \bar{q}_{j} \rightarrow A_{\mathrm{T}}^{a} A_{\mathrm{T}}^{b}\right]=\mathcal{T}_{\mathrm{TT}}^{(0)} \bar{E}^{0}+\mathcal{O}\left(\bar{E}^{-1}\right),
\end{aligned}
$$

where $\bar{E}=E / m$ and $E$ denotes the energy of the incoming quark (anti-quark). For simplicity, we set the quark mass $m_{q} \simeq 0$. Then, we explicitly compute these scattering amplitudes, and find that summed contributions in each amplitude cancel exactly at $\mathcal{O}\left(E^{2}\right)$ and $\mathcal{O}\left(E^{1}\right)$, respectively. The final net results could only behave as $\mathcal{O}\left(E^{0}\right)$. We present these cancellations explicitly in table 3 . From these, we deduce

$$
\begin{aligned}
& \mathcal{T}_{\mathrm{PP}}^{(2)}[(a)+(b)+(c)]=0, \\
& \mathcal{T}_{\mathrm{PP}}^{(1)}[(a)+(b)+(c)]=0,
\end{aligned}
$$

where we have applied the commutation relation $\left[T^{a}, T^{b}\right]=\mathrm{i} C^{a b c} T^{c}$ to the sum of the diagrams $(b)+(c)$, which further cancels the contribution of the diagram $(a)$ at $\mathcal{O}\left(E^{2}\right)$ and $\mathcal{O}\left(E^{1}\right)$ respectively.

Next, we compute the remaining $q \bar{q}$ annihilation amplitudes at $\mathcal{O}\left(E^{0}\right)$ and derive the following results:

$$
\mathcal{T}_{\mathrm{PP}}^{(0)}\left[q_{i} \bar{q}_{j} \rightarrow A_{\mathrm{P}}^{a} A_{\mathrm{P}}^{b}\right]=\frac{1}{2} \mathcal{T}_{\mathrm{TT}}^{(0)}\left[q_{i} \bar{q}_{j} \rightarrow A_{\mathrm{T}}^{a} A_{\mathrm{T}}^{b}\right]=\frac{\mathrm{i} g^{2}}{4}\left[\frac{s_{2 \theta}}{1+c_{\theta}}\left(T_{j k}^{a} T_{k i}^{b}\right)+\frac{s_{2 \theta}}{1-c_{\theta}}\left(T_{j k}^{b} T_{k i}^{a}\right)\right] .
$$

We may further define the color-singlet states of the $\mathrm{SU}(N)$ gauge group:

$$
\begin{aligned}
|0\rangle_{q} & =\frac{1}{\sqrt{N}} \sum_{j=1}^{N}\left|q_{j} \bar{q}_{j}\right\rangle, \\
|0\rangle_{A_{\mathrm{P}}} & =\frac{1}{\sqrt{2\left(N^{2}-1\right)}} \sum_{a=1}^{N^{2}-1}\left|A_{\mathrm{P}}^{a} A_{\mathrm{P}}^{a}\right\rangle, \\
|0\rangle_{A_{\mathrm{T}}} & =\frac{1}{\sqrt{2\left(N^{2}-1\right)}} \sum_{a=1}^{N^{2}-1}\left|A_{\mathrm{T}}^{a} A_{\mathrm{T}}^{a}\right\rangle .
\end{aligned}
$$

Then, we compute the $q \bar{q}$ annihilation amplitudes of the color-singlet states at $\mathcal{O}\left(E^{0}\right)$ :

$$
\mathcal{T}_{\mathrm{PP}}^{(0)}\left[|0\rangle_{q} \rightarrow|0\rangle_{A_{\mathrm{P}}}\right]=\frac{1}{2} \mathcal{T}_{\mathrm{TT}}^{(0)}\left[|0\rangle_{q} \rightarrow|0\rangle_{A_{\mathrm{T}}}\right]=\mathrm{i} g^{2} f(N) \cot \theta
$$




\begin{tabular}{|c|c|c|c|c|}
\hline Amplitude & $\mathcal{T}_{\mathrm{PP}}[(a)]$ & $\mathcal{T}_{\mathrm{PP}}[(b)]$ & $\mathcal{T}_{\mathrm{PP}}[(c)]$ & Sum \\
\hline$\times \bar{E}^{2}$ & $g^{2} s_{\theta} C^{a b c} T^{c}$ & $\mathrm{i} g^{2} s_{\theta} T^{a} T^{b}$ & $-\mathrm{i} g^{2} s_{\theta} T^{b} T^{a}$ & 0 \\
\hline$\times \bar{E}^{1}$ & $-\mathrm{i} 2 g^{2} c_{\theta} C^{a b c} T^{c}$ & $2 g^{2} c_{\theta} T^{a} T^{b}$ & $-2 g^{2} c_{\theta} T^{b} T^{a}$ & 0 \\
\hline
\end{tabular}

Table 3. Energy cancellations in the scattering amplitude $\mathcal{T}\left[q_{i} \bar{q}_{j} \rightarrow A_{\mathrm{P}}^{a} A_{\mathrm{P}}^{b}\right]=\mathcal{T}_{\mathrm{PP}}[(a)]+\mathcal{T}_{\mathrm{PP}}[(b)]+$ $\mathcal{T}_{\mathrm{PP}}[(c)]$ of $3 \mathrm{~d}$ topologically massive QCD, where the relevant Feynman diagrams $(a)-(c)$ are shown in figure 3 .

where we have defined the function $f(N)=\frac{1}{2 \sqrt{2}} \sqrt{\left(N^{2}-1\right) / N}$. We note that for the colorsinglet initial and final states, the $s$-channel contribution vanishes due to $C^{a a c}=0$, and the sum of the $(t, u)$-channel contributions just differs from the Abelian case of TMQED by an overall factor $\left(g^{2} / e^{2}\right) f(N)$. This relation holds even without making the high energy expansion, namely,

$$
\begin{aligned}
& \mathcal{T}_{\mathrm{PP}}\left[|0\rangle_{q} \rightarrow|0\rangle_{A_{\mathrm{P}}}\right]=\frac{g^{2}}{e^{2}} f(N) \mathcal{T}\left[e^{-} e^{+} \rightarrow A_{\mathrm{P}} A_{\mathrm{P}}\right], \\
& \mathcal{T}_{\mathrm{TT}}\left[|0\rangle_{q} \rightarrow|0\rangle_{A_{\mathrm{T}}}\right]=\frac{g^{2}}{e^{2}} f(N) \mathcal{T}\left[e^{-} e^{+} \rightarrow A_{\mathrm{T}} A_{\mathrm{T}}\right] .
\end{aligned}
$$

After the high energy expansion, only the $\mathcal{O}\left(E^{0}\right)$ amplitudes survive for the TMQCD and TMQED, as shown in eq. (4.24) and eq. (4.12a) which obey the above relations (4.25).

Finally, from eqs. (4.20)(4.21) and eqs. (4.22)(4.24), we derive the following equivalence relation under the high energy expansion:

$$
\mathcal{T}\left[q_{i} \bar{q}_{j} \rightarrow A_{\mathrm{P}}^{a} A_{\mathrm{P}}^{b}\right]=\frac{1}{2} \mathcal{T}\left[q_{i} \bar{q}_{j} \rightarrow A_{\mathrm{T}}^{a} A_{\mathrm{T}}^{b}\right]+\mathcal{O}\left(\frac{m}{E}\right)
$$

which demonstrates explicitly the TET (3.6).

\subsubsection{Pure gauge boson scattering amplitudes}

In this subsection, we study the 4-particle amplitudes of the pure gauge boson scattering processes $A_{\mathrm{P}}^{a} A_{\mathrm{P}}^{b} \rightarrow A_{\mathrm{P}}^{c} A_{\mathrm{P}}^{d}$ and $A_{\mathrm{T}}^{a} A_{\mathrm{T}}^{b} \rightarrow A_{\mathrm{T}}^{c} A_{\mathrm{T}}^{d}$ in the $3 \mathrm{~d}$ non-Abelian topologically massive YM (TMYM) theory, where the gauge field $A_{\mu}^{a}$ belongs to the adjoint representation of the $\mathrm{SU}(N)$ gauge group. The relevant Feynman diagrams are shown in figure 4.

We see that the 4-gauge boson scattering amplitudes $\mathcal{T}\left[A_{\mathrm{P}}^{a} A_{\mathrm{P}}^{b} \rightarrow A_{\mathrm{P}}^{c} A_{\mathrm{P}}^{d}\right] \equiv \mathcal{T}\left[4 A_{\mathrm{P}}^{a}\right]$ and $\mathcal{T}\left[A_{\mathrm{T}}^{a} A_{\mathrm{T}}^{b} \rightarrow A_{\mathrm{T}}^{c} A_{\mathrm{T}}^{d}\right] \equiv \mathcal{T}\left[4 A_{\mathrm{T}}^{a}\right]$ receive contributions from the contact diagram and the pole diagrams via $(s, t, u)$-channels. The kinematics of such 4-particle elastic scattering processes is defined in appendix A. Using the power counting rule (3.12), we find that for the scattering amplitude $\mathcal{T}\left[4 A_{\mathrm{P}}^{a}\right]$ the leading contributions of each diagram in figure 4 scale as $E^{4}$, while for the scattering amplitude $\mathcal{T}\left[4 A_{\mathrm{T}}^{a}\right]$ the individual leading contributions scale as $E^{0}$. Hence, using the TET identity (3.5) [or the TET (3.6)], we predict that the $A_{\mathrm{P}}^{a}$-amplitude should contain exact energy cancellations at the $\mathcal{O}\left(E^{4}\right), \mathcal{O}\left(E^{3}\right), \mathcal{O}\left(E^{2}\right)$, and $\mathcal{O}\left(E^{1}\right)$ respectively, because the leading energy-dependence of the $A_{\mathrm{P}}^{a}$-amplitude must match that of the corresponding $A_{\mathrm{T}}^{a}$-amplitude of $\mathcal{O}\left(E^{0}\right)$ on the r.h.s. of the TET identity (3.5) [or the TET (3.6)]. 

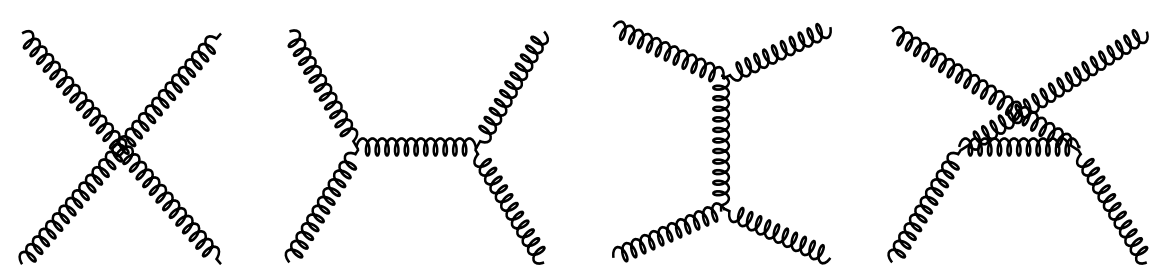

Figure 4. Feynman diagrams of the four-gauge boson scattering processes $A_{\mathrm{P}}^{a} A_{\mathrm{P}}^{b} \rightarrow A_{\mathrm{P}}^{c} A_{\mathrm{P}}^{d}$ and $A_{\mathrm{T}}^{a} A_{\mathrm{T}}^{b} \rightarrow A_{\mathrm{T}}^{c} A_{\mathrm{T}}^{d}$ via the contact interaction and $(s, t, u)$-channels in $3 \mathrm{~d}$ topologically massive YM theory.

Then, we compute explicitly the full scattering amplitude of $A_{\mathrm{P}}^{a}$ 's at tree level and present it in a compact form:

$$
\mathcal{T}\left[4 A_{\mathrm{P}}^{a}\right]=g^{2}\left(\frac{\mathcal{C}_{s} \mathcal{N}_{s}}{s-m^{2}}+\frac{\mathcal{C}_{t} \mathcal{N}_{t}}{t-m^{2}}+\frac{\mathcal{C}_{u} \mathcal{N}_{u}}{u-m^{2}}\right)
$$

where the color factors are defined as usual $\left(\mathcal{C}_{s}, \mathcal{C}_{t}, \mathcal{C}_{u}\right)=\left(C^{\text {abe }} C^{c d e}, C^{\text {ade }} C^{\text {bce }}, C^{\text {ace }} C^{\text {dbe }}\right)$ with $C^{a b c}$ denoting the structure constants of the gauge group. The numerators $\left(\mathcal{N}_{s}, \mathcal{N}_{t}, \mathcal{N}_{u}\right)$ take the following form:

$$
\begin{aligned}
\mathcal{N}_{s}= & \frac{4 m^{2}-s}{16 m^{3} s^{\frac{1}{2}}}\left[4 m s^{\frac{1}{2}}\left(5 m^{2}+4 s\right) c_{\theta}+\mathrm{i}\left(4 m^{4}+29 m^{2} s+3 s^{2}\right) s_{\theta}\right] \\
\mathcal{N}_{t}= & -\frac{c_{\theta / 2}}{16 m^{3}}\left(s^{\frac{1}{2}}+\mathrm{i} 2 m \tan \frac{\theta}{2}\right)^{2} \\
& \times\left\{4 m\left[13 m^{2}-3 s+\left(8 m^{2}-s\right) c_{\theta}\right] c_{\theta / 2}+\mathrm{i} s^{\frac{1}{2}}\left[22 m^{2}-3 s+\left(20 m^{2}-3 s\right) c_{\theta}\right] s_{\theta / 2}\right\} \\
\mathcal{N}_{u}= & \frac{s_{\theta / 2}}{16 m^{3}}\left(s^{\frac{1}{2}}-\mathrm{i} 2 m \cot \frac{\theta}{2}\right)^{2} \\
& \times\left\{4 m\left[13 m^{2}-3 s-\left(8 m^{2}-s\right) c_{\theta}\right] s_{\theta / 2}-\mathrm{i} s^{\frac{1}{2}}\left[22 m^{2}-3 s-\left(20 m^{2}-3 s\right) c_{\theta}\right] c_{\theta / 2}\right\}
\end{aligned}
$$

We note that in the $(2+1) \mathrm{d}$ spacetime there is a kinematic exchange symmetry between the scattering amplitudes of $t$-channel and $u$-channel, namely, their numerators obey the relation $\mathcal{N}_{u}(\pi+\theta)=-\mathcal{N}_{t}(\theta)$. We have verified that our numerators $(4.28 \mathrm{~b})-(4.28 \mathrm{c})$ indeed satisfy this kinematic exchange symmetry.

We note that each term on the r.h.s. of eq. (4.27) scales as $E^{3}$ at most because summing up each contribution of the contact diagram with the corresponding pole diagram already cancels $\mathcal{O}\left(E^{4}\right)$ terms, as we show in table 4 . For the high energy scattering with $E \gg m$, we expand the full amplitudes in terms of $1 / \bar{s}_{0}$, where $s_{0}=4 E^{2} \beta^{2}$ and $\bar{s}_{0}=4 \bar{E}^{2} \beta^{2}$ with $\bar{E}=E / m$. Thus, we can explicitly demonstrate the exact energy cancellations at each order of $E^{n}(n=4,3,2,1)$, respectively. We summarize our findings in table 4, from which we prove the following exact energy cancellations:

$$
\begin{aligned}
\mathcal{T}_{c j}^{(4)}+\mathcal{T}_{j}^{(4)} & =0, \\
\sum_{j}\left(\mathcal{T}_{c j}^{(3)}+\mathcal{T}_{j}^{(3)}\right) & =-\mathrm{i} 24 s_{\theta} \bar{s}_{0}^{\frac{3}{2}} c_{0}\left(\mathcal{C}_{s}+\mathcal{C}_{t}+\mathcal{C}_{u}\right)=0,
\end{aligned}
$$




\begin{tabular}{|c|c|c|c|c|}
\hline Amplitude & $\times \bar{s}_{0}^{2}$ & $\times \bar{s}_{0}^{3 / 2}$ & $\times \bar{s}_{0}$ & $\times \bar{s}_{0}^{1 / 2}$ \\
\hline $\mathcal{T}_{c s}$ & $8 s_{\theta} \mathcal{C}_{s}$ & $\mathrm{i} 32 s_{\theta} \mathcal{C}_{s}$ & $64 c_{\theta} \mathcal{C}_{s}$ & $\mathrm{i} 64 s_{\theta} \mathcal{C}_{s}$ \\
\hline $\mathcal{T}_{c t}$ & $-\left(5+4 c_{\theta}-c_{2 \theta}\right) \mathcal{C}_{t}$ & $-\mathrm{i} 8\left(2 s_{\theta}-s_{2 \theta}\right) \mathcal{C}_{t}$ & $-32\left(c_{\theta}-c_{2 \theta}\right) \mathcal{C}_{t}$ & $-\mathrm{i} 16\left(2 s_{\theta}-5 s_{2 \theta}\right) \mathcal{C}_{t}$ \\
\hline $\mathcal{T}_{c u}$ & $\left(5-4 c_{\theta}-c_{2 \theta}\right) \mathcal{C}_{u}$ & $-\mathrm{i} 8\left(2 s_{\theta}+s_{2 \theta}\right) \mathcal{C}_{u}$ & $-32\left(c_{\theta}+c_{2 \theta}\right) \mathcal{C}_{u}$ & $-\mathrm{i} 16\left(2 s_{\theta}+5 s_{2 \theta}\right) \mathcal{C}_{u}$ \\
\hline $\mathcal{T}_{s}$ & $-8 s_{\theta} \mathcal{C}_{s}$ & $-\mathrm{i} 56 s_{\theta} \mathcal{C}_{s}$ & $-192 c_{\theta} \mathcal{C}_{s}$ & $-\mathrm{i} 368 s_{\theta} \mathcal{C}_{s}$ \\
\hline $\mathcal{T}_{t}$ & $\left(5+4 c_{\theta}-c_{2 \theta}\right) \mathcal{C}_{t}$ & $-\mathrm{i} 8\left(s_{\theta}+s_{2 \theta}\right) \mathcal{C}_{t}$ & $-32\left(3 c_{\theta}+c_{2 \theta}\right) \mathcal{C}_{t}$ & $-\mathrm{i} 16\left(17 s_{\theta}+5 s_{2 \theta}\right) \mathcal{C}_{t}$ \\
\hline $\mathcal{T}_{u}$ & $-\left(5-4 c_{\theta}-c_{2 \theta}\right) \mathcal{C}_{u}$ & $-\mathrm{i} 8\left(s_{\theta}-s_{2 \theta}\right) \mathcal{C}_{u}$ & $-32\left(3 c_{\theta}-c_{2 \theta}\right) \mathcal{C}_{u}$ & $-\mathrm{i} 16\left(17 s_{\theta}-5 s_{2 \theta}\right) \mathcal{C}_{u}$ \\
\hline $\mathrm{Sum}$ & 0 & 0 & 0 & 0 \\
\hline
\end{tabular}

Table 4. Energy cancellations in the 4-gauge boson scattering amplitude of 3d non-Abelian TMYM theory, $\mathcal{T}\left[A_{\mathrm{P}}^{a} A_{\mathrm{P}}^{b} \rightarrow A_{\mathrm{P}}^{c} A_{\mathrm{P}}^{d}\right]=\mathcal{T}_{c}+\mathcal{T}_{s}+\mathcal{T}_{t}+\mathcal{T}_{u}$, where the amplitude from contact diagram is further decomposed into three sub-amplitudes according to their color factors, $\mathcal{T}_{c}=\mathcal{T}_{c s}+\mathcal{T}_{c t}+\mathcal{T}_{c u}$. The energy factor is defined as $\bar{s}_{0}=4 \bar{E}^{2} \beta^{2}$ and $\bar{E}=E / m$. A common overall factor $\left(g^{2} / 128\right)$ in each amplitude is not displayed for simplicity.

$$
\begin{aligned}
& \sum_{j}\left(\mathcal{T}_{c j}^{(2)}+\mathcal{T}_{j}^{(2)}\right)=-128 c_{\theta} \bar{s}_{0} c_{0}\left(\mathcal{C}_{s}+\mathcal{C}_{t}+\mathcal{C}_{u}\right)=0 \\
& \sum_{j}\left(\mathcal{T}_{c j}^{(1)}+\mathcal{T}_{j}^{(1)}\right)=-\mathrm{i} 304 s_{\theta} \bar{s}_{0}^{\frac{1}{2}} c_{0}\left(\mathcal{C}_{s}+\mathcal{C}_{t}+\mathcal{C}_{u}\right)=0
\end{aligned}
$$

where $j \in(s, t, u), c_{0}=g^{2} / 128$, and the superscript $(n)$ in the amplitudes $\left(\mathcal{T}_{c j}^{(n)}, \mathcal{T}_{j}^{(n)}\right)$ denotes the contributions at the $\mathcal{O}\left(E^{n}\right)$ with $n=1,2,3,4$. The amplitudes $\mathcal{T}_{c}$ (contributed by the contact diagram) and $\mathcal{T}_{j}$ (contributed by the gauge-boson-exchange in each channelj) are given by the sums:

$$
\mathcal{T}_{c}=\sum_{j, n} \mathcal{T}_{c j}^{(n)}, \quad \mathcal{T}_{j}=\sum_{n} \mathcal{T}_{j}^{(n)}
$$

where $j \in(s, t, u)$ and $n=4,3,2, \cdots$. From table 4 and eq. (4.29a), the $\mathcal{O}\left(E^{4}\right)$ contributions cancel exactly between the contact diagram and the pole diagrams in each channel of $j \in(s, t, u)$. Furthermore, it is striking to see that the sum of each $\mathcal{O}\left(E^{n}\right)$ contributions $(n=3,2,1)$ also cancel exactly because of the Jacobi identity $\mathcal{C}_{s}+\mathcal{C}_{t}+\mathcal{C}_{u}=0$, as shown in table 4 and eqs. (4.29b)-(4.29d).

After all these energy cancellations, we systematically derive the remaining scattering amplitude at $\mathcal{O}\left(E^{0}\right)$. We also compute the amplitude $\mathcal{T}\left[4 \tilde{A}_{\mathrm{T}}^{a}\right]$ which contains terms no more than $\mathcal{O}\left(E^{0}\right)$ by the direct power counting. Thus, we present both scattering amplitudes expanded to $\mathcal{O}\left(E^{0}\right)$ as follows:

$$
\begin{aligned}
& \mathcal{T}_{0}\left[4 A_{\mathrm{P}}^{a}\right]=g^{2}\left[\mathcal{C}_{s}\left(\frac{-9 c_{\theta}}{4}\right)+\mathcal{C}_{t}\left(\frac{-1-9 c_{\theta}-4 c_{2 \theta}}{4\left(1+c_{\theta}\right)}\right)+\mathcal{C}_{u}\left(\frac{1-9 c_{\theta}+4 c_{2 \theta}}{4\left(1-c_{\theta}\right)}\right)\right], \\
& \mathcal{T}_{0}\left[4 \tilde{A}_{\mathrm{T}}^{a}\right]=g^{2}\left[\mathcal{C}_{s}\left(\frac{-c_{\theta}}{4}\right)+\mathcal{C}_{t}\left(\frac{3-c_{\theta}}{4\left(1+c_{\theta}\right)}\right)+\mathcal{C}_{u}\left(\frac{-3-c_{\theta}}{4\left(1-c_{\theta}\right)}\right)\right],
\end{aligned}
$$

where we have denoted $\tilde{A}_{\mathrm{T}}^{a}=\frac{1}{\sqrt{2}} A_{\mathrm{T}}^{a}$ as before. Comparing the two amplitudes above, we find that they differ by an amount:

$$
\mathcal{T}_{0}\left[4 A_{\mathrm{P}}^{a}\right]-\mathcal{T}_{0}\left[4 \tilde{A}_{\mathrm{T}}^{a}\right]=-2 g^{2} c_{\theta}\left(\mathcal{C}_{s}+\mathcal{C}_{t}+\mathcal{C}_{u}\right)=0,
$$


which vanishes identically because of the Jacobi identity $\mathcal{C}_{s}+\mathcal{C}_{t}+\mathcal{C}_{u}=0$. This demonstrates explicitly the TET for the 4-gauge boson scattering in the non-Abelian TMYM theory:

$$
\mathcal{T}\left[A_{\mathrm{P}}^{a} A_{\mathrm{P}}^{b} \rightarrow A_{\mathrm{P}}^{c} A_{\mathrm{P}}^{d}\right]=\mathcal{T}\left[A_{\mathrm{T}}^{a} A_{\mathrm{T}}^{b} \rightarrow A_{\mathrm{T}}^{c} A_{\mathrm{T}}^{d}\right]+\mathcal{O}\left(\frac{m}{E}\right),
$$

which confirms the general TET (3.6) for the case of $N=4$.

To conclude this subsection, we stress that the present study has well understood and justified the structure of our gauge boson scattering amplitude (4.27) order by order under the high energy expansion, including the exact energy cancellations at each $\mathcal{O}\left(E^{n}\right)$ with $n=4,3,2,1$ and the proof of the TET relation (4.32) at $\mathcal{O}\left(E^{0}\right)$.

\subsection{Unitarity bounds on TMYM and TMG theories}

In this subsection, we analyze the partial wave amplitudes of the $3 \mathrm{~d}$ topologically massive gauge boson scattering and the $3 \mathrm{~d}$ topologically massive graviton scattering (section 5). We will demonstrate that the partial waves for either the topologically massive gauge boson scattering or the topologically massive graviton scattering have high energy behaviors no larger than $\mathcal{O}\left(E^{0}\right)$, so they can respect the perturbative unitarity bound.

For an $\mathrm{SU}(N)$ gauge theory, we define the gauge-singlet one-particle state:

$$
|0\rangle_{A_{\mathrm{P}}}=\frac{1}{\sqrt{2\left(N^{2}-1\right)}} \sum_{a=1}^{N^{2}-1}\left|A_{\mathrm{P}}^{a} A_{\mathrm{P}}^{a}\right\rangle .
$$

Thus, we can compute the scattering amplitude for the gauge-singlet state as follows:

$$
\mathcal{T}\left[|0\rangle_{A_{\mathrm{P}}} \rightarrow|0\rangle_{A_{\mathrm{P}}}\right]=\frac{g^{2} N}{2}\left(\frac{\mathcal{N}_{t}^{\prime}}{t-m^{2}}-\frac{\mathcal{N}_{u}^{\prime}}{u-m^{2}}\right) .
$$

In $d$-dimensions, the partial wave amplitude takes the following general form [53]:

$$
a_{\ell}(s)=\frac{s^{d / 2-2}}{C_{\ell}^{\nu}(1) \lambda_{d}} \int_{0}^{\pi} \mathrm{d} \theta\left[(\sin \theta)^{d-3} C_{\ell}^{\nu}(\cos \theta) \mathcal{T}_{\mathrm{el}}\right]
$$

where $C_{\ell}^{\nu}(x)$ is the Gegenbauer polynomial and

$$
\nu=\frac{1}{2}(d-3), \quad \lambda_{d}=2 \Gamma\left(\frac{1}{2} d-1\right)(16 \pi)^{d / 2-1} .
$$

The partial wave $a_{\ell}$ should satisfy the unitarity condition $\mathfrak{I m}\left(a_{\ell}\right) \geqslant\left|a_{\ell}\right|^{2}$, leading to [53-55]:

$$
\left|a_{\ell}\right| \leqslant 1, \quad\left|\mathfrak{R e}\left(a_{\ell}\right)\right| \leqslant \frac{1}{2}, \quad\left|\mathfrak{I m}\left(a_{\ell}\right)\right| \leqslant 1 .
$$

For the present study, we have $d=3$. Thus, we can compute the real part of the $s$-wave amplitude (4.36) as follows:

$$
\begin{aligned}
\mathfrak{R e}\left(a_{0}\right) & =\frac{1}{8 \pi \sqrt{s}} \int_{0}^{\pi} \mathrm{d} \theta \mathfrak{R e}\left(\mathcal{T}\left[|0\rangle_{A_{\mathrm{P}}} \rightarrow|0\rangle_{A_{\mathrm{P}}}\right]\right) \\
& =-\frac{g^{2} N\left(16 m^{4}+24 m^{2} s+s^{2}\right)}{32 \sqrt{s}\left(s-4 m^{2}\right)^{2}} \simeq-\frac{N g^{2}}{32 \sqrt{s}} .
\end{aligned}
$$


The imaginary part $\mathfrak{I m}\left(a_{0}\right)$ has collinear divergences around $\theta=0, \pi$ of the integral. After adding an angular cut on the scattering angle $(\delta \leqslant \theta \leqslant \pi-\delta)$ to remove the collinear divergences of the integral, we find that $\mathfrak{I m}\left(a_{0}\right)$ vanishes. Eq. (4.39) shows that for high energy scattering the leading partial wave amplitude $\mathfrak{R e}\left(a_{0}\right)$ scales as $E^{-1}$, which has good high energy behaviors. This is expected, because the $3 \mathrm{~d}$ TMYM theory is gauge-invariant and has a super-renormalizable gauge coupling $g$ with mass-dimension $+\frac{1}{2}$. Applying the unitarity condition (4.38) to $s$-wave amplitude (4.39), we derive the following perturbative unitarity bound:

$$
\sqrt{s} \geqslant \frac{g^{2} N}{16}
$$

which puts a lower limit on the scattering energy, in addition to the requirement of high energy expansion $\sqrt{s} \gg m$.

Next, we study the perturbative unitarity bound for the TMG theory in section 5 . Using the high-energy graviton scattering amplitude in eqs. (5.13)-(5.16), we compute the partial wave amplitudes of its real and imaginary parts as follows:

$$
\begin{aligned}
& \mathfrak{R e}\left(a_{0}\right) \simeq-\frac{15 \kappa^{2} m^{2}}{2048 \pi \sqrt{s}} \frac{(3 \cos \delta-\cos 3 \delta)}{\sin ^{3} \delta} \simeq-\frac{15 \kappa^{2} m^{2}}{1024 \pi \delta^{3} \sqrt{s}}, \\
& \mathfrak{I m}\left(a_{0}\right) \simeq-\frac{247 \kappa^{2} m}{49152 \pi} \frac{(3 \cos \delta-\cos 3 \delta)}{\sin ^{3} \delta} \simeq-\frac{247 \kappa^{2} m}{24576 \pi \delta^{3}},
\end{aligned}
$$

where we put an angular cut on the scattering angle $(\delta \leqslant \theta \leqslant \pi-\delta)$ to remove the collinear divergences of the integral. We see that both $\mathfrak{R e}\left(a_{0}\right)$ and $\mathfrak{I m}\left(a_{0}\right)$ exhibit good high-energy behaviors since they scale as $E^{-1}$ and $E^{0}$, respectively. Imposing the perturbative unitarity condition on the $s$-wave amplitude (4.41), we derive the unitarity bounds on the real and imaginary parts:

$$
\sqrt{s} \geqslant \frac{15 \kappa^{2} m^{2}}{1024 \pi \delta^{3}}, \quad m \leqslant \frac{49152 \pi \delta^{3}}{247 \kappa^{2}}
$$

where the first condition places a lower bound on the scattering energy proportional to $\kappa^{2} m^{2}$. The second condition puts an upper bound on the graviton mass $m$, proportional to $1 / \kappa^{2}$ which characterizes the ultraviolet cutoff scale of the TMG gravity since the $3 \mathrm{~d}$ gravitational coupling $\kappa^{2}=16 \pi G=2 / M_{\mathrm{Pl}}$ has a negative mass-dimension -1 , where $G$ and $M_{\mathrm{Pl}}$ denote the $3 \mathrm{~d}$ Newton constant and Planck mass respectively.

\section{Structure of topological graviton amplitude from double-copy}

In this section, we will study the extended double-copy construction of the massive graviton amplitude in the $3 \mathrm{~d}$ topologically massive gravity (TMG) from the massive gauge boson amplitude in the 3d TMYM theory. Our focus is to analyze the structure of massive graviton scattering amplitudes under high energy expansion and newly uncover a series of striking energy-cancellations of the graviton amplitudes in connection to the corresponding gauge boson amplitudes through the extended massive double-copy construction. These new findings were not covered by any previous literature. In section 5.1, starting from the $3 \mathrm{~d}$ 
action of the TMG we will analyze the equation of motion (EOM) of the $3 \mathrm{~d}$ graviton field and identify the physical polarization state of the graviton. Then, in section 5.2 we will extend the conventional double-copy method for massless gauge/gravity theories [17-19] to the $3 \mathrm{~d}$ topologically massive gauge/gravity theories. For this we will improve the original massive 4-gauge-boson scattering amplitude (4.27)-(4.28) by proper choice of the gauge transformation on its kinematic numerators. With these we can reconstruct the correct 4-graviton scattering amplitude in the TMG theory. We stress that a key feature of the $3 \mathrm{~d}$ TMYM and TMG theories is that these theories can realize the topological mass-generation of gauge bosons and gravitons in a fully gauge-invariant way under the path integral formulation, which is important for the successful double-copy construction in the $3 \mathrm{~d}$ massive gauge/gravity theories.

\section{$5.13 \mathrm{~d}$ topologically massive gravity}

In this subsection, we first introduce the $3 \mathrm{~d}$ action of the TMG. Then, we analyze the equation of motion of the $3 \mathrm{~d}$ graviton field and identify the physical polarization state of the graviton. We note that even though the $3 \mathrm{~d}$ massless Einstein gravity has no physical content $[1,2,56-60]$, including the gravitational Chern-Simons term can make the TMG fully nontrivial. The TMG action contains the conventional Einstein-Hilbert term and the gravitational Chern-Simons term [1,2]:

$$
S_{\mathrm{TMG}}=-\frac{2}{\kappa^{2}} \int \mathrm{d}^{3} x\left[\sqrt{-g} R-\frac{1}{2 \widetilde{m}} \varepsilon^{\mu \nu \rho} \Gamma_{\rho \beta}^{\alpha}\left(\partial_{\mu} \Gamma_{\alpha \nu}^{\beta}+\frac{2}{3} \Gamma_{\mu \gamma}^{\beta} \Gamma_{\nu \alpha}^{\gamma}\right)\right],
$$

where the $3 \mathrm{~d}$ gravitational coupling constant $\kappa$ is connected to the Planck mass $M_{\mathrm{Pl}}$ via $\kappa=2 / \sqrt{M_{\mathrm{Pl}}}$ with $M_{\mathrm{Pl}}=1 /(8 \pi G)$ and $G$ as the Newton constant. The parameter $\widetilde{m}$ in eq. (5.1) will provide the graviton mass $m=|\widetilde{m}|$, as shown in eq. (E.6). Under the weak field expansion $g_{\mu \nu}=\eta_{\mu \nu}+\kappa h_{\mu \nu}$ and the linearized diffeomorphism transformation $h_{\mu \nu} \rightarrow h_{\mu \nu}^{\prime}=h_{\mu \nu}+\partial_{\mu} \xi_{\nu}+\partial_{\nu} \xi_{\mu}$, the gravitational Chern-Simons term in eq. (5.1) changes into a total derivative, so the action is diffeomorphism invariant.

The nonlinear EOM is derived as follows [58-60]:

$$
R_{\mu \nu}-\frac{1}{2} g_{\mu \nu} R+\frac{1}{m} C_{\mu \nu}=0,
$$

where the Cotton tensor $C_{\mu \nu}=\varepsilon_{\mu}{ }^{\rho \sigma} \nabla_{\rho}\left(R_{\sigma \nu}-\frac{1}{4} g_{\sigma \nu} R\right)$ is symmetric and traceless. In eq. (5.2) and the discussions hereafter, we use the positive branch of the mass parameter $\tilde{m}>0$, which corresponds to the graviton with spin $\mathfrak{s}=+2[1,2]$. Then, we can expand the metric tensor around the Minkowski metric $g_{\mu \nu}=\eta_{\mu \nu}+\kappa h_{\mu \nu}$ and impose the transverse-traceless condition for the graviton field. With these, we obtain the linearized EOM from eq. (5.2):

$$
\left[\eta_{\mu \alpha} \eta_{\nu \beta}+\frac{1}{2 m}\left(\varepsilon_{\mu \rho \alpha} \eta_{\nu \beta}+\varepsilon_{\nu \rho \beta} \eta_{\mu \alpha}\right) \partial^{\rho}\right] \partial^{2} h_{\mathrm{P}}^{\alpha \beta}=0
$$

We may denote the operator in the square brackets of eq. (5.3) as

$$
\widehat{O}_{\mu \nu \alpha \beta}=\eta_{\mu \alpha} \eta_{\nu \beta}+\frac{1}{2 m}\left(\varepsilon_{\mu \rho \alpha} \eta_{\nu \beta}+\varepsilon_{\nu \rho \beta} \eta_{\mu \alpha}\right) \partial^{\rho} .
$$


Then, we act the operator $\widehat{O}_{\mu \nu \alpha \beta}$ twice on the graviton field and impose the transversetraceless condition on the physical graviton state, which leads to

$$
\left(\partial^{2}-m^{2}\right) \partial^{2} h_{\mathrm{P}}^{\mu \nu}=0 .
$$

This shows that the graviton field obeys a Klein-Gordon-like equation and carries the physical mass $m$.

Alternatively, we can "square" the EOM of the TMYM theory (2.5) and arrive at

$$
\begin{aligned}
& \left(\eta_{\mu \alpha} \eta_{\nu \beta}-\frac{\mathrm{i} \varepsilon_{\mu \rho \alpha} \eta_{\nu \beta} p^{\rho}}{m}-\frac{\mathrm{i} \varepsilon_{\nu \sigma \beta} \eta_{\mu \alpha} p^{\sigma}}{m}-\frac{\varepsilon_{\mu \rho \alpha} \varepsilon_{\nu \sigma \beta} p^{\rho} p^{\sigma}}{m^{2}}\right) \epsilon^{\alpha} \epsilon^{\beta} \\
& =2\left[\eta_{\mu \alpha} \eta_{\nu \beta}-\frac{\mathrm{i}}{2 m}\left(\varepsilon_{\mu \rho \alpha} \eta_{\nu \beta}+\varepsilon_{\nu \rho \beta} \eta_{\mu \alpha}\right) p^{\rho}\right] \epsilon^{\alpha \beta}=0,
\end{aligned}
$$

where in the second line we have used the relations:

$$
\begin{aligned}
& \epsilon_{\mu \nu}=\epsilon_{\mu} \epsilon_{\nu}, \quad p^{\mu} \epsilon_{\mu}=0, \quad \epsilon_{\mu}^{\mu}=0, \\
& \varepsilon_{\mu \rho \alpha} \varepsilon_{\nu \sigma \beta} p^{\rho} p^{\sigma}=\left(\eta_{\mu \beta} \eta_{\alpha \nu}-\eta_{\mu \nu} \eta_{\alpha \beta}\right) p^{2}+\eta_{\alpha \beta} p_{\mu} p_{\nu}+\eta_{\mu \nu} p_{\alpha} p_{\beta}-\eta_{\nu \alpha} p_{\mu} p_{\beta}-\eta_{\mu \beta} p_{\nu} p_{\alpha},
\end{aligned}
$$

with the momentum $p^{\mu}$ obeying the on-shell condition $p^{2}=-m^{2}$. Thus, we see that eq. (5.6) coincides with eq. (5.3) where the graviton field is expressed in the plane wave form $h_{\mathrm{P}}^{\mu \nu}=\epsilon_{\mathrm{P}}^{\mu \nu} e^{-\mathrm{i} p \cdot x}$, with $\epsilon_{\mathrm{P}}^{\mu \nu}=\epsilon_{\mathrm{P}}^{\mu} \epsilon_{\mathrm{P}}^{\nu}$. The graviton polarization tensor $\epsilon_{\mathrm{P}}^{\mu \nu}$ is the solution of the EOM (5.6), where the subscript " $\mathrm{P}$ " indicates that it corresponds to the physical polarization state of the graviton $h_{\mathrm{P}}^{\mu \nu}$. Then, we impose the following gauge-fixing term:

$$
\mathcal{L}_{\mathrm{GF}}=\frac{1}{\xi}\left(\mathcal{F}_{\mu}\right)^{2}, \quad \mathcal{F}^{\mu}=\partial_{\nu} h^{\mu \nu}-\frac{1}{2} \partial^{\mu} h
$$

With the above and making the weak field expansion, we can derive the graviton propagator, which we present in eq. (E.6) of appendix E.

\subsection{Double-copy construction of graviton amplitude and energy cancellations}

In this subsection, we extend the conventional double-copy method for massless gauge/gravity theories [17-19] and construct the massive 4-graviton scattering amplitude in the 3d TMG theory from the 4-gauge boson amplitude in the 3d TMYM theory.

We examine the kinematic numerators $\left\{\mathcal{N}_{j}\right\}$ of the original massive 4-gauge-boson scattering amplitude (4.27)-(4.28), and find that they violate the kinematic Jacobi identity due to $\sum_{j} \mathcal{N}_{j} \neq 0$. Then, we analyze the gauge boson amplitude (4.27) and find that it is invariant under the following gauge transformation:

$$
\mathcal{N}_{j} \rightarrow \mathcal{N}_{j}^{\prime}=\mathcal{N}_{j}+\Delta\left(s_{j}-m^{2}\right),
$$

where $j \in(s, t, u)$ and $\Delta$ is a coefficient. Thus, by requiring the gauge-transformed numerators $\left\{\mathcal{N}_{j}^{\prime}\right\}$ to satisfy the kinematic Jacobi identity $\sum_{j} \mathcal{N}_{j}^{\prime}=0$, we can determine the coefficient $\Delta$ as follows:

$$
\Delta=-\frac{\mathrm{i} \csc \theta}{32 m^{3}}\left[\left(16 m^{4} s^{-\frac{1}{2}}+8 m^{2} s^{\frac{1}{2}}-3 s^{\frac{3}{2}}\right)-\left(16 m^{4} s^{-\frac{1}{2}}-24 m^{2} s^{\frac{1}{2}}-3 s^{\frac{3}{2}}\right) c_{2 \theta}+\mathrm{i} 16 m s s_{2 \theta}\right] .
$$


Then, we apply the gauge transformation (5.9) to the numerators (4.28) and further derive the following new kinematic numerators $\left(\mathcal{N}_{s}^{\prime}, \mathcal{N}_{t}^{\prime}, \mathcal{N}_{u}^{\prime}\right)$ :

$$
\begin{aligned}
& \mathcal{N}_{s}^{\prime}=\frac{\mathrm{i} \csc \theta}{8 m s^{\frac{1}{2}}}\left[8 m^{4}+26 m^{2} s-7 s^{2}-\left(8 m^{4}+18 m^{2} s+s^{2}\right) c_{2 \theta}\right. \\
& \left.-\mathrm{i} m s^{\frac{1}{2}}\left(20 m^{2}+7 s\right) s_{2 \theta}\right], \\
& \mathcal{N}_{t}^{\prime}=-\frac{\mathrm{i} \csc \theta}{32 m s^{\frac{1}{2}}}\left[16 m^{4}+52 m^{2} s-14 s^{2}+\left(16 m^{4}+104 m^{2} s-15 s^{2}\right) c_{\theta}\right. \\
& -2\left(8 m^{4}+18 m^{2} s+s^{2}\right) c_{2 \theta}-\left(16 m^{4}+24 m^{2} s+s^{2}\right) c_{3 \theta} \\
& +\mathrm{i} m s^{\frac{1}{2}}\left(176 m^{2}-20 s\right) s_{\theta}-\mathrm{i} m s^{\frac{1}{2}}\left(40 m^{2}+14 s\right) s_{2 \theta} \\
& \left.-\mathrm{i} m s^{\frac{1}{2}}\left(32 m^{2}+8 s\right) s_{3 \theta}\right] \text {, } \\
& \mathcal{N}_{u}^{\prime}=-\frac{\mathrm{i} \csc \theta}{32 m s^{\frac{1}{2}}}\left[16 m^{4}+52 m^{2} s-14 s^{2}-\left(16 m^{4}+104 m^{2} s-15 s^{2}\right) c_{\theta}\right. \\
& -2\left(8 m^{4}+18 m^{2} s+s^{2}\right) c_{2 \theta}+\left(16 m^{4}+24 m^{2} s+s^{2}\right) c_{3 \theta} \\
& -\mathrm{i} m s^{\frac{1}{2}}\left(176 m^{2}-20 s\right) s_{\theta}-\mathrm{i} m s^{\frac{1}{2}}\left(40 m^{2}+14 s\right) s_{2 \theta} \\
& \left.+\mathrm{i} m s^{\frac{1}{2}}\left(32 m^{2}+8 s\right) s_{3 \theta}\right],
\end{aligned}
$$

which nicely obey the kinematic Jacobi identity $\sum_{j} \mathcal{N}_{j}^{\prime}=0$ by construction. With the above, we can reexpress the gauge boson amplitude (4.27) as follows:

$$
\mathcal{T}\left[4 A_{\mathrm{P}}^{a}\right]=g^{2}\left(\frac{\mathcal{C}_{s} \mathcal{N}_{s}^{\prime}}{s-m^{2}}+\frac{\mathcal{C}_{t} \mathcal{N}_{t}^{\prime}}{t-m^{2}}+\frac{\mathcal{C}_{u} \mathcal{N}_{u}^{\prime}}{u-m^{2}}\right)
$$

As a consistency check, we have also verified that the gauge-transformed numerators $(5.11 \mathrm{~b})-(5.11 \mathrm{c})$ satisfy the kinematic exchange symmetry between the $t$-channel and $u$-channel, namely, $\mathcal{N}_{u}^{\prime}(\pi+\theta)=-\mathcal{N}_{t}^{\prime}(\theta)$.

For the 4-particle scattering amplitudes of massive physical gravitons $h_{\mathrm{P}}=\epsilon_{\mathrm{P}}^{\mu \nu} h_{\mu \nu}$ in the $3 \mathrm{~d}$ TMG theory, we can use the power counting rule (3.13) or (3.14) of section 3.2 to count the energy-dependence of the graviton scattering amplitudes and find that the leading individual contributions to the tree-level amplitudes scale as $E^{12}$. But, using the extended double-copy approach for massive scattering amplitudes, we will prove that such leading contributions of $\mathcal{O}\left(E^{12}\right)$ in the 4-graviton scattering amplitudes must cancel down to $\mathcal{O}\left(E^{1}\right)$, namely, the substantial cancellations of $\mathcal{O}\left(E^{12}\right) \rightarrow \mathcal{O}\left(E^{1}\right)$ by an energy power of $E^{11}$.

For this purpose, we extend the conventional double-copy method with the colorkinematics duality for massless gauge/gravity theories [17-19] to the 3d topologically massive gauge/gravity theories. Using our improved massive-gauge-boson amplitude (5.12) of 3d TMYM theory and the color-kinematics duality $\mathcal{C}_{j} \rightarrow \mathcal{N}_{j}^{\prime}$, we construct the full scattering amplitude of physical gravitons, $\mathcal{M}\left[h_{\mathrm{P}} h_{\mathrm{P}} \rightarrow h_{\mathrm{P}} h_{\mathrm{P}}\right] \equiv \mathcal{M}\left[4 h_{\mathrm{P}}\right]$, in the $3 \mathrm{~d}$ TMG theory:

$$
\mathcal{M}\left[4 h_{\mathrm{P}}\right]=\frac{\kappa^{2}}{16}\left[\frac{\left(\mathcal{N}_{s}^{\prime}\right)^{2}}{s-m^{2}}+\frac{\left(\mathcal{N}_{t}^{\prime}\right)^{2}}{t-m^{2}}+\frac{\left(\mathcal{N}_{u}^{\prime}\right)^{2}}{u-m^{2}}\right],
$$


where we have made the gauge-gravity coupling conversion $g \rightarrow \kappa / 4$. (The same conversion factor will work for the $4 \mathrm{~d}$ double-copy of the massless gauge/gravity theories $[17,18]$ if the same normalization of color factors is adopted.) Then, substituting the improved kinematic numerators (5.11) into eq. (5.13), we derive the following compact formula for the 4-graviton scattering amplitude after significant simplifications:

$$
\mathcal{M}\left[4 h_{\mathrm{P}}\right]=-\frac{\kappa^{2} m^{2}\left(P_{0}+P_{2} c_{2 \theta}+P_{4} c_{4 \theta}+P_{6} c_{6 \theta}+\bar{P}_{2} s_{2 \theta}+\bar{P}_{4} s_{4 \theta}+\bar{P}_{6} s_{6 \theta}\right) \csc ^{2} \theta}{4096\left(3+\bar{s}_{0}\right)\left(4+\bar{s}_{0}\right)^{3 / 2}\left(2+\bar{s}_{0}-\bar{s}_{0} c_{\theta}\right)\left(2+\bar{s}_{0}+\bar{s}_{0} c_{\theta}\right)},
$$

where $\left(P_{j}, \bar{P}_{j}\right)$ are expressed as polynomial functions of the variable $\bar{s}_{0}=s_{0} / \mathrm{m}^{2}$,

$$
\begin{aligned}
& P_{0}=-4\left(7992 \bar{s}_{0}^{2}+4767 \bar{s}_{0}^{3}+692 \bar{s}_{0}^{4}\right)\left(4+\bar{s}_{0}\right)^{\frac{1}{2}}, \\
& P_{2}=\left(-221184-304128 \bar{s}_{0}-114048 \bar{s}_{0}^{2}-10928 \bar{s}_{0}^{3}+505 \bar{s}_{0}^{4}\right)\left(4+\bar{s}_{0}\right)^{\frac{1}{2}}, \\
& P_{4}=4\left(55296+45312 \bar{s}_{0}+13208 \bar{s}_{0}^{2}+1563 \bar{s}_{0}^{3}+58 \bar{s}_{0}^{4}\right)\left(4+\bar{s}_{0}\right)^{\frac{1}{2}}, \\
& P_{6}=-\left(98304+57344 \bar{s}_{0}+11264 \bar{s}_{0}^{2}+832 \bar{s}_{0}^{3}+17 \bar{s}_{0}^{4}\right)\left(4+\bar{s}_{0}\right)^{\frac{1}{2}}, \\
& \bar{P}_{2}=\mathrm{i}\left(-442368-663552 \bar{s}_{0}-300672 \bar{s}_{0}^{2}-46048 \bar{s}_{0}^{3}+540 \bar{s}_{0}^{4}+475 \bar{s}_{0}^{5}\right), \\
& \bar{P}_{4}=\mathrm{i} 4\left(110592+104448 \bar{s}_{0}+36880 \bar{s}_{0}^{2}+5828 \bar{s}_{0}^{3}+372 \bar{s}_{0}^{4}+5 \bar{s}_{0}^{5}\right), \\
& \bar{P}_{6}=-\mathrm{i}\left(196608+139264 \bar{s}_{0}+35328 \bar{s}_{0}^{2}+3776 \bar{s}_{0}^{3}+148 \bar{s}_{0}^{4}+\bar{s}_{0}^{5}\right) .
\end{aligned}
$$

Next, we make high energy expansion for the reconstructed graviton amplitude (5.14)(5.15) and derive the following leading order (LO) result:

$$
\mathcal{M}_{0}\left[4 h_{\mathrm{P}}\right]=-\frac{\mathrm{i} \kappa^{2}}{2048} m s_{0}^{\frac{1}{2}}\left(494 c_{\theta}+19 c_{3 \theta}-c_{5 \theta}\right) \csc ^{3} \theta
$$

It is striking to see that the above LO graviton amplitude actually scales as $\mathcal{O}(m E)$ under the high energy expansion. Because the direct application of our power counting rule (3.14) to the double-copy graviton amplitude (5.13) gives the scaling behavior $\mathcal{M}_{0}\left[4 h_{\mathrm{P}}\right]=\mathcal{O}\left(m^{-2} E^{4}\right)$, we can expect from eq. (5.16) the further nontrivial energy cancellations of $E^{4} \rightarrow E^{1}$, which we will discuss in the following paragraph.

Inspecting the expressions of $\left(\mathcal{N}_{j}, \mathcal{N}_{j}^{\prime}\right)$ in eqs. (4.28) and (5.11), we find that they contain individual leading terms scaling as $\left(E^{5}, E^{3}\right)$, respectively. This shows that the gauge transformation (5.9) leads to an energy cancellation of $E^{5} \rightarrow E^{3}$ in each of the transformed numerators $\mathcal{N}_{j}^{\prime}$. This has an important impact on the energy dependence of the double-copy amplitude (5.13), namely, in each channel of $\mathcal{N}_{j}^{\prime 2} /\left(s_{j}-m^{2}\right)$, it contains a leading energy term behaving as $E^{4}$, rather than $E^{8}$ from $\mathcal{N}_{j}^{2} /\left(s_{j}-m^{2}\right)$. In comparison with the leading energy-dependence of the individual diagrams contributing to the tree-level 4-graviton amplitude which scales as $E^{12}$ by the direct power counting rule (3.13)-(3.14), our double-copy construction (5.13) demonstrates that the graviton amplitude could have leading energy dependence of $E^{4}$ in each channel. Hence, this double-copy construction guarantees a nontrivial large energy cancellation in the original four graviton scattering amplitude: $E^{12} \rightarrow E^{4}$, which cancels the leading energy dependence by a large power factor of $E^{8}$. 


\begin{tabular}{|c|c|c|c|}
\hline Amplitude & $\times \bar{s}_{0}^{2}$ & $\times \bar{s}_{0}^{3 / 2}$ & $\times \bar{s}_{0}$ \\
\hline $\mathcal{M}_{s}$ & $-\frac{99+28 c_{2 \theta}+c_{4 \theta}}{1-c_{2 \theta}}$ & $-\mathrm{i} 14\left(15 c_{\theta}+c_{3 \theta}\right) \csc \theta$ & $-\frac{2\left(75+326 c_{2 \theta}+47 c_{4 \theta}\right)}{1-c_{2 \theta}}$ \\
\hline $\mathcal{M}_{t}$ & $\frac{99+28 c_{2 \theta}+c_{4 \theta}}{4\left(1-c_{\theta}\right)}$ & $\mathrm{i}\left(102+105 c_{\theta}+70 c_{2 \theta}+7 c_{3 \theta}+4 c_{4 \theta}\right) \csc \theta$ & $\frac{75-107 c_{\theta}+326 c_{2 \theta}+268 c_{3 \theta}+47 c_{4 \theta}+31 c_{5 \theta}}{1-c_{2 \theta}}$ \\
\hline $\mathcal{M}_{u}$ & $\frac{99+28 c_{2 \theta}+c_{4 \theta}}{4\left(1+c_{\theta}\right)}$ & $\mathrm{i}\left(-102+105 c_{\theta}-70 c_{2 \theta}+7 c_{3 \theta}-4 c_{4 \theta}\right) \csc \theta$ & $\frac{75+107 c_{\theta}+326 c_{2 \theta}-268 c_{3 \theta}+47 c_{4 \theta}-31 c_{5 \theta}}{1-c_{2 \theta}}$ \\
\hline Sum & 0 & 0 & 0 \\
\hline
\end{tabular}

Table 5. Exact energy cancellations at each order of $E^{4}, E^{3}$, and $E^{2}$ in our 4-graviton scattering amplitude (5.13) by double-copy construction. Here the notations $\left(s_{0}, \bar{s}_{0}\right)$ are defined by $\bar{s}_{0}=s_{0} / m^{2}$ and $s_{0}=4 E^{2} \beta^{2}$. A common overall factor $\left(\kappa^{2} m^{2} / 1024\right)$ in each amplitude is not displayed for simplicity.

Furthermore, it is striking to see that our summed full amplitude (5.13) actually scales as $E^{1}$ under high energy expansion as shown in the above eq. (5.16). We can demonstrate explicitly this large energy cancellation of $E^{4} \rightarrow E^{1}$, which includes exact cancellations of the energy power terms at each order of $E^{4}, E^{3}$, and $E^{2}$. We summarize our findings on these exact energy cancellations of the full amplitude (5.13) into table 5. We may understand the reason for such energy cancellations of $E^{4} \rightarrow E^{1}$ as follows. We first note that an $S$-matrix element $\mathbb{S}$ with $\mathcal{E}$ external states and $L$ loops $(L \geqslant 0)$ in the $(2+1)$ d spacetime has mass-dimension $D_{\mathbb{S}}=3-\frac{1}{2} \mathcal{E}$ as given by eq. (3.7). Thus, the 4 graviton amplitude $\mathcal{M}\left[4 h_{\mathrm{P}}\right]$ has mass-dimension $D_{\mathcal{M}}=1$. At tree level it contains the gravitational coupling $\kappa^{2}$ of mass-dimension -1 . Hence, we can express the 4-graviton amplitude $\mathcal{M}\left[4 h_{\mathrm{P}}\right]=\kappa^{2} \overline{\mathcal{M}}\left[4 h_{\mathrm{P}}\right]$, where $\overline{\mathcal{M}}\left[4 h_{\mathrm{P}}\right]$ has mass-dimension equal +2 . The treelevel amplitude $\overline{\mathcal{M}}\left[4 h_{\mathrm{P}}\right]$ contains only two parameters $(E, m)$, each of which has massdimension +1 . With these we can deduce the scaling behavior $\overline{\mathcal{M}}\left[4 h_{\mathrm{P}}\right] \propto m^{n_{1}} E^{n_{2}}$ with $n_{1}+n_{2}=2$, under high energy expansion. Hence, for the energy terms of $E^{n_{2}}$ with $n_{2}=4,3,2$, we deduce the mass-power factor $n_{1}=-2,-1,0$, respectively. This means that in the massless limit $m \rightarrow 0$, the physical graviton amplitude $\overline{\mathcal{M}}\left[4 h_{\mathrm{P}}\right]$ would go to infinity (for $n_{2} \geqslant 3$ ) or remain constant (for $n_{2}=2$ ). But, in the $m \rightarrow 0$ limit, the $3 \mathrm{~d}$ graviton field becomes unphysical and has no physical degree of freedom [58-60]; so the amplitude $\overline{\mathcal{M}}\left[4 h_{\mathrm{P}}\right]$ should vanish because the physical graviton $h_{\mathrm{P}}$ no longer exists in the massless limit. This means that the $m^{n_{1}} E^{n_{2}}$ terms with $n_{1}=-2,-1,0$ should vanish, and the physical amplitude $\overline{\mathcal{M}}\left[4 h_{\mathrm{P}}\right]$ has to start with the leading behavior of $m^{1} E^{1}$ under high energy expansion. Thus, it is expected that the energy cancellations should hold at each order of $E^{4}, E^{3}$, and $E^{2}$, in agreement with what we first uncovered by explicit calculations in table 5 .

In summary, our double-copied graviton amplitudes in eqs. (5.13)-(5.16) and table 5 explicitly demonstrate a new type of large energy cancellations in the original 4-graviton amplitude at tree level for the 3d TMG theory:

$$
\mathcal{O}\left(E^{12}\right) \longrightarrow \mathcal{O}\left(E^{1}\right), \quad\left(\text { for } \mathcal{E}_{h_{\mathrm{P}}}=4 \text { in } 3 \mathrm{~d} \text { TMG }\right) .
$$

With this extended double-copy construction, we have established a new correspondence between the two types of leading energy cancellations in the massive scattering amplitudes: 
$E^{4} \rightarrow E^{0}$ in the TMYM theory and $E^{12} \rightarrow E^{1}$ in the TMG theory. We also note that in eq. (5.17) the exact energy cancellations in the 4-graviton amplitude down by a large power of $E^{11}$ are even much more severe than the energy cancellations $\left(E^{10} \rightarrow E^{2}\right)$ in the massive 4-longitudinal KK graviton scattering amplitudes of the compactified $5 \mathrm{~d}$ KK Einstein gravity found before by explicit calculations [61-63] and by the double-copy construction [27].

In passing, during the finalization of the present paper in this summer, we became aware of a recent new paper [64] which directly calculated the graviton amplitude of the 3d TMG with very lengthy expressions in its eq. (C.1) where all the polarization tensors (vectors) take symbolic forms. We have quantitatively compared our full graviton amplitude (5.13) (by double-copy construction) with their eq. (C.1) and find agreement after substituting explicitly all the polarization formulas into eq. (C.1) and making substantial simplifications of eq. (C.1). This comparison gives an independent verification of our double-copy result. Our work has fully different focus from [64] and our analyses differ from [64] in several essential ways. (i). The main part of our work (sections 2-4) is to analyze the mechanism of topological mass-generation of gauge bosons and uncover nontrivial energy cancellations in the gauge boson scattering amplitudes. These were not studied by [64]. (ii).For this purpose, we newly formulated the $3 d$ topological mass-generation mechanism at $S$-matrix level, and newly proposed and proved the TET for $N$-point gauge boson amplitudes in section 3. We further verified the TET explicitly by computing the four-point scattering amplitudes of various high-energy processes for both Abelian and non-Abelian gauge theories (with and without matter fields) in sections 4.1-4.2. These were not considered by [64]. (iii).Our whole study on the gauge boson amplitudes and the double-copied graviton amplitudes has focused on the structure of the scattering amplitudes under high energy expansion and on the mechanism of nontrivial large energy cancellations as summarized in tables 1-5 and eq. (5.17). For this, we newly constructed the general $3 \mathrm{~d}$ power counting method in section 3.2, and used it together with the TET to prove the nontrivial energy cancellations for $N$-point gauge boson amplitudes in section 3.3. These were not covered by [64]. (iv).We also note that eq. (C.2) of [64] further gave more compact formula of the 4-graviton amplitude in a very different Briet coordinate system and cannot be directly compared to our double-copied graviton amplitude (5.14)-(5.15). Their 4-gauge boson amplitude in eq. (C.3) was also given in the Briet coordinate system and cannot be directly compared to our eq. (5.11)-(5.12). The eqs. (4.12)-(4.13) of [64] gave 4-gauge boson amplitude with all polarization vectors in symbolic forms. We have further confirmed with the authors of [64] that our gauge boson amplitude (4.27) and their eq. (4.13) are in good agreement after substituting all the polarization formulas into their eq. (4.13) and after taking into account the notational difference in defining the Mandelstam variables. ${ }^{5}$ We stress that the parts of our study for the four-gauge boson amplitudes and double-copied four-graviton amplitudes have focused on analyzing their structures of energy-dependence and on uncovering the striking large energy cancellations in these amplitudes as well as

\footnotetext{
${ }^{5}$ After posting this paper to arXiv:2110.05399, we had helpful discussions with the authors of ref. [64]. We thank them for the comparison between their eq. (4.13) and our eq. (4.28) which confirms the good agreement between the independent analyses on both sides.
} 
the mechanism of such energy cancellations. These new findings were not covered by [64] whose independent study had fully different focus. We also note that the structures of our non-Abelian gauge boson amplitudes (4.27) and (5.12) are well understood and justified by nontrivial self-consistency checks as we explained at the end of section 4.2 .2 and showed in tables $4-5$.

\section{Conclusions}

Understanding the mechanism of topological mass-generation and the structure of the scattering amplitude in the 3d Chern-Simons (CS) gauge and gravity theories is important for applying the modern quantum field theories to particle physics and condensed matter physics [1-4]. In 3d spacetime the existence of the CS actions for gauge bosons and gravitons is theoretically unavoidable and compelling. This generates gauge-invariant topological mass-terms for gauge bosons and gravitons without invoking the conventional Higgs mechanism [6-10] and leads to good high energy behaviors for the scattering amplitudes of topologically massive gauge bosons and gravitons.

In this work, we systematically studied the mechanism of the topological massgenerations in $3 \mathrm{~d}$ CS gauge theories and formulated it at the $S$-matrix level. For this, we proposed and proved a new Topological Equivalence Theorem (TET) for understanding the structure of the scattering amplitudes of physical gauge bosons $\left(A_{\mathrm{P}}^{a}\right)$ in the topologically massive gauge theories. We newly uncovered the nontrivial large energy cancellations in the $N$-point gauge boson scattering amplitudes for both the Abelian and non-Abelian CS gauge theories. We further used an extended double-copy approach to analyze the structure of the graviton scattering amplitudes in the $3 \mathrm{~d}$ topologically massive gravity (TMG) theory, with which we reconstructed the physical graviton scattering amplitudes from that of the corresponding physical gauge bosons. From these, we newly uncovered a series of striking large energy cancellations in the four-point physical graviton scattering amplitudes, which ensure such massive scattering amplitudes to have good high energy behaviors and obey the perturbative unitarity bounds. We summarize these findings more explicitly as follows.

In section 2, we analyzed the dynamics of topological mass-generation in the $3 \mathrm{~d}$ CS gauge theories. In such dynamics, including the CS term (2.1) automatically converts the gauge boson's transverse polarization state $A_{\mathrm{T}}^{a}$ (combined with its longitudinal polarization state $\left.A_{\mathrm{L}}^{a}\right)$ into the massive physical polarization state $A_{\mathrm{P}}^{a} \propto\left(A_{\mathrm{T}}^{a}+A_{\mathrm{L}}^{a}\right)$ as given in eq. (2.12), while making its orthogonal combination $A_{\mathrm{X}}^{a} \propto\left(A_{\mathrm{T}}^{a}-A_{\mathrm{L}}^{a}\right)$ in eq. (2.13a) become an unphysical state. This topological mass-generation mechanism has essential difference from the conventional Higgs mechanism [6-10], because the CS term generates gauge-invariant mass term of $A_{\mu}^{a}$ and no spontaneous gauge symmetry breaking and Higgs boson are invoked.

In section 3, we newly proposed and proved the TET to formulate the topological mass-generation of gauge bosons at the $S$-matrix level, which quantitatively connects the $N$-point scattering amplitudes of physical gauge bosons $A_{\mathrm{P}}^{a}$ to the amplitudes of the corresponding transverse gauge boson states $A_{\mathrm{T}}^{a}$ in the high energy limit. For this, we established the general TET identity (3.5), with which we derived the TET (3.6) under high energy expansion. We presented a new energy power counting rule (3.12) to count the leading energy 
dependence of the $N$-point scattering amplitudes for the 3 d topologically massive gauge theories and another power counting rule (3.13) to count the leading energy dependence of the $N$-point scattering amplitudes for the $3 \mathrm{~d}$ topologically massive gravity (TMG) theory. (A generalized power counting method in $d$-dimensions is given in appendix B.) With these, we demonstrated that our TET identity (3.5) provides a general mechanism of nontrivial energy cancellations in the $N$-point $A_{\mathrm{P}}^{a}$-amplitudes because the net energy dependence of a given $N$-point $A_{\mathrm{P}}^{a}$-amplitude must match that of the leading $A_{\mathrm{T}}^{a}$-amplitude on the r.h.s. of eq. (3.5a). For the high-energy scattering of $N$-gauge bosons $A_{\mathrm{P}}^{a}$ (with $N \geqslant 4$ ), the TET identity (3.5) [or TET (3.6)] guarantees the nontrivial large energy cancellations in the $A_{\mathrm{P}}^{a}$-amplitude: $E^{4} \rightarrow E^{4-N}$.

In section 4, we explicitly demonstrated the TET (3.6) for the first time by using various high-energy four-particle scattering amplitudes in both the Abelian and non-Abelian topologically massive CS gauge theories. In sections 4.1.1-4.1.2, we computed the scattering amplitudes of the annihilation processes $\phi^{-} \phi^{+} \rightarrow A_{\mathrm{P}} A_{\mathrm{P}}\left(\phi^{-} \phi^{+} \rightarrow A_{\mathrm{T}} A_{\mathrm{T}}\right)$ and Compton scattering $\phi^{-} A_{\mathrm{P}} \rightarrow \phi^{-} A_{\mathrm{P}}\left(\phi^{-} A_{\mathrm{T}} \rightarrow \phi^{-} A_{\mathrm{T}}\right)$ in the topologically massive scalar QED (TMSQED), as shown in figure 1. In parallel, we computed the scattering amplitudes of annihilation processes $e^{-} e^{+} \rightarrow A_{\mathrm{P}} A_{\mathrm{P}}\left(e^{-} e^{+} \rightarrow A_{\mathrm{T}} A_{\mathrm{T}}\right)$ and Compton scattering $e^{-} A_{\mathrm{P}} \rightarrow e^{-} A_{\mathrm{P}}\left(e^{-} A_{\mathrm{T}} \rightarrow e^{-} A_{\mathrm{T}}\right)$ in the topologically massive spinor QED (TMQED), as shown in figure 2 . From these analyses, we newly uncovered the nontrivial energy cancellations of $E^{2} \rightarrow E^{0}$ in each $A_{\mathrm{P}}$-amplitude, which are summarized in tables 1-2 and in eqs. (4.3)(4.6) and eqs. (4.11a)-(4.11b). We further computed the remaining nonzero scattering amplitudes of $\mathcal{O}\left(E^{0}\right)$ and proved explicitly the validity of the TET as in eqs. (4.8) and (4.13).

Next, in section 4.2 we studied the structure of scattering amplitudes in the nonAbelian topologically massive QCD (TMQCD). In section 4.2.1, we computed the quarkantiquark annihilation processes $q_{i} \bar{q}_{j} \rightarrow A_{\mathrm{P}}^{a} A_{\mathrm{P}}^{b}\left(q_{i} \bar{q}_{j} \rightarrow A_{\mathrm{T}}^{a} A_{\mathrm{T}}^{b}\right)$ for the TMQCD, which are shown in figure 3 and contain additional $s$-channel diagram induced by the non-Abelian cubic vertex. We uncovered nontrivial energy cancellations of $E^{2} \rightarrow E^{0}$ in the $A_{\mathrm{P}}^{a}$-amplitude as summarized by table 3 and eq. (4.21). We further computed the remaining nonzero $A_{\mathrm{P}^{-}}^{a}$ amplitude and $A_{\mathrm{T}}^{a}$-amplitude of $\mathcal{O}\left(E^{0}\right)$, and proved explicitly that the TET holds for the TMQCD as in eq. (4.26). Then, in section 4.2.2, we systematically analyzed the 4-gauge boson scattering amplitudes of $A_{\mathrm{P}}^{a} A_{\mathrm{P}}^{b} \rightarrow A_{\mathrm{P}}^{c} A_{\mathrm{P}}^{d}$ and $A_{\mathrm{T}}^{a} A_{\mathrm{T}}^{b} \rightarrow A_{\mathrm{T}}^{c} A_{\mathrm{T}}^{d}$ in the TMQCD, which are shown in figure 4 . We newly uncovered the nontrivial large energy cancellations of $E^{4} \rightarrow E^{0}$ in the $A_{\mathrm{P}}^{a}$-amplitude, at each order of $\left(E^{4}, E^{3}, E^{2}, E^{1}\right)$ respectively, which are summarized in table 4 and eqs. (4.29a)-(4.29d). We further computed the remaining nonzero $A_{\mathrm{P}}^{a}$-amplitude and $A_{\mathrm{T}}^{a}$-amplitude at $\mathcal{O}\left(E^{0}\right)$ as given in eqs. (4.31a)-(4.31b). With these and the Jacobi identity, we proved explicitly that the TET indeed holds for the 4-gauge boson scattering amplitudes of the TMQCD, as shown in eq. (4.33). Finally, in section 4.3, we analyzed the perturbative unitarity of the TMYM and TMG theories. We found that the partial wave amplitudes (4.39) and (4.41) exhibit good high energy behaviors as they scale as $E^{-1}$ or $E^{0}$ in the high energy limit. This is expected for the $3 \mathrm{~d}$ TMYM theory since its gauge coupling has mass-dimension $+\frac{1}{2}$ and thus is super-renormalizable. This issue becomes much more nontrivial for the $3 \mathrm{~d}$ TMG theory since its Newton constant 
$G \propto \kappa^{2}$ has mass-dimension -1 . But we should expect this theory to exhibit good high energy behavior because the massive physical graviton scattering amplitudes in the TMG theory can be reconstructed from the corresponding massive gauge boson amplitudes in the TMYM theory via extended double-copy approach, as shown in section 5 .

In section 5, we extended the conventional double-copy approach to reconstruct the massive 4-graviton scattering amplitude of the $3 \mathrm{~d}$ TMG theory by using the massive 4-gauge boson amplitude of the 3d TMYM theory. We found that the reconstructed tree-level 4graviton scattering amplitude scales as $E^{1}$ under high energy expansion. We made the gauge transformation (5.9) on the kinematic numerators $\mathcal{N}_{j}$ of eq. (4.28) in the 4-gauge boson scattering amplitude (4.27) such that the new numerators $\mathcal{N}_{j}^{\prime}$ in eq. (5.11) obey the Jacobi identity. The gauge transformation of $\mathcal{N}_{j} \rightarrow \mathcal{N}_{j}^{\prime}$ leads to the energy cancellations of $E^{5} \rightarrow E^{3}$ in each kinematic numerator. This determines the individual leading energy dependence of the reconstructed graviton amplitude (5.13) to be no more than $\mathcal{O}\left(E^{4}\right)$. By explicit computations, we further uncovered new energy cancellations of $E^{4} \rightarrow E^{1}$ in the graviton scattering amplitude (5.13) as summarized in table 5. Then, we computed the remaining nonzero graviton amplitude as in eq. (5.16) which scales as $\mathcal{O}\left(E^{1}\right)$. In contrast, applying the power counting rule (3.14) we found that the individual contributions to the 4-graviton amplitude have leading energy dependence behave as $E^{12}$. From these together, we demonstrated a new type of striking energy cancellations in the 4-graviton scattering amplitude as in eq. (5.17), $\mathcal{O}\left(E^{12}\right) \rightarrow \mathcal{O}\left(E^{1}\right)$, for the $3 \mathrm{~d}$ TMG theory. Furthermore, with the extended double-copy construction, we established a new correspondence between the two types of leading energy cancellations in the massive scattering amplitudes: $E^{4} \rightarrow E^{0}$ in the TMYM theory and $E^{12} \rightarrow E^{1}$ in the TMG theory.

Our present findings are highly nontrivial and encouraging. We already generally proved our TET for the $N$-point gauge boson scattering amplitudes (section 3.1 ) and uncovered the mechanism of large energy cancellations for these $N$-point amplitudes by using the TET and the general energy power counting method in 3d (sections 3.2-3.3). It would be also interesting to extend our current explicit calculations and analyses to the $N$-point scattering amplitudes of gauge bosons (gravitons) in the TMYM (TMG) theories with more external states (such as $N=5$ and $N=6$ ). Since the 3 d topologically massive CS gauge theories are super-renormalizable and have good high energy behaviors, it would be desirable to extend the present tree-level analyses up to loop levels. We will pursue such extended studies in future works.

\section{Acknowledgments}

We thank Stanley Deser and Henry Tye for useful discussions. We also thank Nathan Moynihan and Mariana Carrillo-González for useful discussions. This research was supported in part by National NSF of China (under grants Nos.11835005, 12175136), by National Key R\&D Program of China (under grant No.2017YFA0402204), and by the CAS Center for Excellence in Particle Physics (CCEPP). 


\section{A Kinematics and Feynman rules of 3d CS gauge theories}

In this appendix, we present definitions of relevant kinematic variables for the 4-particle scattering process and the Feynman rules for the $3 \mathrm{~d}$ topologically massive Chern-Simons gauge theories.

For the present analysis, we choose the following metric signature and the rank-3 anti-symmetric tensor:

$$
\eta_{\mu \nu}=\eta^{\mu \nu}=\operatorname{diag}(-1,1,1), \quad \varepsilon^{012}=-\varepsilon_{012}=1 .
$$

Thus, we have the momentum on-shell condition $p^{2}=-m^{2}$.

For the $2 \rightarrow 2$ elastic scattering process, the momenta in the center-of-mass frame can be defined as follows:

$$
\begin{array}{ll}
p_{1}^{\mu}=E(1,0, \beta), & p_{2}^{\mu}=E(1,0,-\beta), \\
p_{3}^{\mu}=E\left(1, \beta s_{\theta}, \beta c_{\theta}\right), & p_{4}^{\mu}=E\left(1,-\beta s_{\theta},-\beta c_{\theta}\right),
\end{array}
$$

where we have defined $\beta=\sqrt{1-m^{2} / E^{2}}$. Thus, we further define the Mandelstam variables:

$$
\begin{aligned}
& s=-\left(p_{1}+p_{2}\right)^{2}=4 E^{2}, \\
& t=-\left(p_{1}-p_{4}\right)^{2}=-\frac{1}{2} s \beta^{2}\left(1+c_{\theta}\right), \\
& u=-\left(p_{1}-p_{3}\right)^{2}=-\frac{1}{2} s \beta^{2}\left(1-c_{\theta}\right) .
\end{aligned}
$$

For convenience of our analysis, we can use the relation $E^{2}=E^{2} \beta^{2}+m^{2}$ to define another set of Mandelstam variables $\left(s_{0}, t_{0}, u_{0}\right)$ :

$$
s_{0}=4 E^{2} \beta^{2}, \quad t_{0}=-\frac{1}{2} s_{0}\left(1+c_{\theta}\right), \quad u_{0}=-\frac{1}{2} s_{0}\left(1-c_{\theta}\right) .
$$

The summations of $(s, t, u)$ and $\left(s_{0}, t_{0}, u_{0}\right)$ obey the conditions:

$$
s+t+u=4 m^{2}, \quad s_{0}+t_{0}+u_{0}=0 .
$$

In the rest frame with 3 -momentum $p^{\mu}=(m, 0,0) \equiv \bar{p}^{\mu}$, we can solve eq. (2.5) and derive the polarization vector:

$$
\epsilon^{\mu}(\bar{p})=\frac{1}{\sqrt{2}}(0,1,-\mathrm{i} \mathfrak{s})
$$

where $\mathfrak{s}=\tilde{m} / m= \pm 1$ and $m=|\widetilde{m}|$. We note that the in the rest frame the gauge boson polarization vector has zero time-component and its two possible forms are not independent due to the relation $\epsilon_{2}^{\mu}=i \mathfrak{s} \epsilon_{1}^{\mu}$. Furthermore, by choosing the orthonormal basis $e_{1}^{j}=(1,0)$ and $e_{2}^{j}=(0,1)$ in a plane, we can define a polarization basis:

$$
e_{ \pm}^{j}=\frac{1}{\sqrt{2}}\left(e_{1}^{j} \pm \mathrm{i} e_{2}^{j}\right)=\frac{1}{\sqrt{2}}(1, \pm \mathrm{i}) .
$$


Thus, in the rest frame the spatial part of the polarization vector $\epsilon^{\mu}(\bar{p})$ can be decomposed in terms of the basis $\left\{e_{ \pm}^{j}\right\}$ :

$$
\epsilon^{j}(\bar{p})=\epsilon_{+} e_{+}^{j}+\epsilon_{-} e_{-}^{j},
$$

where the coefficients $\left(\epsilon_{+}, \epsilon_{-}\right)$satisfy $\left(\epsilon_{+}, \epsilon_{-}\right)=(0,1)$ for $\mathfrak{s}=+1$ and $\left(\epsilon_{+}, \epsilon_{-}\right)=(1,0)$ for $\mathfrak{s}=-1$ [38]. So, it is clear that in the 3 d Chern-Simons gauge theory, the case of $\mathfrak{s}=+1$ (or, $\mathfrak{s}=-1$ ) only allows one physical polarization state $\epsilon_{-}\left(\right.$or, $\left.\epsilon_{+}\right)$of the gauge boson, as expected.

After taking the Lorentz boost along an arbitrary direction, the gauge boson momentum can be generally written as $p^{\mu}=E\left(1, \beta s_{\theta}, \beta c_{\theta}\right)$. Thus, we can Lorentz-boost the rest-frame polarization vector (A.6) to the following general polarization vector:

$$
\epsilon_{\mathrm{P}}^{\mu}(p)=\frac{1}{\sqrt{2}}\left(\frac{\mathrm{i} p_{1}+\mathfrak{s} p_{2}}{m}, \mathrm{i}+\frac{p_{1}\left(\mathrm{i} p_{1}+\mathfrak{s} p_{2}\right)}{m\left(m-p_{0}\right)}, \mathfrak{s}+\frac{p_{2}\left(\mathrm{i} p_{1}+\mathfrak{s} p_{2}\right)}{m\left(m-p_{0}\right)}\right),
$$

where $\epsilon_{+}^{\mu}=-\left(\epsilon_{-}^{\mu}\right)^{*}$. Thus, by substituting eq. (A.2) into eq. (A.9), we derive the following explicit formulas of the physical polarization vectors:

$$
\begin{aligned}
\epsilon_{1}^{\mu} & =\frac{\mathfrak{s}}{\sqrt{2}}(\bar{E} \beta, \mathrm{i} \mathfrak{s}, \bar{E}), & \epsilon_{2}^{\mu} & =-\frac{\mathfrak{s}}{\sqrt{2}}(\bar{E} \beta,-\mathrm{i} \mathfrak{s},-\bar{E}), \\
\epsilon_{3}^{\mu} & =\frac{\mathfrak{s e} e^{\mathrm{i} \mathfrak{s} \theta}}{\sqrt{2}}\left(\bar{E} \beta, \bar{E} s_{\theta}+\mathfrak{i} \mathfrak{s} c_{\theta}, \bar{E} c_{\theta}-\mathfrak{i} \mathfrak{s} s_{\theta}\right), & \epsilon_{4}^{\mu} & =-\frac{\mathfrak{s} e^{\mathrm{i} s}}{\sqrt{2}}\left(\bar{E} \beta,-\bar{E} s_{\theta}-\mathfrak{i} \mathfrak{s} c_{\theta},-\bar{E} c_{\theta}+\mathfrak{i} \mathfrak{s} s_{\theta}\right),
\end{aligned}
$$

where we have defined $\bar{E}=E / m$.

For $\widetilde{m}>0$, the propagators for the Abelian and non-Abelian topological gauge theories can be derived as follows:

$$
\begin{aligned}
& \mathcal{D}_{\mu \nu}(p)=-\mathrm{i}\left[\frac{1}{p^{2}+m^{2}}\left(\eta_{\mu \nu}-\frac{p_{\mu} p_{\nu}}{p^{2}}-\frac{\mathrm{i} m \varepsilon_{\mu \nu \rho} p^{\rho}}{p^{2}}\right)+\xi \frac{p_{\mu} p_{\nu}}{p^{4}}\right], \\
& \mathcal{D}_{\mu \nu}^{a b}(p)=\delta^{a b} \mathcal{D}_{\mu \nu}(p) .
\end{aligned}
$$

In eq. (A.11a), the pole $p^{2}=0$ is unphysical, for which the $\operatorname{EOM}(2.5)$ becomes $\widetilde{m} \varepsilon^{\mu \rho \nu} p_{\rho} \epsilon_{\nu}=0$. It can be solved as $\epsilon^{\mu}=f(p) p^{\mu}$, but it can be eliminated by the freedom of gauge transformations [37]. Hence, the massless mode is a pure gauge artifact [37, 65]. Furthermore, we can derive the following Feynman rule of the cubic gauge boson vertex:

$$
V_{\mu \nu \rho}^{a b c}\left(p_{1}, p_{2}, p_{3}\right)=g C^{a b c}\left[\eta_{\mu \nu}\left(p_{1}-p_{2}\right)_{\rho}+\eta_{\nu \rho}\left(p_{2}-p_{3}\right)_{\mu}+\eta_{\rho \mu}\left(p_{3}-p_{1}\right)_{\nu}+\mathrm{i} m \varepsilon_{\mu \nu \rho}\right] .
$$

The quartic gauge boson vertex is similar to that of the 4d QCD.

\section{B General power counting method in $d$-dimensions}

In this appendix, extending the $3 \mathrm{~d}$ power counting method of section 3.2 , we present a general power counting formula for the $d$-dimensional spacetime.

In $d$-dimensions, we derive the mass-dimension of a given $S$-matrix element $\mathbb{S}$ as follows:

$$
D_{\mathbb{S}}=d-\frac{d-2}{2} \mathcal{E}
$$


where $\mathcal{E}$ denotes the total number of external states as before. We see that the general formula (B.1) reduces to $D_{\mathbb{S}}=4-\mathcal{E}$ for $d=4$ and $D_{\mathbb{S}}=3-\frac{1}{2} \mathcal{E}$ for $d=3$, respectively. Then, we can deduce the mass-dimension of all the coupling constants in the $S$-matrix element $\mathbb{S}$ :

$$
D_{C}=\sum_{j} \mathcal{V}_{j}\left(d-d_{j}-\frac{d-2}{2} b_{j}-\frac{d-1}{2} f_{j}\right),
$$

where $\mathcal{V}_{j}$ denotes the number of vertices of type- $j$, and the quantities $\left(d_{j}, b_{j}, f_{j}\right)$ denote the numbers of (partial derivatives, bosonic fields, fermionic fields) in each vertex of type- $j$, respectively. We have the following general relations for each Feynman diagram which contributes to the amplitude $\mathbb{S}$,

$$
L=1+I-\mathcal{V}, \quad \mathcal{V}=\sum_{j} \mathcal{V}_{j}, \quad \sum_{j} \mathcal{V}_{j} b_{j}=2 I_{B}+\mathcal{E}_{B}, \quad \sum_{j} \mathcal{V}_{j} f_{j}=2 I_{F}+\mathcal{E}_{F},
$$

where $L$ denotes the number of loops of a given diagram, $\left(I_{B}, \mathcal{E}_{B}\right)$ denote the numbers of (internal,external) bosonic lines in this diagram, and $\left(I_{F}, \mathcal{E}_{F}\right)$ denote the numbers of (internal,external) fermionic lines in the same diagram. With these, we derive the leading energy dependence of the amplitude $\mathbb{S}$ :

$$
D_{E}=D_{\mathbb{S}}-D_{C}=2(1-\mathcal{V})+(d-2) L+\sum_{j} \mathcal{V}_{j}\left(d_{j}+\frac{1}{2} f_{j}\right)
$$

Then, we consider the $d$-dimensional gauge theories (including Chern-Simons term when allowed). By imposing the relations (3.11), we derive the leading energy dependence of the amplitude $\mathbb{S}$ :

$$
D_{E}=\left(\mathcal{E}_{A_{\mathrm{P}}}-\mathcal{E}_{v}\right)+\left(4-\mathcal{E}-\overline{\mathcal{V}}_{3}\right)+(d-4) L,
$$

where $\mathcal{E}_{A_{\mathrm{P}}}$ is the total number of external states of the physical gauge bosons and $\mathcal{E}_{v}$ denotes number of the external states of gauge bosons $v^{a}=v_{\mu} A^{a \mu}$ with the factor $v^{\mu}=\epsilon_{\mathrm{L}}^{\mu}-\epsilon_{\mathrm{S}}^{\mu}$.

Next, we can apply the power counting formula (B.4) to the topologically massive gravity (TMG) theory. For this, we can derive the leading energy-dependence of a pure graviton scattering amplitude $\mathbb{S}$ in the TMG theory, which corresponds to setting $d_{j}=3$ and $f_{j}=0$ in eq. (B.4):

$$
D_{E}=2 \mathcal{E}_{h_{\mathrm{P}}}+\mathcal{V}_{3}+2+(d-2) L .
$$

where $\mathcal{V}_{3}$ denotes the number of cubic vertices from the CS term in the TMG action. For instance, we can check that for the TMG theory of $d=3$, the above eqs. (B.5) and (B.6) just reduce to the power counting formulas (3.12) and (3.13), which we derived for the $3 \mathrm{~d}$ TMYM and TMG theories in section 3.2.

\section{Dirac spinors in $(2+1) d$ spacetime}

The anti-symmetric and symmetric commutation relations for the gamma matrices in $(2+1)$ d spacetime are given by

$$
\left\{\gamma^{\mu}, \gamma^{\nu}\right\}=2 \eta^{\mu \nu}, \quad\left[\gamma^{\mu}, \gamma^{\nu}\right]=2 \varepsilon^{\mu \nu \alpha} \gamma_{\alpha},
$$


where we can choose the gamma matrices as the Pauli matrices [29]:

$$
\gamma^{0}=\mathrm{i} \sigma_{2}=\left(\begin{array}{cc}
0 & 1 \\
-1 & 0
\end{array}\right), \quad \gamma^{1}=\sigma_{1}=\left(\begin{array}{cc}
0 & 1 \\
1 & 0
\end{array}\right), \quad \gamma^{2}=\sigma_{3}=\left(\begin{array}{cc}
1 & 0 \\
0 & -1
\end{array}\right)
$$

The Dirac equation in the $3 \mathrm{~d}$ spacetime is derived as follows [29, 66]:

$$
\left(\not \partial-m_{f}\right) \psi=0
$$

with $\not \partial=\gamma^{\mu} \partial_{\mu}$. Its solution takes the plane wave form $\psi \sim u(p) e^{-\mathrm{i} p \cdot x}+v(p) e^{\mathrm{i} p \cdot x}$. Thus, the spinors $(u, v)$ satisfy the momentum-space equations:

$$
\left(\not p-\mathrm{i} m_{f}\right) u=0, \quad\left(\not p+\mathrm{i} m_{f}\right) v=0 .
$$

Then, solving eq. (C.4) gives the spinor solutions for particle and anti-particle:

$$
u=\frac{1}{\sqrt{-p_{0}+p_{1}}}\left(\begin{array}{c}
p_{2}+\mathrm{i} m_{f} \\
-p_{0}+p_{1}
\end{array}\right), \quad v=\frac{1}{\sqrt{-p_{0}+p_{1}}}\left(\begin{array}{c}
p_{2}-\mathrm{i} m_{f} \\
-p_{0}+p_{1}
\end{array}\right) .
$$

They obey the following spinor identities:

$$
u \bar{u}=-\not p-\mathrm{i} m_{f}, \quad \bar{u} u=-\mathrm{i} 2 m_{f}, \quad v \bar{v}=-\not p+\mathrm{i} m_{f}, \quad \bar{v} v=\mathrm{i} 2 m_{f},
$$

where $\bar{u}=u^{\dagger} \gamma^{0}$ and $\bar{v}=v^{\dagger} \gamma^{0}$.

\section{Topological scattering amplitudes with matter fields}

In this appendix, we present the full amplitudes of the scattering processes discussed in section 4 . For the notational convenience of the following scattering amplitudes, we have defined the parameters $\beta_{ \pm}=1 \pm \beta$ with $\beta=\sqrt{1-m^{2} / E^{2}}$.

The scattering amplitudes of pair annihilation in the topologically massive scalar QED take the following forms:

$$
\begin{aligned}
\mathcal{T}\left[\phi^{-} \phi^{+} \rightarrow A_{\mathrm{P}} A_{\mathrm{P}}\right] & =-\frac{2 e^{2}\left[1-\bar{E}^{4}+\bar{E}^{2}\left(1+\bar{E}^{2}\right) c_{2 \theta}+\mathrm{i} 2 \bar{E}^{3} s_{2 \theta}\right]}{\left(1-2 \bar{E}^{2}\right)^{2}+4 \bar{E}^{2}\left(1-\bar{E}^{2}\right) c_{\theta}^{2}}, \\
\mathcal{T}\left[\phi^{-} \phi^{+} \rightarrow A_{\mathrm{T}} A_{\mathrm{T}}\right] & =-\frac{2 e^{2}\left(1-2 \bar{E}^{4}+2 \bar{E}^{4} c_{2 \theta}\right)}{\left(1-2 \bar{E}^{2}\right)^{2}+4 \bar{E}^{2}\left(1-\bar{E}^{2}\right) c_{\theta}^{2}} .
\end{aligned}
$$

The Compton scattering amplitudes in the topologically massive scalar QED are given by

$$
\begin{aligned}
\mathcal{T}\left[\phi^{-} A_{\mathrm{P}} \rightarrow \phi^{-} A_{\mathrm{P}}\right]= & \frac{e^{2}}{1-\beta\left(1+\beta_{+}\right)-2 \beta^{2} c_{\theta}}\left\{\left(2-\bar{E}^{2} \beta_{-}^{2}\right) \beta^{2}\right. \\
& \left.-\left[1-\beta\left(1+\beta_{+}\right)+\bar{E}^{2} \beta_{-}^{2}\left(1-2 \beta^{2}\right)\right] c_{\theta}-\mathrm{i} 2 \bar{E} \beta_{-} \beta_{+}(1-2 \beta) s_{\theta}\right\}, \\
\mathcal{T}\left[\phi^{-} A_{\mathrm{T}} \rightarrow \phi^{-} A_{\mathrm{T}}\right]= & \frac{2 e^{2}\left[2 \beta^{2}-\left(1-2 \beta-\beta^{2}\right) c_{\theta}\right]}{1-\beta\left(1+\beta_{+}\right)-2 \beta^{2} c_{\theta}} .
\end{aligned}
$$


The scattering amplitudes of pair annihilation in the topologically massive spinor QED are derived as follows:

$$
\begin{aligned}
\mathcal{T}\left[e^{-} e^{+} \rightarrow A_{\mathrm{P}} A_{\mathrm{P}}\right] & =\frac{2 e^{2} \bar{E}\left[2\left(1-\bar{E}^{2}\right)+2 \bar{E}^{2} c_{2 \theta}+\mathrm{i} \bar{E}\left(1+\bar{E}^{2}\right) s_{2 \theta}\right]}{\left(1-2 \bar{E}^{2}\right)^{2}+4 \bar{E}^{2}\left(1-\bar{E}^{2}\right) c_{\theta}^{2}}, \\
\mathcal{T}\left[e^{-} e^{+} \rightarrow A_{\mathrm{T}} A_{\mathrm{T}}\right] & =\frac{\mathrm{i} 4 e^{2} \bar{E}^{4} s_{2 \theta}}{\left(1-2 \bar{E}^{2}\right)^{2}+4 \bar{E}^{2}\left(1-\bar{E}^{2}\right) c_{\theta}^{2}} .
\end{aligned}
$$

The Compton scattering amplitudes in the topologically massive spinor QED:

$$
\begin{aligned}
\mathcal{T}\left[e^{-} A_{\mathrm{P}} \rightarrow e^{-} A_{\mathrm{P}}\right]= & \frac{\mathrm{i} e^{2} \beta\left(1+\tan \frac{\theta}{2}\right)}{2 \beta_{+}\left[1-\beta\left(1+\beta_{+}\right)-2 \beta^{2} c_{\theta}\right]\left(1+s_{\theta}\right)^{\frac{1}{2}}}\left[1+\bar{E}^{2}-4 \beta \beta_{+}\right. \\
& -\bar{E}^{2} \beta^{2}\left(2-\beta^{2}\right)-4 \beta \beta_{+} c_{\theta}-\left(1+\bar{E}^{2} \beta_{-}^{2} \beta_{+}^{2}\right) c_{2 \theta} \\
& \left.-\mathrm{i} 4 \bar{E} \beta \beta_{-} \beta_{+} s_{\theta}-\mathrm{i} 2 \bar{E} \beta_{-} \beta_{+} s_{2 \theta}\right], \\
\mathcal{T}\left[e^{-} A_{\mathrm{T}} \rightarrow e^{-} A_{\mathrm{T}}\right]= & \frac{\mathrm{i} 2 e^{2} \beta\left(1-2 \beta \beta_{+}-c_{\theta}\right)\left(1+c_{\theta}+s_{\theta}\right)}{\beta_{+}\left[1-\beta\left(1+\beta_{+}\right)-2 \beta^{2} c_{\theta}\right]\left(1+s_{\theta}\right)^{\frac{1}{2}}} .
\end{aligned}
$$

The scattering amplitudes of pair annihilation via the color-singlet channel in the topologically massive QCD are connected to that of the TMQED according to eq. (4.25) in section 4.2.1:

$$
\begin{aligned}
& \mathcal{T}_{\mathrm{PP}}\left[|0\rangle_{q} \rightarrow|0\rangle_{A_{\mathrm{P}}}\right]=\frac{g^{2}}{e^{2}} f(N) \mathcal{T}\left[e^{-} e^{+} \rightarrow A_{\mathrm{P}} A_{\mathrm{P}}\right], \\
& \mathcal{T}_{\mathrm{TT}}\left[|0\rangle_{q} \rightarrow|0\rangle_{A_{\mathrm{T}}}\right]=\frac{g^{2}}{e^{2}} f(N) \mathcal{T}\left[e^{-} e^{+} \rightarrow A_{\mathrm{T}} A_{\mathrm{T}}\right] .
\end{aligned}
$$

where the function $f(N)=\frac{1}{2 \sqrt{2}} \sqrt{\left(N^{2}-1\right) / N}$.

\section{E Graviton propagator and scattering amplitude in TMG}

From the action (5.1) together with the gauge-fixing term (5.8), we derive the quadratic term of the graviton fields:

$$
S_{\mathrm{TMG}}=\int \mathrm{d}^{3} x \frac{1}{2} h^{\mu \nu} \mathcal{D}_{\mu \nu \alpha \beta}^{-1} h^{\alpha \beta},
$$

where the inverse of the graviton propagator $\mathcal{D}_{\mu \nu \alpha \beta}^{-1}$ takes the following form:

$$
\begin{aligned}
\mathcal{D}_{\mu \nu \alpha \beta}^{-1}= & \left(1-\frac{1}{2 \xi}\right) \eta_{\mu \nu} \eta_{\alpha \beta} \partial^{2}-\frac{1}{2}\left(\eta_{\mu \alpha} \eta_{\nu \beta}+\eta_{\mu \beta} \eta_{\nu \alpha}\right) \partial^{2}+\left(\frac{1}{\xi}-1\right)\left(\eta_{\mu \nu} \partial_{\alpha} \partial_{\beta}+\eta_{\alpha \beta} \partial_{\mu} \partial_{\nu}\right) \\
& +\frac{1}{2}\left(1-\frac{1}{\xi}\right)\left(\eta_{\mu \alpha} \partial_{\nu} \partial_{\beta}+\eta_{\mu \beta} \partial_{\nu} \partial_{\alpha}+\eta_{\nu \alpha} \partial_{\mu} \partial_{\beta}+\eta_{\nu \beta} \partial_{\mu} \partial_{\alpha}\right) \\
& +\frac{1}{2 m}\left[\varepsilon_{\mu \rho \alpha}\left(\partial_{\nu} \partial_{\beta} \partial^{\rho}-\eta_{\nu \beta} \partial^{2} \partial^{\rho}\right)+(\mu \leftrightarrow \nu)\right]
\end{aligned}
$$

Then, transforming eq. (E.2) into momentum space and imposing the normalization condition

$$
\mathcal{D}_{\mu \nu \alpha \beta}^{-1} \mathcal{D}^{\alpha \beta \rho \sigma}=\frac{\mathrm{i}}{2}\left(\delta_{\mu}^{\rho} \delta_{\nu}^{\sigma}+\delta_{\mu}^{\sigma} \delta_{\nu}^{\rho}\right)
$$


we can derive the massive graviton propagator as follows:

$$
\mathcal{D}_{\mu \nu \alpha \beta}=\frac{\mathrm{i} \Delta_{\mu \nu \alpha \beta}}{2\left(p^{2}+m^{2}\right)},
$$

where the numerator is given by

$$
\begin{aligned}
\Delta_{\mu \nu \alpha \beta}= & -\eta_{\mu \nu} \eta_{\alpha \beta}-\frac{m^{2}}{p^{2}}\left(2 \eta_{\mu \nu} \eta_{\alpha \beta}-\eta_{\mu \alpha} \eta_{\nu \beta}-\eta_{\mu \beta} \eta_{\nu \alpha}\right)-\frac{1}{p^{2}}\left(\eta_{\mu \nu} p_{\alpha} p_{\beta}+\eta_{\alpha \beta} p_{\mu} p_{\nu}\right) \\
& -\frac{1}{p^{4}} p_{\mu} p_{\nu} p_{\alpha} p_{\beta}+\frac{\xi\left(p^{2}+m^{2}\right)-m^{2}}{p^{4}}\left(\eta_{\mu \alpha} p_{\nu} p_{\beta}+\eta_{\mu \beta} p_{\nu} p_{\alpha}+\eta_{\nu \alpha} p_{\mu} p_{\beta}+\eta_{\nu \beta} p_{\mu} p_{\alpha}\right) \\
& +\frac{\mathrm{i} m p^{\rho}}{2 p^{2}}\left(\varepsilon_{\rho \mu \alpha} \eta_{\nu \beta}+\varepsilon_{\rho \mu \beta} \eta_{\nu \alpha}+\varepsilon_{\rho \nu \beta} \eta_{\mu \alpha}+\varepsilon_{\rho \nu \alpha} \eta_{\mu \beta}\right) \\
& -\frac{\mathrm{i} m p^{\rho}}{2 p^{4}}\left(\varepsilon_{\rho \mu \alpha} p_{\nu} p_{\beta}+\varepsilon_{\rho \mu \beta} p_{\nu} p_{\alpha}+\varepsilon_{\rho \nu \beta} p_{\mu} p_{\alpha}+\varepsilon_{\rho \nu \alpha} p_{\mu} p_{\beta}\right) .
\end{aligned}
$$

Using the notation $P_{\mu \nu}=\eta_{\mu \nu}-\frac{p_{\mu} p_{\nu}}{p^{2}}$, we can further express the propagator (E.4)-(E.5) into the following form:

$$
\begin{aligned}
\mathcal{D}_{\mu \nu \alpha \beta}(p)= & -\frac{\mathrm{i}}{2\left(p^{2}+m^{2}\right)}\left(P_{\mu \alpha} P_{\nu \beta}+P_{\mu \beta} P_{\nu \alpha}-P_{\mu \nu} P_{\alpha \beta}\right) \\
& +\frac{\mathrm{i}}{2 p^{2}}\left(P_{\mu \alpha} P_{\nu \beta}+P_{\mu \beta} P_{\nu \alpha}-2 P_{\mu \nu} P_{\alpha \beta}\right)-\frac{\mathrm{i}}{p^{4}}\left(\eta_{\mu \nu} p_{\alpha} p_{\beta}+\eta_{\alpha \beta} p_{\mu} p_{\nu}\right) \\
& +\frac{\mathrm{i} \xi}{2 p^{4}}\left(\eta_{\mu \alpha} p_{\nu} p_{\beta}+\eta_{\mu \beta} p_{\nu} p_{\alpha}+\eta_{\nu \alpha} p_{\mu} p_{\beta}+\eta_{\nu \beta} p_{\mu} p_{\alpha}\right) \\
& -\frac{m p^{\rho}}{4 p^{2}\left(p^{2}+m^{2}\right)}\left(\varepsilon_{\rho \mu \alpha} P_{\nu \beta}+\varepsilon_{\rho \mu \beta} P_{\nu \alpha}+\varepsilon_{\rho \nu \alpha} P_{\mu \beta}+\varepsilon_{\rho \nu \beta} P_{\mu \alpha}\right) .
\end{aligned}
$$

Under the Landau gauge $\xi=0$, the above propagator reduces to

$$
\begin{aligned}
\mathcal{D}_{\mu \nu \alpha \beta}^{\xi=0}(p)= & -\frac{\mathrm{i}}{2\left(p^{2}+m^{2}\right)}\left(P_{\mu \alpha} P_{\nu \beta}+P_{\mu \beta} P_{\nu \alpha}-P_{\mu \nu} P_{\alpha \beta}\right) \\
& +\frac{\mathrm{i}}{2 p^{2}}\left(P_{\mu \alpha} P_{\nu \beta}+P_{\mu \beta} P_{\nu \alpha}-2 P_{\mu \nu} P_{\alpha \beta}\right) \\
& -\frac{m p^{\rho}}{4 p^{2}\left(p^{2}+m^{2}\right)}\left(\varepsilon_{\rho \mu \alpha} P_{\nu \beta}+\varepsilon_{\rho \mu \beta} P_{\nu \alpha}+\varepsilon_{\rho \nu \alpha} P_{\mu \beta}+\varepsilon_{\rho \nu \beta} P_{\mu \alpha}\right) \\
& -\frac{\mathrm{i}}{p^{4}}\left(\eta_{\mu \nu} p_{\alpha} p_{\beta}+\eta_{\alpha \beta} p_{\mu} p_{\nu}\right) .
\end{aligned}
$$

If the last term above is removed by contracting with a conserved current or on-shell physical graviton polarization, the propagator (E.7) agrees with the result of refs. [1,2].

Next, we note that in section 5 we reconstructed the 4-graviton scattering amplitude in eqs. (5.14)-(5.15) which is expressed in terms of the energy variable $\bar{s}_{0}=s_{0} / \mathrm{m}^{2}$. For the sake of comparison, we further reexpress the 4-graviton amplitude in terms of the Mandelstam variable $\bar{s}=s / m^{2}$, which is connected to $\bar{s}_{0}=s / m^{2}$ via $\bar{s}=\bar{s}_{0}+4$. Thus, from eqs. (5.14)-(5.15), we derive the following equivalent expressions:

$$
\mathcal{M}\left[4 h_{\mathrm{P}}\right]=\frac{\kappa^{2} m^{2}\left(Q_{0}+Q_{2} c_{2 \theta}+Q_{4} c_{4 \theta}+Q_{6} c_{6 \theta}+\bar{Q}_{2} s_{2 \theta}+\bar{Q}_{4} s_{4 \theta}+\bar{Q}_{6} s_{6 \theta}\right) \csc ^{2} \theta}{4096(1-\bar{s}) \bar{s}^{3 / 2}\left[2-\bar{s}-(4-\bar{s}) c_{\theta}\right]\left[2-\bar{s}+(4-\bar{s}) c_{\theta}\right]}
$$


where $\left(Q_{j}, \bar{Q}_{j}\right)$ are expressed as polynomial functions of the variable $\bar{s}=s / m^{2}$,

$$
\begin{aligned}
Q_{0} & =\left(256+49088 \bar{s}-68880 \bar{s}^{2}+25220 \bar{s}^{3}-2768 \bar{s}^{4}\right) \bar{s}^{\frac{1}{2}}, \\
Q_{2} & =\left(-768-45568 \bar{s}+65568 \bar{s}^{2}-19008 \bar{s}^{3}+505 \bar{s}^{4}\right) \bar{s}^{\frac{1}{2}}, \\
Q_{4} & =4\left(192-176 \bar{s}+20 \bar{s}^{2}+635 \bar{s}^{3}+58 \bar{s}^{4}\right) \bar{s}^{\frac{1}{2}}, \\
Q_{6} & =-\left(256+2816 \bar{s}+2912 \bar{s}^{2}+560 \bar{s}^{3}+17 \bar{s}^{4}\right) \bar{s}^{\frac{1}{2}}, \\
\bar{Q}_{2} & =\mathrm{i}\left(1280-256 \bar{s}+21312 \bar{s}^{2}-8960 \bar{s}^{3}+475 \bar{s}^{4}\right) \bar{s}, \\
\bar{Q}_{4} & =\mathrm{i} 4\left(320-544 \bar{s}+676 \bar{s}^{2}+272 \bar{s}^{3}+5 \bar{s}^{4}\right) \bar{s}, \\
\bar{Q}_{6} & =-\mathrm{i}\left(1280+3584 \bar{s}+1568 \bar{s}^{2}+128 \bar{s}^{3}+\bar{s}^{4}\right) \bar{s} .
\end{aligned}
$$

Open Access. This article is distributed under the terms of the Creative Commons Attribution License (CC-BY 4.0), which permits any use, distribution and reproduction in any medium, provided the original author(s) and source are credited.

\section{References}

[1] S. Deser, R. Jackiw and S. Templeton, Three-dimensional massive gauge theories, Phys. Rev. Lett. 48 (1982) 975 [INSPIRE].

[2] S. Deser, R. Jackiw and S. Templeton, Topologically massive gauge theories, Ann.Phys. 140 (1982) 372 [Erratum ibid. 185 (1988) 406] [INSPIRE].

[3] G.V. Dunne, Aspects of Chern-Simons theory, hep-th/9902115 [INSPIRE].

[4] D. Tong, Lectures on the quantum Hall effect, arXiv:1606.06687 [INSPIRE].

[5] S.S. Chern, Complex manifolds without potential theory, second edition, Springer, Berlin Germany (1979).

[6] F. Englert and R. Brout, Broken symmetry and the mass of gauge vector mesons, Phys. Rev. Lett. 13 (1964) 321 [INSPIRE].

[7] P.W. Higgs, Broken symmetries and the masses of gauge bosons, Phys. Rev. Lett. 13 (1964) 508 [INSPIRE].

[8] P.W. Higgs, Broken symmetries, massless particles and gauge fields, Phys. Lett. 12 (1964) 132 [INSPIRE].

[9] G.S. Guralnik, C.R. Hagen and T.W.B. Kibble, Global conservation laws and massless particles, Phys. Rev. Lett.13 (1964) 585 [INSPIRE].

[10] T.W.B. Kibble, Symmetry breaking in non-Abelian gauge theories, Phys. Rev. 155 (1967) 1554 [INSPIRE].

[11] A. Zee, Quantum field theory in a nutshell, second edition, Princeton University Press, Princeton U.S.A. (2010).

[12] H.-J. He, Y.-P. Kuang and C.P. Yuan, Global analysis for probing electroweak symmetry breaking mechanism at high-energy colliders, in the proceedings of the CCAST workshop on physics at TeV energy scale, July 15-26, Beijing, China (1996), hep-ph/9704276 [InSPIRE].

[13] R.S. Chivukula, D.A. Dicus and H.-J. He, Unitarity of compactified five-dimensional Yang-Mills theory, Phys. Lett. B 525 (2002) 175 [hep-ph/0111016] [INSPIRE].

[14] R.S. Chivukula and H.-J. He, Unitarity of deconstructed five-dimensional Yang-Mills theory, Phys. Lett. B 532 (2002) 121 [hep-ph/0201164] [INSPIRE]. 
[15] R.S. Chivukula, D.A. Dicus, H.-J. He and S. Nandi, Unitarity of the higher dimensional standard model, Phys. Lett. B 562 (2003) 109 [hep-ph/0302263] [INSPIRE].

[16] H.-J. He, Higgsless deconstruction without boundary condition, Int. J. Mod. Phys. A 20 (2005) 3362 [hep-ph/0412113] [INSPIRE].

[17] Z. Bern, J.J.M. Carrasco and H. Johansson, New relations for gauge-theory amplitudes, Phys. Rev. D 78 (2008) 085011 [arXiv: 0805.3993] [INSPIRE].

[18] Z. Bern, J.J.M. Carrasco and H. Johansson, Perturbative quantum gravity as a double copy of gauge theory, Phys. Rev. Lett. 105 (2010) 061602 [arXiv:1004.0476] [INSPIRE].

[19] Z. Bern, J.J. Carrasco, M. Chiodaroli, H. Johansson and R. Roiban, The duality between color and kinematics and its applications, arXiv:1909.01358 [INSPIRE].

[20] H. Kawai, D.C. Lewellen and S.H.H. Tye, A relation between tree amplitudes of closed and open strings, Nucl. Phys. B 269 (1986) 1 [InSPIRE].

[21] S.H. Henry Tye and Y. Zhang, Dual identities inside the gluon and the graviton scattering amplitudes, JHEP 06 (2010) 071 [Erratum ibid. 04 (2011) 114] [arXiv:1003.1732] [INSPIRE].

[22] C. de Rham and G. Gabadadze, Generalization of the Fierz-Pauli action, Phys. Rev. D 82 (2010) 044020 [arXiv: 1007.0443] [INSPIRE].

[23] C. de Rham, G. Gabadadze and A.J. Tolley, Resummation of massive gravity, Phys. Rev. Lett. 106 (2011) 231101 [arXiv: 1011.1232] [INSPIRE].

[24] A. Momeni, J. Rumbutis and A.J. Tolley, Massive gravity from double copy, JHEP 12 (2020) 030 [arXiv: 2004.07853] [inSPIRE].

[25] L.A. Johnson, C.R.T. Jones and S. Paranjape, Constraints on a massive double-copy and applications to massive gravity, JHEP 02 (2021) 148 [arXiv:2004.12948] [INSPIRE].

[26] A. Momeni, J. Rumbutis and A.J. Tolley, Kaluza-Klein from colour-kinematics duality for massive fields, arXiv:2012.09711 [INSPIRE].

[27] Y.-F. Hang and H.-J. He, Structure of Kaluza-Klein graviton scattering amplitudes from gravitational equivalence theorem and double-copy, Phys. Rev. D (2002), in press [arXiv: 2106. 04568] [INSPIRE].

[28] Y. Li, Y. F. Hang, H. J. He, and S. He, Scattering amplitudes of Kaluza-Klein strings and extended massive double-copy, to be published in JHEP (2022), arXiv:2111.12042 [INSPIRE].

[29] A. Agarwal, N. Beisert and T. McLoughlin, Scattering in mass-deformed $N \geq 4$ Chern-Simons models, JHEP 06 (2009) 045 [arXiv:0812.3367] [INSPIRE].

[30] T. Bargheer, S. He and T. McLoughlin, New relations for three-dimensional supersymmetric scattering amplitudes, Phys. Rev. Lett. 108 (2012) 231601 [arXiv:1203.0562] [INSPIRE].

[31] Y.-t. Huang and H. Johansson, Equivalent $D=3$ supergravity amplitudes from double copies of three-algebra and two-algebra gauge theories, Phys. Rev. Lett. 110 (2013) 171601 [arXiv: 1210.2255] [INSPIRE].

[32] N. Moynihan, Scattering amplitudes and the double copy in topologically massive theories, JHEP 12 (2020) 163 [arXiv:2006.15957] [INSPIRE].

[33] D.J. Burger, W.T. Emond and N. Moynihan, Anyons and the double copy, arXiv:2103.10416 [INSPIRE].

[34] N. Moynihan, Massive covariant colour-kinematics in 3D, arXiv:2110.02209 [INSPIRE].

[35] B. Binegar, Relativistic field theories in three-dimensions, J. Math. Phys. 23 (1982) 1511 [INSPIRE]. 
[36] R. Jackiw and V.P. Nair, Relativistic wave equations for anyons, Phys. Rev. D 43 (1991) 1933 [INSPIRE].

[37] R.D. Pisarski and S. Rao, Topologically massive chromodynamics in the perturbative regime, Phys. Rev. D 32 (1985) 2081 [inSPIRE].

[38] R. Banerjee, B. Chakraborty and T. Scaria, Polarization vectors, doublet structure and Wigner's little group in planar field theory, Int. J. Mod. Phys. A 16 (2001) 3967 [hep-th/0011011] [INSPIRE].

[39] H.-J. He, Y.-P. Kuang and X. Li, Further investigation on the precise formulation of the equivalence theorem, Phys. Rev. D 49 (1994) 4842 [InSPIRE].

[40] H.-J. He, Y.-P. Kuang and X. Li, On the precise formulation of equivalence theorem, Phys. Rev. Lett. 69 (1992) 2619 [InSPIRE].

[41] H.-J. He and W.B. Kilgore, The Equivalence theorem and its radiative correction-free formulation for all $R(\xi)$ gauges, Phys. Rev. D 55 (1997) 1515 [hep-ph/9609326] [InSPIRE].

[42] J. Ren, Z.-Z. Xianyu and H.-J. He, Higgs gravitational interaction, weak boson scattering, and Higgs inflation in Jordan and Einstein frames, JCAP 06 (2014) 032 [arXiv:1404.4627] [INSPIRE].

[43] Z.-Z. Xianyu, J. Ren and H.-J. He, Gravitational interaction of Higgs boson and weak boson scattering, Phys. Rev. D 88 (2013) 096013 [arXiv: 1305. 0251] [INSPIRE].

[44] F.L. Bezrukov and M. Shaposhnikov, The standard model Higgs boson as the inflaton, Phys. Lett. B 659 (2008) 703 [arXiv:0710.3755] [INSPIRE].

[45] J. Ellis, H.-J. He and Z.-Z. Xianyu, Higgs inflation, reheating and gravitino production in no-scale supersymmetric GUTs, JCAP 08 (2016) 068 [arXiv: 1606.02202] [INSPIRE].

[46] J. Ellis, H.-J. He and Z.-Z. Xianyu, New Higgs Inflation in a no-scale supersymmetric SU(5) GUT, Phys. Rev. D 91 (2015) 021302 [arXiv:1411.5537] [INSPIRE].

[47] H.-J. He and Z.-Z. Xianyu, Extending Higgs inflation with TeV scale new physics, JCAP 10 (2014) 019 [arXiv: 1405.7331] [INSPIRE].

[48] Z.-Z. Xianyu and H.-J. He, Asymptotically safe higgs inflation, JCAP 10 (2014) 083 [arXiv: 1407.6993] [INSPIRE].

[49] S.-F. Ge, H.-J. He, J. Ren and Z.-Z. Xianyu, Realizing dark matter and Higgs inflation in light of LHC diphoton excess, Phys. Lett. B 757 (2016) 480 [arXiv:1602.01801] [InSPIRE].

[50] H.-J. He, J. Ren and W. Yao, Probing new physics of cubic Higgs boson interaction via Higgs pair production at hadron colliders, Phys. Rev. D 93 (2016) 015003 [arXiv:1506.03302] [INSPIRE].

[51] S. Weinberg, Phenomenological Lagrangians, Physica 96A (1979) 327.

[52] H.J. He, Y.P. Kuang and C.P. Yuan, Estimating the sensitivity of LHC to electroweak symmetry breaking: Longitudinal/Goldstone boson equivalence as a criterion, Phys. Rev. D 55 (1997) 3038 [hep-ph/9611316] [INSPIRE].

[53] M. Soldate, Partial wave unitarity and closed string amplitudes, Phys. Lett. B 186 (1987) 321 [INSPIRE].

[54] D.A. Dicus and H.-J. He, Scales of fermion mass generation and electroweak symmetry breaking, Phys. Rev. D 71 (2005) 093009 [hep-ph/0409131] [INSPIRE].

[55] D.A. Dicus and H.-J. He, Scales of mass generation for quarks, leptons and Majorana neutrinos, Phys. Rev. Lett. 94 (2005) 221802 [hep-ph/0502178] [INSPIRE].

[56] E. Witten, Three-dimensional gravity revisited, arXiv:0706.3359 [INSPIRE]. 
[57] E. Witten, (2+1)-dimensional gravity as an exactly soluble system, Nucl. Phys. B 311 (1988) 46 [INSPIRE].

[58] S. Carlip, Quantum gravity in $2+1$ dimensions, Cambridge University Press, Cambridge U.K. (1998).

[59] S. Carlip, Lectures on $(2+1)$ dimensional gravity, J. Korean Phys. Soc. 28 (1995) S447 [gr-qc/9503024] [INSPIRE].

[60] K. Hinterbichler, Theoretical aspects of massive gravity, Rev. Mod. Phys. 84 (2012) 671 [arXiv:1105.3735] [INSPIRE].

[61] R. Sekhar Chivukula, D. Foren, K.A. Mohan, D. Sengupta and E.H. Simmons, Scattering amplitudes of massive spin-2 Kaluza-Klein states grow only as $\mathcal{O}(s)$, Phys. Rev. D 101 (2020) 055013 [arXiv: 1906.11098] [INSPIRE].

[62] R. Sekhar Chivukula, D. Foren, K.A. Mohan, D. Sengupta and E.H. Simmons, Massive spin-2 scattering amplitudes in extra-dimensional theories, Phys. Rev. D 101 (2020) 075013.

[63] J. Bonifacio and K. Hinterbichler, Unitarization from geometry, JHEP 12 (2019) 165 [arXiv: 1910.04767] [INSPIRE].

[64] M. Carrillo-González, A. Momeni and J. Rumbutis, Massive double copy in three spacetime dimensions, JHEP 08 (2021) 116 [arXiv:2107.00611] [INSPIRE].

[65] F.P. Devecchi, M. Fleck, H.O. Girotti, M. Gomes and A.J. da Silva, Canonical quantization of the Maxwell-Chern-Simons theory in the Coulomb gauge, Annals Phys. 242 (1995) 275 [hep-th/9411224] [INSPIRE].

[66] E. Witten, Fermion path integrals and topological phases, Rev. Mod. Phys. 88 (2016) 035001 [arXiv: 1508.04715] [INSPIRE]. 